\title{
Yang-Mills on Surfaces with Boundary: Quantum Theory and Symplectic Limit
}

\author{
Ambar Sengupta \\ Department of Mathematics, Louisiana State University, Baton Rouge, Louisiana 70803-4918, USA. \\ E-mail: sengupta@math.lsu.edu
}

Received: 30 June 1996 / Accepted: 22 July 1996

\begin{abstract}
The quantum field measure for gauge fields over a compact surface with boundary, with holonomy around the boundary components specified, is constructed. Loop expectation values for general loop configurations are computed. For a compact oriented surface with one boundary component, let $\mathcal{M}(\theta)$ be the moduli space of flat connections with boundary holonomy lying in a conjugacy class $\theta$ in the gauge group $G$. We prove that a certain natural closed 2-form on $\mathcal{M}(\theta)$, introduced in an earlier work by $\mathrm{C}$. King and the author, is a symplectic structure on the generic stratum of $\mathcal{M}(\theta)$ for generic $\theta$. We then prove that the quantum Yang-Mills measure, with the boundary holonomy constrained to lie in $\theta$, converges in a natural sense to the corresponding symplectic volume measure in the classical limit. We conclude with a detailed treatment of the case $G=S U(2)$, and determine the symplectic volume of this moduli space.
\end{abstract}

\section{Introduction and Overview of Results}

This paper presents the construction of a quantum gauge field measure over compact surfaces, with specified boundary holonomies, and a determination of the classical limit of this measure when the surface is oriented and has one boundary component.

Results concerming the quantum field measure. The construction of the measure and determination and study of the loop expectation values are carried out in Sects. 1-5. In these sections:

(i) We construct the Euclidean quantum field measure for gauge theory over a compact surface with boundary, with boundary holonomy (or its conjugacy class) specified (the gauge group is a compact connected Lie group).

(ii) Loop expectation values are computed explicitly, and it is shown that they are invariant under appropriate area-preserving surface homeomorphisms. 
The loop expectation value formulas we obtain are quite natural in view of the free-boundary expectation value formulas available in [Fi, Se2,3, Wi1]. Thus one could take them simply as a starting point, rather than a conclusion, from the point of view of lattice gauge theory. However, our objective in this paper has been to derive these expectation value formulas from a continuum theory.

Results concerning the classical limit and sympectic volume. In Sects. 6-11, we focus attention on a compact oriented surface $\Sigma$ of positive genus with one boundary component $\partial \Sigma$. Let $\mathcal{M}(\Theta)$ denote the moduli space of flat connections over $\Sigma$ with holonomy around $\partial \Sigma$ lying in a given conjugacy class $\Theta$ in the gauge group $G$. The gauge group is now taken to be compact connected semisimple. In [KS2] a 2-form $\Omega_{\Theta}$ was defined on the space $\mathcal{A}(\Theta)$ of all connections whose holonomy around $\partial \Sigma$ lie in $\Theta$. It was shown that $\Omega_{\Theta}$ descends naturally to a closed 2-form $\Omega_{\Theta}$ on the moduli space $\mathcal{M}(\Theta)=\mathcal{A}^{0}(\Theta) / \mathcal{G}$, where $\mathcal{A}^{0}(\Theta)$ is the set of flat connections in $\mathcal{A}(\Theta)$ and $\mathcal{G}$ is the group of gauge transformations. In [KS2] it was proven that $\bar{\Omega}_{\Theta}$ is non-degenerate on the 'smooth part' of $\mathcal{M}(\Theta)$ when the conjugacy class $\theta$ passes through a certain neighborhood of the identity in $G$; the proof of non-degeneracy in [KS2] was obtained by 'perturbation' of the case $\Theta=\{e\}$, the latter case being dealt with by means of an earlier result in [KS1]. In the present paper we prove the following results:

(iii) $\bar{\Omega}_{\Theta}$ is non-degenerate on (the 'smooth part' of) $\mathcal{M}(\Theta)$ for generic conjugacy classes $\theta$, i.e. for all $\Theta$ passing through a dense open subset of $G$ - the proof rests on a determinant identity (7.8.1) proven in Lemma 7.8.

(iv) The Yang-Mills quantum-field measure

$$
d \mu_{T}^{\Theta}(\omega)=Z_{T}(\Theta)^{-1} e^{-S_{Y M}(\omega) / T} \delta_{\Theta}(h(C ; \omega))[\mathcal{D} \omega]
$$

converges, as $T \downarrow 0$, to the normalized symplectic volume measure on $\mathcal{M}(\theta)$. A precise statement and the notation will be explained later; the determinant identity (7.8.1) is again the key

(v) the symplectic volume of $\mathcal{M}(\Theta)$, in the case $G=S U(2)$, is computed in Theorem 9.1:

$$
\operatorname{vol}_{\bar{\Omega}_{\theta}}(\mathcal{M}(\Theta))= \begin{cases}2 \pi(\pi-\theta)\left[\frac{\operatorname{vol}(S U(2))}{2 \pi^{2}}\right]^{\frac{2}{3}} & \text { if } g=1 \\ 4 \pi \sin \theta\left[\frac{\operatorname{vol}(S U(2))}{2 \pi^{2}}\right]^{\frac{2}{3}} \operatorname{vol}(S U(2))^{2 g-2} \sum_{n=1}^{\infty} \frac{\chi_{n}(c)}{n^{2 g}-1} & \text { if } g \geq 2\end{cases}
$$

wherein $c$ is any element of $\theta, \chi_{n}(c)=\sin n \theta / \sin \theta$, with $\theta$ specified by $\operatorname{Tr} c=$ $2 \cos \theta$. (The formula for $g \geq 2$ is also valid for $g=1$ )

Related recent works. Recent interest in 2-dimensional quantum gauge theory, attested to by, for instance, the works [AIK, Be, Di, Fi, Fo, Je, KS1-3, RR, Se1-5, Wi1-3], stems in part from questions associated to a 'classical limit' of the quantum theory; in particular, in determining the relationship of the classical limit of the quantum field measure, over oriented surfaces, to a symplectic volume measure on the moduli space of flat connections. The results of Sects. 6-11 of the present paper address the natural extension of this question to the case where we consider connections over surfaces with boundary with the holonomies around the boundary components known up to conjugation. The investigation of the limiting quantum Yang-Mills measure arose in the case of closed surfaces in [Se1] and in Witten's papers [Wi1,2]; a description of some 
of the questions in this area is given in [Wi3]. The work [Fo] of Forman, and [Se4,5] are also devoted to the case of closed oriented surfaces. The most influential early works on the symplectic structure on the moduli space of flat connections on closed oriented surfaces are by Atiyah and Bott [AB] and Goldman [Go]. It seems likely that the symplectic structure that is the subject of the present work is the same as the one obtained through group-cohomological techniques by Biswas and Guruprasad [BG].

In [Wi1], Witten gives formulas for the symplectic volumes of moduli spaces of flat connections over a surface with punctures, with holonomies around these punctures lying in specified conjugacy classes. For a genus $\mathrm{g}$ Riemann surface with one puncture, with the holonomy lying in a conjugacy class $\theta$, the volume given by Witten $((3.18)$ or (4.116) in [Wi1]) is

$$
2 \frac{1}{2^{g-1} \pi^{2 g-1}} \sum_{n=1}^{\infty} \frac{\sin n \theta}{n^{2 g-1}}
$$

where $\theta$ is such that $\operatorname{Tr} a=2 \cos \theta$ for $a \in \Theta$. To compare this with our volume formula given in (v) above (or Theorem 9.1) we need to note that: (i) the metric on $S U(2)$ used in [Wil] is given by $\langle a, b\rangle=-\operatorname{Tr}(a b)$, and (ii) the symplectic form used in [Wi1] is $\frac{1}{4 \pi^{2}}$ times ours (Eq. (2.29) in [Wi1]). This inner-product on $S U(2)$ corresponds to taking $S U(2)$ to be a 3 -sphere of radius $2^{1 / 2}$; its volume then is $2 \pi^{2}\left(2^{1 / 2}\right)^{3}$. Moreover, since Witten's symplectic form is $\left(4 \pi^{2}\right)^{-1}$ times ours, and since $\mathcal{M}(\theta)$ has dimension $6 g-4$, we must multiply our volume formula by $\left(4 \pi^{2}\right)^{-(6 g-4) / 2}$. Putting all these pieces together in the volume formula given in (v) above, we get

$$
\begin{array}{r}
\frac{1}{\left(4 \pi^{2}\right)^{(6 g-4) / 2}} 4 \pi \sin \theta \cdot 2 \cdot\left(2 \pi^{2} 2^{3 / 2}\right)^{2 g-2} \sum_{n=1}^{\infty} \frac{\sin n \theta / \sin \theta}{n^{2 g-1}} \\
=2 \frac{1}{2^{g-1} \pi^{2 g-1}} \sum_{n=1}^{\infty} \frac{\sin n \theta}{n^{2 g-1}}
\end{array}
$$

in pleasant agreement with the expression given by Witten.

\section{Notation and Background for Construction of the Yang-Mills Measure}

2.1 The surface $\Sigma$ as a quotient of the disk $D$. We shall work with a compact 2-dimensional Riemannian manifold $\Sigma$. In Sects. 3 and $4, \Sigma$ is a torus with one hole, i.e. with one boundary component. It will be convenient to view this $\Sigma$ as a quotient of the disk $D=\left\{(x, y) \in \mathbf{R}^{2}: x^{2}+y^{2} \leq 1\right\}$ in the following way. Consider the path $t \mapsto x_{t} \stackrel{\text { def }}{=}(\cos 2 \pi t, \sin 2 \pi t)$ tracing out $\partial D$. If $r<s$ then $x_{r} x_{s}$ will denote the path $t \mapsto x_{t}$ with $t \in[r, s]$. Divide $\partial D$ into arcs $K_{1}, K_{2}, \ldots, K_{7}$, where $K_{j}$ is given by $x_{t}$ with $t \in\left[\frac{j-1}{2 \pi}, \frac{j}{2 \pi}\right], j \in\{1, \ldots, 7\}$. Identify $K_{1}$ with $\bar{K}_{3}$ (the reverse of $K_{3}$ ), $K_{2}$ with $\bar{K}_{4}$, and $K_{5}$ with $\bar{K}_{7}$, linearly. This yields the quotient space $\Sigma$ and the quotient map $q: D \rightarrow \Sigma$. We shall equip $\Sigma$ with the orientation which makes $q$ orientation preserving. The point $o=q(O)$, where $O=(0,0)$ is the center of $D$, will serve as a basepoint on $\Sigma$. The loops

$$
A=q\left(x_{0} O\right) q\left(K_{1}\right) q\left(O x_{0}\right)
$$




$$
\begin{gathered}
B=q\left(x_{0} O\right) q\left(K_{2}\right) q\left(O x_{0}\right), \\
C=q\left(x_{0} O\right) q\left(K_{7} K_{6} K_{5}\right) q\left(O x_{0}\right)
\end{gathered}
$$

generate $\pi_{1}(\Sigma, o)$ subject to the relation

$$
C \bar{B} \bar{A} B A=I
$$

wherein $I$ is the identity in $\pi_{1}(\Sigma, o)$. The presence of $K_{5}$ and $K_{7}$ is not essential; however, when $\Sigma$ is a general compact surface with more than one boundary component (as will be the case in section 5) then arcs like $K_{5}$ and $K_{7}$ must be included, and this is why we choose to keep them in our framework.

2.2 The G-bundle $\pi: P \rightarrow \Sigma$, space of connections $\mathcal{A}$, holonomies $h(\kappa ; \omega)$, and curvature $\Omega^{\omega}$. We shall work with a principal $G$-bundle $\pi: P \rightarrow \Sigma$, where $G$ is a compact connected Lie group with Lie algebra $\underline{g}$ having an Ad-invariant inner-product $\langle\cdot, \cdot\rangle_{g}$ on it. The set of all connections on $P$ will be denoted $\mathcal{A}$. The metrics on $\Sigma$ and on $g$ induce a metric on $\mathcal{A}$ in a standard way. Fix once and for all a basepoint $u \in \pi^{-1}(o)$. If $\kappa:[r, s] \rightarrow \Sigma$ is a path on $\Sigma$ with $\kappa(r)=o$, and if $\omega \in \mathcal{A}$, then we denote by $\tau_{\omega}(\kappa) u$ the parallel translate of $u$ along $\kappa$ with respect to $\omega$. Thus if $\kappa$ is a loop based at $o$ then the holonomy of $\omega$ around $\kappa$, with $u$ as initial point, is $h(\kappa ; \omega) \in G$ given by $\tau_{\omega}(\kappa) u=u h(\kappa ; \omega)$. The curvature of $\omega \in \mathcal{A}$ will be denoted $\Omega^{\omega}$; thus $\Omega^{\omega}=d \omega+\frac{1}{2}[\omega, \omega]$.

2.3 The gauge transformation groups $\mathcal{G}, \mathcal{G}_{0}$, acting on $\mathcal{A}$. We shall use the set $\mathcal{G}$ of automorphisms of $P$, i.e. diffeomorphisms $\phi: P \rightarrow P$ for which $\pi \circ \phi=\pi$ and $\phi(p g)=\phi(p) g$ for all $p \in P, g \in G$. This is a group under composition, and the subgroup $\mathcal{G}_{o} \stackrel{\text { def }}{=}\{\phi \in \mathcal{G}: \phi(u)=u\}$ will be of use. These groups act on $\mathcal{A}$ by $(\phi, \omega) \mapsto \phi^{*} \omega$.

2.4 The Yang-Mills action. If $\omega \in \mathcal{A}$ then the Yang-Mills action $S_{\mathrm{YM}}(\omega)$ is given by

$$
S_{\mathrm{YM}}(\omega)=\frac{1}{2} \int_{\Sigma}\left\|\Omega^{\omega}\right\|_{\underline{g}}^{2} d \sigma,
$$

where $d \sigma$ is the Riemannian surface area measure on $\Sigma$, and $\left\|\Omega^{\omega}\right\|_{\underline{g}}^{2}$ is the function on $\Sigma$ given by

$$
\left\|\Omega^{\omega}\right\|_{\underline{g}}^{2}(x)=\left\|\Omega^{\omega}\left(e_{1}, e_{2}\right)\right\|_{\underline{g}}^{2},
$$

where $x$ runs over $\Sigma$, and $e_{1}, e_{2} \in T_{p} P$, for any $p \in \pi^{-1}(x)$, are such that $\left(\pi_{*} e_{1}, \pi_{*} e_{2}\right)$ is an orthonormal basis of $T_{x} \Sigma$. Since $\Omega^{\omega}$ vanishes when to applied vertical vectors and since it is a 2 -form, it follows from the Ad-invariance of $\langle\cdot, \cdot\rangle_{g}$ that $\left\|\Omega^{w}\right\|_{g}^{2}$ is a well-defined function $\Sigma$. Furthermore, $S_{\mathrm{YM}}$ is invariant under the $\mathcal{G}$-action and therefore defines a function on $\mathcal{A} / \mathcal{G}$ and on $\mathcal{A} / \mathcal{G}_{o}$.

2.5 The curvature function $F^{\omega}$, and the parallel-transport equation. Let $\omega \in \mathcal{A}$. We shall use the map $s_{\omega}: D \rightarrow P$ given by $s_{\omega}(x)=\tau_{\omega}(q(O x)) u$, where $O x$ is the radial path from $O$ to $x$. A convenient way to express the curvature is by means of the map

$$
F^{\omega}: D \rightarrow \underline{g}: x \mapsto F^{\omega}(x) \stackrel{\text { def }}{=} \Omega^{\omega}\left(e_{1}, e_{2}\right),
$$


where on the right $e_{1}, e_{2} \in T_{p} P$, with $p=s_{\omega}(x)$, are such that $\left(\pi_{1} e_{1}, \pi_{*} e_{2}\right)$ is a positively oriented orthonormal basis of $T_{q(x)} \Sigma$. (If $\Sigma$ were an unorientable surface the orientation on $T_{q(x)} \Sigma$ here would be the one which would make $q$ orientation-preserving in a neighborhood of $x$.) Then

$$
S_{\mathrm{YM}}(\omega)=\frac{1}{2} \int_{D}\left\|F^{\omega}\right\|_{\underline{g}}^{2} d \sigma
$$

with $d \sigma$ here being the surface area measure for $\Sigma$ pulled up to $D$ by $q$.

We shall almost always work with admissible curves on $\Sigma$; by an admissible curve we mean a curve of the form $q \circ \kappa$, where $\kappa:[0,1] \rightarrow D: t \mapsto \kappa_{t}$ is a path which can be expressed (i.e. reparametrized) in polar coordinates ' $r=r(\theta)$ ' (thus $\kappa$ cuts every radius, excluding $O$, at most once). If $\kappa$ is such a path and if $q \circ \kappa$ is piecewise smooth, then for each $t \in[0,1]$ we have a loop $q\left(\kappa_{t} O\right) \cdot q \circ \kappa \mid[0, t] \cdot q\left(O \kappa_{0}\right)$; these loops will be very useful. The holonomy

$$
h_{t}(\omega) \stackrel{\text { def }}{=} h\left(q\left(\kappa_{t} O\right) \cdot q \circ \kappa \mid[0, t] \cdot q\left(O \kappa_{0}\right) ; \omega\right)
$$

satisfies the differential equation of parallel-transport:

$$
d h_{t}(\omega) h_{t}(\omega)^{-1}=-d\left(\int_{D^{\kappa_{t}}} F^{\omega} d \sigma\right),
$$

where $D^{\kappa}{ }_{t}$ is the subset of $D$ whose positive boundary is $\kappa_{t} O \cdot \kappa \mid[0, t] \cdot O \kappa_{0}$, i.e. $D_{t}^{\kappa}=\left\{r x_{s}: r \in[0, \kappa(s)], s \in[0, t]\right\}$.

2.6 The map $\omega \mapsto\left(F^{\omega}, h(A ; \omega), h(B ; \omega), h(C ; \omega)\right)$. The map

$$
\omega \mapsto\left(F^{\omega}, h(A ; \omega), h(B ; \omega), h(C ; \omega)\right)
$$

induces a one-to-one map from the quotient space $\mathcal{A} / \mathcal{G}_{o}$. However, the image is constrained by the condition (cf. (2.1.1))

$$
h(C ; \omega) h(B ; \omega)^{-1} h(A ; \omega)^{-1} h(B ; \omega) h(A ; \omega)=h\left(\omega\left(\bar{L}_{0} \cdot \partial D \cdot L_{0}\right) ; \omega\right),
$$

where $L_{0}$ is the radial path from $O$ to $x_{0}=(1,0)$, and $h\left(\omega\left(\bar{L}_{0} \cdot \partial D \cdot L_{0}\right) ; \omega\right)$ is computable, by means of (2.5.4), in terms of only $F^{\omega}$.

2.7 Yang-Mills measures $\mu_{T}, \mu_{T}^{c}, \mu_{T}^{\Theta}$ for the spaces $\mathcal{A}, \mathcal{A}_{c}, \mathcal{A}_{\Theta}$. Let $T>0$. The Yang-Mills measure for $\mathcal{A}$ is, informally, a probability measure $\mu_{T}$ on $\mathcal{A} / \mathcal{G}$ given by the heuristic formula

$$
d \mu_{T}([\omega])=\frac{1}{Z_{T}} e^{-S_{Y M}(\omega) / T}[\mathcal{D} \omega],
$$

where $[\mathcal{D} \omega]$ is the formal Riemannian volume measure on $\mathcal{A}$ pushed down to $\mathcal{A} / \mathcal{G}$, and $Z_{T}$ is a 'normalizing constant'. A rigorous construction of $\mu_{T}$ is given in [Se2].

Let $c \in G$, and consider

$$
\mathcal{A}_{c}=\{\omega \in \mathcal{A}: h(C ; \omega)=c\},
$$

If $\Theta$ is a conjugacy class in $G$, we consider also 


$$
\mathcal{A}_{\Theta}=\{\omega \in \mathcal{A}: h(C ; \omega) \in \Theta\} .
$$

Our goal is to construct a probability measure $\mu_{c}$ on (an appropriate completion of) $\mathcal{A}_{c} / \mathcal{G}_{o}$, and a probability measure $\mu_{\Theta}$ on $\mathcal{A}_{\Theta} / \mathcal{G}_{o}$ which are given heuristically by

$$
\begin{array}{r}
d \mu_{T}^{c}(\omega)=\frac{1}{Z_{T}(c)} \delta\left(h(C ; \omega) c^{-1}\right) e^{-S_{\mathrm{YM}}(\omega) / T}[\mathcal{D} \omega] \text { on } \mathcal{A}_{c} / \mathcal{G}_{o}, \\
d \mu_{T}^{\Theta}(\omega)=\frac{1}{Z_{T}(\Theta)} \delta_{\Theta}(h(C ; \omega)) e^{-S_{\mathrm{YM}}(\omega)}[\mathcal{D} \omega] \text { on } \mathcal{A} / \mathcal{G},
\end{array}
$$

wherein $Z_{T}(c)$ and $Z_{T}(\Theta)$ are "normalizing constants" and $\delta_{\Theta}$ is the $\delta$-function specified by " $\int_{G} f(x) \delta_{\Theta}(x) d x$ " $=\int_{\Theta} f(x) d_{\Theta}(x) \stackrel{\text { def }}{=} \int_{G} f\left(k \theta k^{-1}\right) d k$ for any $\theta \in \Theta$ (and $d k$ is the unit-mass Haar measure on $G$ ).

Actually we will realize these measures on certain larger spaces $\Omega_{c}$ and $\Omega_{\Theta}$.

\section{Construction of the Measures $\mu_{T}^{c}$ and $\mu_{T}^{\Theta}$}

3.1 The Strategy. Since $S_{\mathrm{YM}}(\omega)=\frac{1}{2}\left\|F^{\omega}\right\|_{L^{2}(D ; g)}^{2}$, the expression (2.7.5) for $\mu_{T}^{\Theta}$ suggests that it is reasonable to construct $\mu_{T}^{\Theta}$ as a Gaussian measure on $L^{2}(D ; g)$ times Haar measure on $G^{3}$ (corresponding to the holonomies $h(A ; \omega), h(B ; \omega)$ and $h(C ; \omega)$ ) conditioned to satisfy the constraint (2.6.2) as well as the constraint $h(C ; \omega) \in \Theta$ (recall that $\Theta$ is a conjugacy class in $G)$. Similarly, for $\mu_{T}^{c}$ we must use the constraint $h(C ; \omega)=c$ in addition to (2.6.2).

3.2 Stochastic parallel transport and holonomy over the disk $D$. As is well known, the standard Gaussian measure 'on' $L^{2}(D ; \underline{g})$ actually lives on a Hilbert-Schmidt closure $\overline{L^{2}(D ; g)}$. Henceforth we shall write $\Omega_{\mathrm{disk}}$ for the space $\overline{L^{2}(D ; \underline{g})}$, and $\mu_{\mathrm{T}, \mathrm{disk}}$ for the Gaussian measure, with covariance scaled by $T>0$, on it. The probability space for quantum gauge theory over the disk $D$ is:

$$
\left(\Omega_{\mathrm{disk}}, \mu_{\mathrm{T}, \mathrm{disk}}\right)=\left(\overline{L^{2}(D ; \underline{g})}, \mu_{\mathrm{T}, \mathrm{disk}}\right)
$$

For $\omega \in \Omega_{\text {disk }}, F^{\omega}$ now corresponds to a $\underline{g}$-valued white-noise; i.e. to each Borel set $E \subset \mathbf{R}^{2}$ there is a Gaussian $\underline{g}$-valued random variable $\omega \mapsto \int_{E} F^{\omega} d \sigma \stackrel{\text { def }}{=}\left(F^{\omega}, 1_{E}\right)$; a more detailed account is given in Sect. A3 of the Appendix. In order to impose the constraint (2.6.2) the meaning of the holonomy $h\left(\bar{L}_{0} \cdot \partial D \cdot L_{0} ; \omega\right)$ needs to be understood for $\omega \in \Omega_{\text {disk }}$. More generally we would like to (and will need) give appropriate meaning to the differential equation for parallel-transport (2.5.4).

Thus let $\kappa:[0,1] \rightarrow D$ be an admissible path, and define parallel-transport along $\kappa$ by reinterpreting (2.5.4) as a Stratonovich stochastic differential equation (this idea is due to L. Gross). Thus in place of (2.5.4) we consider the Stratonovich stochastic differential equation

$$
d h_{t}(\omega) \circ h_{t}(\omega)^{-1}=-d\left(\int_{D_{t}^{\kappa}} F^{\omega} d \sigma\right),
$$

with initial condition $h_{0}(\omega)=e$, 
where $D_{t}^{\kappa}$ is, as before, the region $\left\{r x_{s}: 0 \leq r \leq \kappa(s), 0 \leq s \leq t\right\}$.

The solution $h_{t}(\omega)$ of (3.2.1) can be obtained as a (probabilistic) limit of products of the form $\exp \left(\int_{D_{t_{j}}^{\kappa} \backslash D_{t_{j-1}}^{\kappa}} F^{\omega} d \sigma\right)$, where $t_{0}=0<t_{1}<\cdots<t_{N}=t$ and $\max \mid t_{j}-$ $t_{j-1} \mid \rightarrow 0$.

Now $t \mapsto \int_{D_{t}^{\kappa}} F^{\omega} d \sigma$ is, in law, a $\underline{g}$-valued Brownian motion with time clocked by the quadratic variation which, given that $F^{\omega}$ is Gaussian as described before, is simply $T\left|D_{t}^{\kappa}\right|$, where $\left|D_{t}^{\kappa}\right|$ is the area of $D_{t}^{\kappa}$. Thus the solution of (3.2.1) is, in law, simply a Brownian motion on $G$ with time clocked by $T\left|D_{t}^{\kappa}\right|$ instead of by $t$.

In particular, the density of $h_{t}(\cdot)$, with respect to unit-mass Haar measure on $G$, is $Q_{T\left|D_{i}^{\kappa}\right|}(\cdot)$, where $Q_{t}(x)$ is the heat kernel on $G$ normalized to $\int_{G} Q_{t}(x) d x=1$ (here $d x$ is unit-mass Haar measure on $G$ ).

Furthermore, it is proven in [Se2,3] that if $\kappa_{1}$ and $\kappa_{2}$ are admissible loops in $D$ whose interiors do not overlap then $h\left(\kappa_{1} ; \cdot\right)$ and $h\left(\kappa_{2} ; \cdot\right)$ are independent $G$-valued random variables on the Gaussian probability space $\left(\Omega_{\text {disk }}, \mu_{T, \text { disk }}\right)$.

Thus, if $\kappa_{1}, \ldots, \kappa_{n}$ are non-overlapping admissible loops, based at $O$, in $D$ then the joint distribution measure, under $\mu_{\mathrm{T}, \mathrm{disk}}$, of $\left(h\left(\kappa_{1} ; \omega\right), \ldots, h\left(\kappa_{n} ; \omega\right)\right)$, as a random variable in $\omega$ running over $\Omega_{\text {disk }}$, is

$$
Q_{T A_{1}}\left(y_{1}\right) \cdots Q_{T A_{n}}\left(y_{n}\right) d y_{1} \cdots d y_{n}
$$

where $A_{i}$ is the area enclosed by $\kappa_{i}$ and $d y_{i}$ is unit-mass Haar measure on $G$.

3.3 Construction of the measures $\mu_{c}^{T}$ and $\mu_{T}^{\Theta}$. The construction of the conditional probability, satisfying the constraint (2.6.2), requires the technical artifice of dividing $D$ into a 'lower-half' $D_{L}$ and an 'upper-half' $D_{U}$ and working with $h\left(\bar{L}_{0} \cdot \partial D \cdot L_{0} ; \omega\right)=$ $h\left(\partial D_{L} ; \omega\right) h\left(\partial D_{U} ; \omega\right)$. The full technical details of this construction in a general setting are presented in [Se2,3] and so we shall give here only a conceptual account. In particular, we shall not make explicit the technical role played by $D_{L}$ and $D_{U}$. In the Appendix we shall quote the relevant tools from $[\mathrm{Se} 2,3]$ and explain how they apply in the present context.

As explained in the Appendix (Sect. A3), for any $c \in G$ there is a probability measure $\mu_{T}^{c}$ on the space

$$
\Omega_{c} \stackrel{\text { def }}{=} \Omega_{\mathrm{dis} \mathbf{k}} \times G^{2}
$$

which satisifies (cf. the constraint (2.6.2))

$$
h\left(\bar{L}_{0} \cdot \partial D \cdot L_{0} ; \omega\right)=c b^{-1} a^{-1} b a \quad \text { for } \mu_{T}^{c} \text {-a.e. } \omega=\left(\omega_{1}, a, b\right) \in \Omega_{c}
$$

and is, in a natural and precise sense, the conditioning of the probability measure

$$
\mu_{\mathrm{T}, \mathrm{disk}} \times\left(\text { Haar on } G^{2}\right)
$$

to satisfy the constraint (3.3.2).

Analogously, for any conjugacy class $\Theta$ in $G$, there is a probability measure $\mu_{T}^{\Theta}$ on

$$
\Omega_{\Theta} \stackrel{\text { def }}{=} \Omega_{\text {disk }} \times G^{2} \times \Theta
$$

such that

$$
h\left(\bar{L}_{0} \cdot \partial D \cdot L_{0} ; \omega\right)=c b^{-1} a^{-1} b a \text { for } \mu_{T}^{\Theta} \text {-a.e. } \omega=\left(\omega_{1}, a, b, c\right) \in \Omega_{\Theta}
$$


and $\mu_{T}^{\ominus}$ is the conditioning of the probability measure

$$
\mu_{\mathrm{T}, \mathrm{disk}} \times\left(\text { Haar on } G^{2}\right) \times(G \text {-invariant measure on } \Theta)
$$

to satisfy the constraint (3.3.4).

3.4 Expectation values. Consider a measurable function $\phi=\left(\phi_{1}, \ldots, \phi_{n}\right): \Omega_{\text {disk }} \rightarrow G^{n}$ which has a bounded density $\rho_{\phi}$ on $G^{n}$, and consider a bounded measurable function $f$ on $G^{n} \times G^{2}$. Suppose $\phi_{k} \ldots \phi_{1}=h\left(\bar{L}_{0} \cdot \partial D \cdot L_{0} ; \cdot\right)$ for some $k \in\{1, \ldots, n\}$. Then under simple conditions (detailed in Proposition A6 and the discussion following it) on $\phi$ and $\psi:$

$$
\begin{aligned}
& \int_{\Omega_{c}} f\left(\phi\left(\omega_{1}\right), a, b\right) d \mu_{T}^{c}\left(\omega_{1}, a, b\right)= \\
& \frac{1}{N_{T}(c)} \int_{G^{2}} d a d b \int_{G^{n}} d x f(x, a, b) \rho_{\phi}(x) \delta\left(x_{k} \cdots x_{1}\left(c b^{-1} a^{-1} b a\right)^{-1}\right)
\end{aligned}
$$

where $d a$ and $d b$ are the unit-mass Haar measure on $G, d x=d x_{1} \cdots d x_{n}$, and

$$
N_{T}(c)=\int_{G^{2}} Q_{T|\Sigma|}\left(c b^{-1} a^{-1} b a\right) d a d b
$$

with $Q_{t}$ being the heat kernel on $G$, as in Sect. 3.2, and $|\Sigma|$ the area of $\Sigma$. The significance of the 'delta function' $\delta\left(x_{k} \cdots x_{1} \cdots\right)$ in (3.4.1) is simply that one of the variables $x_{j}$ should be replaced by the value which makes the product $x_{k} \cdots x_{1} \cdots$ equal to the identity (and the integration $d x_{j}$ dropped).

The corresponding result for $\Omega_{\Theta}$ is:

$$
\begin{aligned}
& \int_{\Omega_{\Theta}} f\left(\phi\left(\omega_{1}\right), a, b\right) d \mu_{T}^{\Theta}\left(\omega_{1}, a, b, c\right)=\frac{1}{N_{T}(\Theta)} \int_{\Theta} d_{\Theta} c \\
& \int_{G^{2}} d a d b \int_{G^{n}} d x f(x, a, b) \rho_{\phi}(x) \delta\left(x_{k} \cdots x_{1}\left(c b^{-1} a^{-1} b a\right)^{-1}\right)
\end{aligned}
$$

where $d_{\Theta} c$ is the unit-mass, $G$-invariant measure on the conjugacy class $\Theta$ specified by the integration formula

$$
\int_{\Theta} F(c) d_{\Theta} c=\int_{G} F\left(k c k^{-1}\right) d k \text { for any } c \in \Theta
$$

and the normalizer $N_{T}(\Theta)$ is given by:

$$
N_{T}(\Theta)=\int_{G^{2}} Q_{T|\Sigma|}\left(c b^{-1} a^{-1} b a\right) d a d b d_{\Theta} c
$$

Thus $N_{T}(\Theta)=N_{T}(c)$ if $c \in \Theta$.

These results are taken from Proposition 4.5 of [Se2]; for ease of reference, we quote the exact result in the Appendix (Sect. A6).

The expectation value formulas (3.4.1) and (3.4.4) follow from Proposition A6 (quoted from [Se2,3]) in the manner explained in the discussion following A6 in the Appendix. 


\section{Loop Expectation Values}

4.1 Triangulation of $\Sigma$. We shall always work with a certain type of triangulation ('admissible triangulation') $\mathcal{S}$ of $\Sigma$ obtained by means of the quotient map $q: D \rightarrow \Sigma$ from a triangulation $\mathcal{T}$ of $D$. The triangulation $\mathcal{T}$ of $D$ will consist of radii and crossradial segments (i.e. those which can be parametrized in polar coordinates in the form $r=r(\theta)$ ), and will include the origin as a vertex. We will also assume that the $\operatorname{arcs}$ on $\partial D$ corresponding to the loops $A, B$ and $C$ are made up of 1-simplices of the triangulation (this can always be ensured by subdividing the original triangulation). These technical restrictions are to ensure that the parallel-transport equation (3.2.1) is applicable and so that then the relevant holonomies are meaningful.

4.2 $G$-fields and lassos. An assignment $e \mapsto y_{e} \in G$, with $e$ running over the oriented 1 -simplices of a triangulation, satisfying $y_{\bar{e}}=y_{e}^{-1}$ will be called a $G$-field over the triangulation. If $\kappa$ is a path consisting of oriented 1-simplices of the triangulation, $\kappa=b_{j} \cdots b_{1}$, then we define

$$
y(\kappa) \stackrel{\text { def }}{=} y_{b_{j}} \cdots y_{b_{1}} \text {. }
$$

Let $\mathcal{T}$ be a triangulation of $D$ as described in Sect. 4.1, and $\Delta$ a positively oriented 2 -simplex (triangle) in $\mathcal{T}$. We can join an appropriate vertex of $\Delta$ to the origin $O$ by a radial path consisting of 1 -simplexes of $\mathcal{T}$ and thus form a loop $l_{\Delta}$ based at $O$. In this way we form loops $l_{\Delta_{1}}, \ldots, l_{\Delta_{m}}$, one for each positively oriented 2-simplex $\Delta_{j}$ in $\mathcal{T}$. The loops $\Delta_{i}$ will be called the lassos of the triangulation. This can be done, with the $\Delta_{i}$ ordered appropriately, in such a way that the following hold :

(*) For any $G-$ field $y$ over $\mathcal{T}$ :

$$
y\left(l_{\Delta_{m}}\right) \cdots y\left(l_{\Delta_{1}}\right)=y\left(x_{0} O\right) \cdot y(\partial D) \cdot y\left(O x_{0}\right)
$$

where $\partial D$ is taken to start from $x_{0}=(1,0) \in \partial D$. A complete description of the construction of the loops $l_{\Delta_{i}}$ is given in Theorem 3.1, and the discussion preceding it, of [Se2].

(**) To each loop $\kappa$ in $\mathcal{T}$, based at $o$, we can associate a sequence of the lassos $l_{\Delta_{i_{1}}}, l_{\Delta_{i_{2}}} \ldots$ such that $y(\kappa)=y\left(l_{\Delta_{i_{1}}}\right)^{ \pm 1} y\left(l_{\Delta_{i_{2}}}\right)^{ \pm 1} \ldots$ holds for any $G$-field $y$ over $\mathcal{T}$.

These are simple graphical facts and are actually independent of considerations of $G$-fields (Lemma A3 in [Se2]).

4.3 Stochastic holonomy over $\Sigma$, for $\mu_{T}^{c}$ and $\mu_{T}^{\ominus}$. We shall work with loops on $\Sigma$ based at $O$, which consist of oriented 1-simplices of some triangulation $\mathcal{S}$ of $\Sigma$ of the type described in Sect. 4.1. If $\kappa$ is such a loop then there exists a sequence of loops $\kappa_{1}, \ldots, \kappa_{r}$ such that:

(a) each $\kappa_{j}$ is either the projection by $q$ of a loop $\tilde{\kappa}_{j}$ in $D$ or is one of the basic loops $A, B, C$ and their reverses,

(b) for any $G$-field $y$ over $\mathcal{S}, y\left(\kappa_{r}\right) \cdots y\left(\kappa_{1}\right)=y(\kappa)$. 
The existence of such a decomposition is seen by breaking $\kappa$ into suitable segments and lifting these up to $D$ (a detailed account is given in Sect. 5 of [Se2]). Recall that for the loops $\tilde{\kappa}_{j}$ on $D$ the stochastic holonomy $h\left(\tilde{\kappa}_{j} ; \omega\right) \in G$ is meaningful as a random variable in $\omega \in \Omega_{\text {disk }}$.

For $\omega=\left(\omega_{1}, a, b\right) \in \Omega_{c}$, or for $\omega=\left(\omega_{1}, a, b, c\right) \in \Omega_{\Theta}$, we define

$$
h(\kappa ; \omega) \stackrel{\text { def }}{=} h\left(\kappa_{r} ; \omega\right) \cdots h\left(\kappa_{1} ; \omega\right),
$$

where

$$
h\left(\kappa_{j} ; \omega\right)=\left\{\begin{array}{ll}
h\left(\tilde{\kappa}_{j} ; \omega_{1}\right) & \text { if } \tilde{\kappa}_{j} \text { is the loop in } D \text { projecting to } \kappa_{j} \\
a^{ \pm 1} & \text { if } \kappa_{j} \text { is } A \text { or } \bar{A} \\
b^{ \pm 1} & \text { if } \kappa_{j} \text { is } B \text { or } \bar{B} \\
c^{ \pm 1} & \text { if } \kappa_{j} \text { is } C \text { or } \bar{C}
\end{array} .\right.
$$

Then $h(\kappa ; \omega)$ is a well-defined $G$-valued random variable on $\Omega_{c}$ and $\Omega_{\Theta}$ (that $h(\kappa ; \omega)$ is independent of the choice of the $\kappa_{j}$ is proven in Lemma A2 of [Se2]).

4.4 .

Theorem 1. Let $\kappa_{1}, \ldots, \kappa_{n}$ be loops on $\Sigma$, all based at o, composed of oriented 1 -simplices of a triangulation $\mathcal{S}$ of $\Sigma$ of the type described in Sect. 4.1). Let $f$ be a bounded measurable function on $G^{n}$. Consider any $c \in G$. Then

$$
\begin{aligned}
& f\left(h\left(\kappa_{1} ; \omega\right), \ldots, h\left(\kappa_{n} ; \omega\right)\right) d \mu_{T}^{c}(\omega) \\
& =\frac{1}{N_{T}(c)} \int f\left(x\left(\kappa_{1}\right), \ldots, x\left(\kappa_{n}\right)\right) . \\
& . \delta\left(x(C) c^{-1}\right) \prod_{j=1}^{m} Q_{T\left|\Delta_{j}\right|}\left(x\left(\partial \Delta_{j}\right)\right) d x_{e_{1}} \ldots d x_{e_{M}},
\end{aligned}
$$

where $\Delta_{1}, \ldots, \Delta_{m}$ are the positively oriented 2-simplexes of $\mathcal{S},\left|\Delta_{j}\right|$ is the area of $\Delta_{j}$, $e_{1}, \ldots, e_{M}$ and their reverses are the distinct oriented 1-simplices of $\mathcal{S}, Q_{t}(\cdot)$ is the heat kernel on $G$ normalized to $\int_{G} Q_{t}(x) d x=1, d x$ being unit-mass Haar measure on $G$, and

$$
N_{T}(c)=\int_{G^{2}} Q_{T|\Sigma|}\left(c b^{-1} a^{-1} b a\right) d a d b .
$$

The meaning of the $\delta$-function in (4.4.1) is that for some arbitrary bond $e_{j}$ lying on $\partial \Sigma$ and appearing in the loop $C$, the variable $x_{e_{j}}$ should be replaced by the value which makes $x(C)=c$ and $d x_{e_{j}}$ should be dropped from the integration.

Proof. In view of the observation (**) concerning the decomposition of a loop in $D$ in terms of the lassos $l_{\Delta_{i}}$, and in view of the definition of $h(\kappa ; \omega)$ in (4.3.1), we see that each $h\left(\kappa_{i} ; \omega\right)$ is a product of certain of the $h\left(l_{\Delta_{i}} ; \omega\right)^{ \pm 1}$ 's, $h(A ; \omega)^{ \pm 1}, h(B ; \omega)^{ \pm 1}$, and $h(C ; \omega)^{ \pm 1}$ (the latter being $c^{ \pm 1}$ ).

Thus to $f$ we may associate a bounded measurable function $F$ such that

$$
f\left(h\left(\kappa_{1} ; \omega\right), \ldots, h\left(\kappa_{n} ; \omega\right)\right)=F\left(h\left(l_{\Delta_{1}} ; \omega\right), \ldots, h\left(l_{\Delta_{m}} ; \omega\right), h(A ; \omega), h(B ; \omega), h(C ; \omega)\right)
$$

and, more generally,

$$
f\left(x\left(\kappa_{1}\right), \ldots, x\left(\kappa_{n}\right)\right)=F\left(x\left(l_{\Delta_{1}}\right), \ldots, x\left(l_{\Delta_{m}}\right), a, b, c\right)
$$


for any $G$-field $x$ over the triangulation $\mathcal{S}$ (of course, the $c$-term, being constant, could be dropped from $F$ but that would make the form of $F$ dependent on $c$ ).

Recalling the $\mu_{c}$-expectation-value formula (3.4.1) and the $\mu$-distribution of the random variables $h\left(l_{\Delta_{i}} ; \cdot\right)$ given in (3.2.2), we have:

$$
\begin{aligned}
& \int f\left(h\left(\kappa_{1} ; \omega\right), \ldots, h\left(\kappa_{n} ; \omega\right)\right) d \mu_{T}^{c}(\omega) \\
& =\frac{1}{N_{T}(c)} \int_{G^{n} \times G^{2}} F\left(y_{\Delta_{1}}, \ldots, y_{\Delta_{m}}, a, b, c\right) . \\
& \cdot \delta\left(y_{\Delta_{m}} \cdots y_{\Delta_{1}}\left(c b^{-1} a^{-1} b a\right)^{-1}\right) d a d b \prod_{j=1}^{m} Q_{T\left|\Delta_{j}\right|}\left(y_{\Delta_{j}}\right) d y_{\Delta_{j}}
\end{aligned}
$$

where $N_{T}(c)$ is as in (4.4.2) (which is the same as stated earlier in (3.4.2)).

A combinatorial argument (Lemma A1 in [Se2]) shows that if we take the $y_{\Delta_{i}}$ 's and $a, b, c$ such that $y_{\Delta_{m}} \cdots y_{\Delta_{1}}=c b^{-1} a^{-1} b a$ (as is required by the $\delta(\cdot)$ term in (4.4.5)) then it is possible to associate to this data a $G$-field $x$ over $\mathcal{S}$ in such a way that

$$
\begin{gathered}
x\left(l_{\Delta_{i}}\right)=y_{\Delta_{i}} \text { for } i \in\{1, \ldots, m\}, \\
x(A)=a, x(B)=b, x(C)=c .
\end{gathered}
$$

In (4.4.6a) we have tacitly raised $x$ to a $G$-field, also denoted $x$, on the triangulation of $D$ which projects by $q: D \rightarrow \Sigma$ to the triangulation $\mathcal{S}$ of $\Sigma$.

The goal now is to change variables $\left(\left\{y_{\Delta_{i}}\right\}, a, b\right) \mapsto\left\{x_{e_{i}}\right\}$. In order to do this it is necessary to understand how $\prod_{i=1}^{m} d y_{\Delta_{i}} d a d b$ is transformed. It is shown in the proof of Lemma $A 1$ in [Se2] that the $G$-field $x$ can be chosen in the following way:

(i) for a certain collection of bonds $e$, the variables $x_{e}$ are chosen arbitrarily; for the remaining bonds :

(ii) for one bond $e_{A}^{*}$ lying on $q(\partial D)$ and appearing in the loop $A$ (i.e. $e_{A}^{*}$ lies on the part of $A$ on $q(\partial D)), x\left(e_{A}^{*}\right)$ is the product of $a$ with some of the 'previously' assigned values of $x$;

(iii) for one bond $e_{B}^{*}$ lying on $q(\partial D)$ and appearing in the loop $B, x\left(e_{B}^{*}\right)$ is product of $b$ with some of the 'previously' assigned values of $x$;

(iv) for one bond $e_{C}^{*}$ lying on $\partial \Sigma$ and appearing in the loop $C, x\left(e_{C}^{*}\right)$ is product of $c$ with some of the 'previously' assigned values of $x$;

(v) there is one bond $b_{i}$ corresponding to each $\Delta_{i}$ with $x_{b_{i}}$ chosen to be $y_{\Delta_{i}}$ times some of the 'previously' assigned values of $x$.

(A more precise formulation, along with a specification of what 'previously assigned' means, is given in the proof of Lemma A1 of [Se2].)

From (i)-(v), and the translation-invariance of Haar measure, it follows that in the integral on the right side of (4.4.5), we can introduce the variables $\left\{x_{e_{j}}\right\}$ instead of $\left(\left\{y_{\Delta_{i}}\right\}, a, b\right)$, and then the right side of (4.4.5) equals:

$$
\begin{aligned}
& \frac{1}{N_{T}(c)} \int F\left(x\left(\Delta_{1}\right), \ldots, x\left(\Delta_{m}\right), x(A), x(B), c\right) \\
& . \delta\left(x(C) c^{-1}\right) \prod_{j=1}^{m} Q_{T\left|\Delta_{j}\right|}\left(x\left(\partial \Delta_{j}\right)\right) d x_{e_{1}} \cdots d x_{e_{M}}
\end{aligned}
$$


This calls for some explanation: recall from (i) that certain $x_{e_{i}}$ can be chosen arbitrarily, these are integrated over $G$ in (4.4.7) without changing the value of the integral since $\int_{G} d x=1$; the term $\delta\left(y_{\Delta_{m}} \cdots y_{\Delta_{1}}\left(c b^{-1} a^{-1} b a\right)^{-1}\right)$ disappeared since (4.4.6a,b) and (4.2.1) imply that $y_{\Delta_{n}} \cdots y_{\Delta_{1}}\left(c b^{-1} a^{-1} b a\right)^{-1}=e$ is satisfied automatically by our choice of the $x_{e_{i}}$. Finally the new $\delta(\cdot)$-term $\delta\left(x(C) c^{-1}\right)$ appears because, by condition (iii) above, one of the bond variables $x_{e}$, with $e=e_{C}^{*}$ on $\partial \Sigma$ and appearing in $C$, must be chosen in such a way that $x(C)=c$; this says that this $x_{e}$ is not really a 'variable' but is determined by the other $x_{e_{i}}$ (and $c$ ). That the choice of $e_{C}^{*}$ does not affect the value of the integral (4.4.7) follows by a change-of-variables argument (if $e_{C}^{\prime}$ is another bond on $\partial \Sigma$ appearing in $C$ then, given all the other variables $x_{e}$, a new variable $x\left(e_{C}^{\prime}\right)$ may be introduced in place of $x\left(e_{C}^{*}\right)$ by requiring that $x\left(e_{C}^{\prime}\right)$ be such that $x(C)=c$, a relationship that expresses $x\left(e_{C}^{\prime}\right)$ as a product of $x\left(e_{C}^{*}\right)$ with certain of the other bond-variables $\left.x_{e}\right)$.

Now (4.4.4) and (4.4.6b) imply that (4.4.7) is equal to

$$
\frac{1}{N_{T}(c)} \int f\left(x\left(\kappa_{1}\right), \ldots, x\left(\kappa_{n}\right)\right) \delta\left(x(C) c^{-1}\right) \prod_{i=j}^{m} Q_{T\left|\Delta_{j}\right|}\left(x\left(\partial \Delta_{j}\right)\right) d x_{e_{1}} \cdots d x_{e_{M}} .
$$

Thus, recalling that we started our algebraic manipulations with the loop expectationvalue formula (4.4.5), we see that $\int f\left(h\left(\kappa_{1} ; \omega\right), \ldots, h\left(\kappa_{n} ; \omega\right)\right) d \mu_{T}^{c}(\omega)$ is equal to (4.4.8), thereby proving (4.4.1).

4.5 Invariance of loop expectation values for $\mu_{T}^{c}$. Unlike in the case of the "freeboundary" theory developed in [Se2], we cannot expect the loop expectation values

$$
\int f\left(h\left(\kappa_{1} ; \omega\right), \ldots, h\left(\kappa_{n} ; \omega\right)\right) d \mu_{T}^{c}(\omega)
$$

to be invariant under area-preserving transformations of $\Sigma$. This is because the loop $C$ has been selected out as a special loop and so we must consider only area-preserving homeomorphisms of $\Sigma$ which fix $C$.

Thus we wish to show that if $\phi$ is an area-preserving homeomorphism of $\Sigma$ and $\phi \circ C=C$ then

$$
\begin{aligned}
& \int f\left(x\left(\kappa_{1}\right), \ldots, x\left(\kappa_{n}\right)\right) \delta\left(x(C) c^{-1}\right) . \\
& \cdot \prod_{j=1}^{m} Q_{T\left|\Delta_{j}\right|}\left(x\left(\partial \Delta_{j}\right)\right) d x_{e_{1}} \cdots d x_{e_{M}} \\
& =\int_{m^{\prime}} f\left(x\left(\phi \circ \kappa_{1}\right), \ldots, x\left(\phi \circ \kappa_{n}\right)\right) \delta\left(x(C) c^{-1}\right) . \\
& \cdot \prod_{j=1} Q_{T\left|\Delta_{j}^{\prime}\right|}\left(x\left(\partial \Delta_{j}^{\prime}\right)\right) d x_{e_{1}^{\prime}} \cdots d x_{e_{M^{\prime}}^{\prime}},
\end{aligned}
$$

where on the left we are using a triangulation $\mathcal{S}$ while on the right we are using a triangulation $\mathcal{S}^{\prime}$. The loops $\kappa_{i}$ and $C$ are composed of simplices in $\mathcal{S}$, and the loops $\phi \circ \kappa_{i}$ and $C$ are composed of simplices in $\mathcal{S}^{\prime}$. 
First we observe that either side of (4.5.1) is invariant under subdivisions of the triangulation. This is a consequence of the convolution property of the heat kernel $Q_{t}(\cdot)$ :

$$
\int_{G} Q_{t}\left(x y^{-1}\right) Q_{s}(y z) d y=Q_{t+s}(x z),
$$

which we can use to 'collapse' adjacent 2-simplices $\Delta_{1}^{*}$ and $\Delta_{2}^{*}$ of a subdivision of $\mathcal{S}$ :

$$
\int Q_{T\left|\Delta_{\mathrm{i}}\right|}\left(x\left(\partial \Delta_{1}^{*}\right)\right) Q_{T\left|\Delta_{i}\right|}\left(x\left(\partial \Delta_{2}^{*}\right)\right) d x_{b}=Q_{T\left|\Delta_{\bullet}\right|}\left(x\left(\partial \Delta^{*}\right)\right)
$$

wherein $\Delta^{*}$ is the region formed by collapsing $\Delta_{1}^{*}$ and $\Delta_{2}^{*}$ along their common edge $b$. Extra bond-variables which remain after collapsing such common edges can be integrated away (or combined into bond variables for the undivided triangulation) using $\int d x_{b}=1$.

Since the only way area appears in the right of (4.5.2) is through $\left|\Delta^{*}\right|=\left|\Delta_{1}^{*}\right|+\left|\Delta_{2}^{*}\right|$, it follows that the left side of (4.5.1) depends on areas only through the areas of the connected components $\mathcal{R}_{1}, \ldots, \mathcal{R}_{N}$ of $\Sigma \backslash \cup_{i=1}^{n+1} \operatorname{Im}\left(\kappa_{i}\right)$ (here $\operatorname{Im}\left(\kappa_{i}\right)$ is the image of the path $\kappa_{i}$ as a subset of $\Sigma$, and we have set $\kappa_{n+1} \stackrel{\text { def }}{=} C$, for convenience). A more detailed argument is available in Fact 1 of the Appendix in [Se2].

By the 'Hauptvermutung of topology' (Theorem 4.6 in [Br]), $\mathcal{S}$ and $\mathcal{S}^{\prime}$ have subdivisions $\tilde{\mathcal{S}}$ and $\tilde{\mathcal{S}}^{\prime}$ and there is a simplicial isomorphism $\tilde{\phi}: \mathcal{\mathcal { S }} \rightarrow \tilde{\mathcal{S}}^{\prime}$ obtained by an isotopy of $\phi$, preserving the sets $\operatorname{Im}\left(\kappa_{i}\right)$ and $\operatorname{Im}(C)$, such that $\tilde{\phi} \circ C=C$, and $\tilde{\phi} \circ \kappa_{i}$ and $\phi \circ \kappa_{i}$ are the same when taken as sequences of bonds in $\tilde{\mathcal{S}}^{\prime}$. In view of the invariance of the integrals in consideration under subdivisions we may and will assume that $\tilde{\mathcal{S}}=\mathcal{S}$ and $\tilde{\mathcal{S}}^{\prime}=\mathcal{S}^{\prime}$. Thus $\tilde{\phi}$ is a simplicial isomorphism between $\mathcal{S}$ and $\mathcal{S}^{\prime}$.

By simple relabelling of $x_{e}$ as $x_{\tilde{\phi}(e)}$, the left side of (4.5.1) equals:

$$
\begin{gathered}
\int f\left(x\left(\tilde{\phi} \circ \kappa_{1}\right), \ldots, x\left(\tilde{\phi} \circ \kappa_{n}\right)\right) \delta\left(x(\tilde{\phi} \circ C) c^{-1}\right) . \\
\cdot \prod_{j=1}^{m} Q_{T\left|\Delta_{j}\right|}\left(x\left(\partial \tilde{\phi}\left(\Delta_{j}\right)\right)\right) d x_{e_{1}^{\prime}} \cdots d x_{e_{M}^{\prime}}
\end{gathered}
$$

Since $\tilde{\phi} \circ \kappa_{i}$ and $\phi \circ \kappa_{i}$ are the same as sequences of bonds in $\mathcal{S}^{\prime}$, and since $\tilde{\phi} \circ C=C$, relabelling the simplices makes (4.5.3) equal to:

$$
\begin{gathered}
\int f\left(x\left(\phi \circ \kappa_{1}\right), \ldots, x\left(\phi \circ \kappa_{n}\right)\right) \delta\left(x(C) c^{-1}\right) \\
\prod_{j=1}^{m} Q_{T\left|\tilde{\phi}^{-1}\left(\Delta_{j}^{\prime}\right)\right|}\left(x\left(\partial \Delta_{j}^{\prime}\right)\right) d x_{e_{1}^{\prime}} \cdots d x_{e_{M^{\prime}}^{\prime}},
\end{gathered}
$$

(the number $M^{\prime}$ of edges in $\mathcal{S}^{\prime}$ equals the number $M$ because in the present situation $\mathcal{S}$ and $\mathcal{S}^{\prime}$ are isomorphic). Now, as observed earlier (after (4.5.2)), (4.5.4) depends on the areas $\left|\tilde{\phi}^{-1}\left(\Delta_{j}\right)\right|$ only through certain of their sums; specifically, through the areas of the regions $\tilde{\phi}^{-1} \phi\left(\mathcal{R}_{i}\right)$, where, as before, the $\mathcal{R}_{1}, \ldots, \mathcal{R}_{N}$ are the connected components of the complement of $\operatorname{Im}\left(k_{1}\right) \cup \cdots \cup \operatorname{Im}\left(k_{n}\right) \cup \operatorname{Im}(C)$ in $\Sigma$. Since $\tilde{\phi}$ is an isotopy of $\phi$ through maps taking the subset $\operatorname{Im}\left(k_{1}\right) \cup \ldots \cup \operatorname{Im}\left(k_{n}\right) \cup \operatorname{Im}(C)$ always into the same subset, it follows that $\tilde{\phi}^{-1} \phi\left(\mathcal{R}_{i}\right)=\mathcal{R}_{i}$. Therefore, (4.5.4) equals 


$$
\begin{aligned}
& \int f\left(x\left(\phi \circ \kappa_{1}\right), \ldots, x\left(\phi \circ \kappa_{n}\right)\right) \delta\left(x(C) c^{-1}\right) \\
& \prod_{j=1}^{m^{\prime}} Q_{T\left|\Delta_{j}^{\prime}\right|}\left(x\left(\partial \Delta_{j}^{\prime}\right)\right) d x_{e_{1}^{\prime}} \cdots d x_{e_{M^{\prime}}^{\prime}}
\end{aligned}
$$

and this being the right side of (4.5.1), we have proven (4.5.1).

\subsubsection{Observations.}

(1) The above arguments show that the restriction that $\phi$ is the identity on $C$ may be removed if $C$ were to be replaced by $\phi(C)$ on the right side of (4.5.1).

(2) Since the left side of (4.5.1) involves a triangulation $\mathcal{S}$ and the right side involves $\mathcal{S}^{\prime}$, we may set $\phi$ to be the identity and conclude that either side is independent of the specific triangulation used.

(3) The observation (2) is not surprising in view of the fact that the integrals in (4.5.1) are, up to the constant $N_{T}(c)$, the loop expectation-value as given in Theorem 4.4. Nevertheless, the arguments above give an independent and direct proof (one which also does not depend on the triangulations being of the type described in Sect. 4.1).

We turn now to the corresponding results for $\mu_{T}^{\Theta}$. For this we use the notation $d_{\Theta} c$ to denote the $G$-invariant unit-mass measure on a conjugacy class $\Theta$ in $G$; thus

$$
\int_{\Theta} F(c) d_{\Theta} c=\int_{G} F\left(k c k^{-1}\right) d k \text { for any } c \in \Theta .
$$

4.6.

Theorem 2. Let $\kappa_{1}, \ldots, \kappa_{n}$ be loops on $\Sigma$, all based at $o$, composed of oriented 1 -simplices of a good triangulation $\mathcal{S}$ of $\Sigma$ (of the type described in Sect. 4.1). Let $f$ be a bounded measurable function on $G^{n}$. Consider any conjugacy class $\Theta$ in $G$. Then

$$
\begin{aligned}
& \int f\left(h\left(\kappa_{1} ; \omega\right), \ldots, h\left(\kappa_{n} ; \omega\right)\right) d \mu_{T}^{\Theta}(\omega) \\
& =\frac{1}{N_{T}(\Theta)} \int f\left(x\left(\kappa_{1}\right), \ldots, x\left(\kappa_{n}\right)\right) \delta\left(x(C) c^{-1}\right) \\
& \prod_{j=1}^{m} Q_{T\left|\Delta_{j}\right|}\left(x\left(\partial \Delta_{j}\right)\right) d x_{e_{1}} \cdots d x_{e_{M}} d_{\Theta} c
\end{aligned}
$$

where $\Delta_{1}, \ldots, \Delta_{m}$ are the positively oriented 2-simplexes of $\mathcal{S}, e_{1}, \ldots, e_{M}$ and their reverses are the distinct oriented 1-simplices of $\mathcal{S}, Q_{t}(\cdot)$ is the heat kernel on $G$ normalized to $\int_{G} Q_{t}(x) d x=1, d x$ being unit-mass Haar measure on $G$, and

$$
N_{T}(\Theta)=\int_{G^{2}} Q_{T|\Sigma|}\left(c b^{-1} a^{-1} b a\right) d a d b d_{\Theta} c .
$$

The meaning of the $\delta$-function in (4.6.1) is that for some arbitrary bond $e_{j}$ lying on $\partial \Sigma$ and appearing in the loop $C$, the variable $x_{e_{j}}$ should be replaced by the value which makes $x(C)=c$ and $d x_{e_{j}}$ should be dropped from the integration. 
Proof. The proof is virtually identical to that of Theorem 4.4, except that we use the $\mu_{T}^{\ominus}$ expectation-value formula (3.4.3) as starting point instead of the $\mu_{T}^{c}$-formula (3.4.1) used for proving Theorem 4.4.

\section{7.}

Theorem 3. The loop expectation values $\int f\left(h\left(\kappa_{1} ; \omega\right), \ldots, h\left(\kappa_{n} ; \omega\right)\right) d \mu_{T}^{\Theta}(\omega)$ remain invariant if each $\kappa_{i}$ is replaced by $\phi \circ \kappa_{i}$, where $\phi$ is any area-preserving homeomorphism of $\Sigma$ which preserves the orientation of $C$.

Proof. Recall, from Observation (1) in Sect. 4.5, that the general form of (4.5.3) is:

$$
\begin{aligned}
& \int f\left(x\left(\kappa_{1}\right), \ldots, x\left(\kappa_{n}\right)\right) \delta\left(x(C) c^{-1}\right) \prod_{i=1}^{m} Q_{T\left|\Delta_{j}\right|}\left(x\left(\partial \Delta_{j}\right)\right) d x_{e_{1}} \cdots d x_{e_{M}} \\
& =\int f\left(x\left(\phi \circ \kappa_{1}\right), \ldots, x\left(\phi \circ \kappa_{n}\right)\right) \delta\left(x(\phi \circ C) c^{-1}\right) . \\
& \cdot \prod_{j=1}^{m^{\prime}} Q_{T\left|\Delta_{j}^{\prime}\right|}\left(x\left(\partial \Delta_{j}^{\prime}\right)\right) d x_{e_{1}^{\prime}} \cdots d x_{e_{M^{\prime}}^{\prime}},
\end{aligned}
$$

the notation being as for (4.5.1), and $c$ any element of $G$. We will show that either integral depends not on the full curve $C$ but only on the part of $C$ which lies on $\partial \Sigma$.

In (4.7.1) we can take $c \in \Theta$ and integrate by the $G$-conjugation-invariant measure $d_{\Theta} c$; this yields a formula of the form:

$$
\int[\cdots] \delta\left(x(C) c^{-1}\right)[\cdots] d_{\Theta} c=\int[\cdots]^{\prime} \delta\left(x(\phi \circ C) c^{-1}\right)[\cdots]^{\prime} d_{\Theta} c .
$$

Now the loop $C$ can be expressed as

$$
C=\bar{L} \cdot C_{\partial} \cdot L,
$$

where $C_{\partial}$ lies entirely on $\partial \Sigma$, and $L$ is a path from $o$ to a point on $\partial \Sigma$ where $C$ 'first hits' $\partial \Sigma$. Then

$$
x(C)=x(L)^{-1} x\left(C_{\partial}\right) x(L) .
$$

We substitute this into (4.7.2) and observe that the $\delta(\cdots)$ term on the left can be reordered into the form

$$
\delta\left(x\left(C_{\partial}\right) x(L) c^{-1} x(L)^{-1}\right) .
$$

Now since $d_{\Theta} c$ is a $G$-conjugation-invariant measure on $\Theta$ it follows that in the integrations in (4.7.2), the $\delta$-term (4.7.5a) can be replaced by

$$
\delta\left(x\left(C_{\partial}\right) c^{-1}\right) .
$$

Returning to (4.7.2), we then have

$$
\int[\cdots] \delta\left(x\left(C_{\partial}\right) c^{-1}\right)[\cdots] d_{\Theta} c=\int[\cdots]^{\prime} \delta\left(x\left(\phi \circ C_{\partial}\right) c^{-1}\right)[\cdots]^{\prime} d_{\Theta} c .
$$

Now $C_{\partial}$ and $\phi \circ C_{\partial}$ can differ only in their starting points and orientation. We have assumed that $\phi \circ C$ and $C$ have the same orientation; thus the loops $C_{\partial}$ and $\phi \circ C_{\partial}$ 
can only differ in their starting points. But then $x\left(\phi \circ C_{\partial}\right)$, as a product of the bondvariables $x_{e}$, is a cyclic permutations of $x\left(C_{\partial}\right)$, i.e. a conjugate of $x\left(C_{\partial}\right)$. Since $d_{\Theta} c$ is $G$-conjugation-invariant it follows that in the right side of (4.7.6) $\phi \circ C_{\partial}$ can be replaced by $C_{\partial}$. Tracing our arguments back to (4.7.1) we conclude that:

$$
\begin{aligned}
& \left.x(C) c^{-1}\right) \prod_{j=1}^{m} Q_{T\left|\Delta_{j}\right|}\left(x\left(\partial \Delta_{j}\right)\right) d x_{e_{1}} \cdots d x_{e_{M}} \\
& =\int f\left(x\left(\phi \circ \kappa_{1}\right), \ldots, x\left(\phi \circ \kappa_{n}\right)\right) \delta\left(x(C) c^{-1}\right) \\
& \prod_{j=1}^{m^{\prime}} Q_{T\left|\Delta_{j}^{\prime}\right|}\left(x\left(\partial \Delta_{j}^{\prime}\right)\right) d x_{e_{1}^{\prime}} \cdots d x_{e_{M^{\prime}}^{\prime}},
\end{aligned}
$$

and this what we wished to prove. Note that, as in Sect. 4.5 , taking $\phi$ to be the identity map in (4.7.7) shows that the loop-expectation-value formulas are actually independent of the particular triangulation used.

\section{Other Surfaces}

In this section we will sketch the construction of the boundary-holonomy-restricted quantum gauge field measures for a general compact 2-dimensional Riemannian manifold with boundary, and write down the loop expectation-value formulas.

5.1 Hypotheses on $\Sigma$, and generators of $\pi_{1}(\Sigma, o)$. In this section, $\Sigma$ is a compact 2-dimensional Riemannian manifold with boundary.

Instead of the loops $A, B, C$ of the earlier sections, we now have the following generators of $\pi_{1}(\Sigma, o)$ :

( $\Sigma 1)$ If $\Sigma$ is orientable, has genus $\mathrm{g} \geq 0$, and has $p$ boundary-components, then we have loops

$$
A_{1}, B_{1}, \ldots, A_{\mathrm{g}}, B_{\mathrm{g}}, C_{1}, \ldots, C_{p}
$$

generating $\pi_{1}(\Sigma, o)$ subject to the relation that

$$
C_{p} \cdots C_{1} \bar{B}_{\mathrm{g}} \bar{A}_{\mathrm{g}} B_{\mathrm{g}} A_{\mathrm{g}} \cdots \bar{B}_{1} \bar{A}_{1} B_{1} A_{1} \text { gives the identity in } \pi_{1}(\Sigma, o)
$$

The loops $C_{i}$ are of the form $\bar{L}_{i} C_{i}^{\prime} L_{i}$, where $C_{i}^{\prime}$ traces a loop around boundary component \#i and $L_{i}$ is a simple path from o to a point of $C_{i}^{\prime}$ (the point where $C_{i}$ 'first hits' $\partial \Sigma$ )

( $\Sigma 2)$ If $\Sigma$ is unorientable and has $p$ boundary-components, then we have loops

$$
A_{1}, \ldots, A_{\mathrm{g}}, C_{1}, \ldots, C_{p}
$$

generating $\pi_{1}(\Sigma, o)$ subject to the relation that

$$
C_{p} \cdots C_{1} A_{\mathrm{g}} A_{\mathrm{g}} \cdots A_{1} A_{1} \text { gives the identity in } \pi_{1}(\Sigma, o) .
$$

The loops $C_{i}$ are of the form $\bar{L}_{i} C_{i}^{\prime} L_{i}$, as described for $(\Sigma 1)$. 
5.2 The connection spaces $\mathcal{A}(\theta)$ and $\mathcal{A}(c)$. We are interested in connections $\omega$ with specified values, or restrictions, on the values of the holonomies $h\left(C_{i} ; \omega\right)$. For simplicity we shall work with simultaneous restrictions on all of the $C_{i}$; restrictions on only some of the $C_{i}$ can be handled similarly. Thus we consider the spaces of connections:

$$
\begin{aligned}
& \mathcal{A}\left(c_{1}, \ldots, c_{p}\right) \stackrel{\text { def }}{=}\left\{\omega \in \mathcal{A}: h\left(C_{1} ; \omega\right)=c_{1}, \ldots, h\left(C_{p} ; \omega\right)=c_{p}\right\}, \\
& \text { for any }\left(c_{1}, \ldots, c_{p}\right) \in G^{p}, \\
& \mathcal{A}(\theta) \stackrel{\text { def }}{=}\left\{\omega \in \mathcal{A}:\left(h\left(C_{1} ; \omega\right), \ldots, h\left(C_{p} ; \omega\right)\right) \in \Theta\right\}, \\
& \quad \text { for any } G \text {-conjugation orbit } \theta \text { in } G^{p} .
\end{aligned}
$$

Here, by $G$-conjugation orbit we mean an orbit of the conjugation action of $G$ on $G^{p}$, i.e. a subset of the form $\left\{\left(x c_{1} x^{-1}, \ldots, x c_{p} x^{-1}\right): x \in G\right\}$.

We shall use the notation

$$
\underline{c} \stackrel{\text { def }}{=}\left(c_{1}, \ldots, c_{p}\right) \text {. }
$$

5.3 The measures $\mu_{T}^{\frac{c}{T}}$ and $\mu_{T}^{\ominus}$. Let $T$ be a fixed positive real number. On $\mathcal{A}(c) / \mathcal{G}_{o}$ we are interested in the measure

$$
d \mu_{T}^{c}([\omega])=\frac{1}{Z_{T}(c)} e^{-S_{\mathrm{YM}}(\omega) / T} \delta\left(\left(h\left(C_{1} ; \omega\right), \ldots, h\left(C_{p} ; \omega\right)\right) \underline{c}^{-1}\right)[\mathcal{D} \omega]
$$

while on $\mathcal{A}(\Theta) / \mathcal{G}$ we are interested in the measure

$$
d \mu_{T}^{\Theta}([\omega])=\frac{1}{Z_{T}(\Theta)} e^{-S_{\mathrm{YM}}(\omega) / T} \delta_{\Theta}\left(\left(h\left(C_{1} ; \omega\right), \ldots, h\left(C_{p} ; \omega\right)\right)\right)[\mathcal{D} \omega],
$$

wherein $\delta_{\Theta}$ is specified in the manner explained after (2.7.5).

As in the case of the torus, we start with the Gaussian probability space

$$
\left(\Omega_{\text {disk }}, \mu_{\mathrm{T}, \text { disk }}\right) \text { for the disk } D \text {. }
$$

Then we define

$$
\Omega_{\underline{c}} \stackrel{\text { def }}{=} \begin{cases}\Omega_{\text {disk }} \times G^{2 g} & \text { if } \Sigma \text { is orientable, i.e. satisfies }(\Sigma 1) \\ \Omega_{\text {disk }} \times G^{\mathrm{g}} & \text { if } \Sigma \text { satisifies }(\Sigma 2)\end{cases}
$$

Similarly,

$$
\Omega_{\Theta} \stackrel{\text { def }}{=}\left\{\begin{array}{ll}
\Omega_{\text {disk }} \times G^{2 \mathrm{~g}} \times \Theta & \text { if } \Sigma \text { satisfies }(\Sigma 1) \\
\Omega_{\mathrm{disk}} \times G^{\mathrm{g}} \times \Theta & \text { if } \Sigma \text { satisifies }(\Sigma 2)
\end{array} .\right.
$$

We define the measure $\mu_{c}$ to be $\mu_{\mathrm{T} \text {,disk }} \times$ (Haar on $G^{\sigma \mathrm{g}}$ ) (the $\sigma$ in the exponent being 2 if $(\Sigma 1)$ applies and 1 if $(\Sigma 2)$ applies) conditioned to satisfy the following constraint (recall that $L_{0}$ is the radial path from $O$ to $x_{0}=(1,0) \in \partial D$ ):

$$
h\left(\bar{L}_{0} \cdot \partial D \cdot L_{0} ; \omega_{1}\right)= \begin{cases}c_{p} \cdots c_{1} b_{\mathrm{g}}^{-1} a_{\mathrm{g}}^{-1} b_{\mathrm{g}} a_{\mathrm{g}} \cdots b_{1}^{-1} a_{1}^{-1} b a & \text { if }(\Sigma 1) \text { applies } \\ c_{p} \cdots c_{1} a_{\mathrm{g}}^{2} \cdots a_{1}^{2} & \text { if }(\Sigma 2) \text { applies }\end{cases}
$$

where 


$$
\omega \stackrel{\text { def }}{=}\left\{\begin{array}{ll}
\left(\omega_{1}, a_{1}, b_{1}, \ldots, a_{\mathrm{g}}, b_{\mathrm{g}}\right) & \text { if }(\Sigma 1) \text { applies } \\
\left(\omega_{1}, a_{1}, \ldots, a_{\mathrm{g}}\right) & \text { if }(\Sigma 2) \text { applies }
\end{array} .\right.
$$

Thus the constraint will hold $\mu_{T}^{\frac{c}{T}}$-almost-everywhere.

To define $\mu_{T}^{\Theta}$ we use the same constraint condition on the measure

$$
\mu_{\mathrm{T}, \text { disk }} \times\left(\text { Haar on } G^{\sigma \mathrm{g}}\right) \times(G \text {-invariant unit-mass on } \Theta)
$$

wherein $\sigma$ is as before. The space on which $\mu_{T}^{\ominus}$ will sometimes be denoted $\overline{\mathcal{A}(\Theta) / \mathcal{G}}$.

Expectation values with respect to $\mu_{T}^{\frac{c}{\tau}}$ and $\mu_{T}^{\Theta}$ may be obtained in a way exactly analogous to that explained in Sect. 3.4 for the one-holed torus.

Stochastic holonomies with respect to $\mu_{T}^{\frac{c}{T}}$ and $\mu_{T}^{\Theta}$ are also defined exactly analogously to (4.3.1) in Sect. 4.3.

Carrying out essentially the same arguments as in Sect. 4 we obtain the following generalized version of Theorems 4.4. and 4.6.

5.4 .

Theorem 4. Let $\kappa_{1}, \ldots, \kappa_{n}$ be loops on $\Sigma$, all based at the point $o$, composed of oriented 1 -simplices of a good triangulation $\mathcal{S}$ of $\Sigma$ ( of the type described in Sect. 4.1). Let $f$ be a bounded measurable function on $G^{n}$. Consider any $\underline{c}=\left(c_{1}, \ldots, c_{p}\right) \in G^{p}$. Then

$$
\begin{aligned}
& \int f\left(h\left(\kappa_{1} ; \omega\right), \ldots, h\left(\kappa_{n} ; \omega\right)\right) d \mu_{T}^{c}(\omega) \\
& =\frac{1}{N_{T}(\underline{c})} \int f\left(x\left(\kappa_{1}\right), \ldots, x\left(\kappa_{m}\right)\right) \prod_{i=1}^{p} \delta\left(x\left(C_{i}\right) c_{i}^{-1}\right) \\
& \prod_{j=1}^{m} Q_{T\left|\Delta_{j}\right|}\left(x\left(\partial \Delta_{j}\right)\right) d x_{e_{1}} \cdots d x_{e_{M}},
\end{aligned}
$$

where $\Delta_{1}, \ldots, \Delta_{m}$ are the positively oriented 2 -simplices of $\mathcal{S}, e_{1}, \ldots, e_{M}$ and their reverses are the distinct oriented 1-simplices of $\mathcal{S}, Q_{t}(\cdot)$ is the heat kernel on $G$ normalized to $\int_{G} Q_{t}(x) d x=1, d x$ being unit-mass Haar measure on $G$, and

$$
\begin{aligned}
& N_{T}(\boldsymbol{c}) \\
& \stackrel{\operatorname{def}}{=}\left\{\begin{array}{ll}
\int_{G^{2 \mathrm{~g}}} Q_{T|\Sigma|}\left(c_{p} \cdots c_{1} b_{\mathrm{g}}^{-1} a_{\mathrm{g}}^{-1} b_{\mathrm{g}} a_{\mathrm{g}} \cdots b_{1}^{-1} a_{1}^{-1} b_{1} a_{1}\right) d a_{1} \cdots d b_{\mathrm{g}} & \text { if }(\Sigma 1) \text { applies } \\
\int_{G^{\mathrm{g}}} Q_{T|\Sigma|}\left(c_{p} \cdots c_{1} a_{\mathrm{g}}^{2} \cdots a_{1}^{2}\right) d a_{1} \cdots d a_{\mathrm{g}} & \text { if }(\Sigma 2) \text { applies }
\end{array} .\right.
\end{aligned}
$$

The meaning of the $\delta$ functions in (5.4a) is that, for each $C_{i}$, for some arbitrary bond $e_{j}$ lying on $\partial \Sigma$ and appearing in the loop $C_{i}$, the variable $x_{e_{j}}$ should be replaced by the value which makes $x\left(C_{i}\right)=c_{i}$ and $d x_{e_{j}}$ should be dropped from the integration.

For $\mu_{T}^{\Theta}$, we have:

$$
\begin{aligned}
& \int f\left(h\left(\kappa_{1} ; \omega\right), \ldots, h\left(\kappa_{n} ; \omega\right)\right) d \mu_{T}^{\Theta}(\omega) \\
& =\frac{1}{N_{T}(\Theta)} \int f\left(x\left(\kappa_{1}\right), \ldots, x\left(\kappa_{n}\right)\right) \prod_{i=1}^{p} \delta_{\Theta}\left(x\left(C_{i}\right) c_{i}^{-1}\right) d_{\Theta} c_{i} \\
& \prod_{j=1}^{m} Q_{T\left|\Delta_{j}\right|}\left(x\left(\partial \Delta_{j}\right)\right) d x_{e_{1}} \cdots d x_{e_{M}} .
\end{aligned}
$$

The meaning of the delta-function $\delta_{\Theta}$ and the integrator $d_{\Theta} c$ are explained in Sect. 2.7. The normalizer $N_{T}(\Theta)$ is $\int_{G^{p}} N_{T}(c) d_{\Theta} \underline{c}$. 
The normalizer $N_{T}(c)$ has appeared in the appendix of [Se2] as a topologicallyinvariant function associated to the system of loops $C_{1}, \ldots, C_{p}$ on $\Sigma$. The functions $N_{T}(\Theta)$ have appeared in [Wi1].

As in Sect. 4, the loop expectation value formulas $(5.4 a, b)$ are invariant under area-preserving homeomorphisms of $\Sigma$ which fix the loops $C_{i}$ pointwise. However, for $p>1$, the analog of Theorem 4.7 need not hold.

\section{Spaces of Connections and 2-Forms on Them}

In this section we describe the surface, spaces of connections, and 2-forms which we shall be working with in Sects. 6-9. Some of the notation and definitions are lifted directly from $[\mathrm{KS} 2,3]$.

6.1 The surface $\Sigma$, group $G$, the connection spaces $\mathcal{A}, \mathcal{A}(\Theta), \mathcal{M}(\Theta)$. Henceforth, $\Sigma$ will denote a compact connected oriented 2-dimensional manifold of genus $\mathrm{g} \geq 1$, with a connected non-empty boundary $\partial \Sigma$. In Sects. 8 and $9, \Sigma$ will be taken to be also a Riemannian manifold. We shall use a fixed basepoint $o$ in $\Sigma$, and piecewise smooth loops $A_{1}, B_{1}, \ldots, A_{\mathrm{g}}, B_{\mathrm{g}}, C:[0,1] \rightarrow \Sigma$, all based at a point $o \in \Sigma$, which generate the fundamental group $\pi_{1}(\Sigma, o)$, subject to the relation that $C \bar{B}_{\mathrm{g}} \bar{A}_{\mathrm{g}} B_{\mathrm{g}} A_{\mathrm{g}} \ldots \ldots \bar{B}_{1} \bar{A}_{1} B_{1} A_{1}$ is homotopic to the constant loop at $o$, wherein we denote by $\bar{X}$ the reverse of any path $X$. The loop $C$ is of the form $\vec{L} C^{*} L$, where $C^{*}$ is a simple loop around the boundary $\partial \Sigma$ and $L$ is a path from $o$ to the initial point of $C^{*}$; the loops $A_{i}$ and $B_{i}$ are also of the form $A_{i}=\bar{L} A_{i}^{*} L$ and $B_{i}=\bar{L} B_{i}^{*} L$. A detailed description of these loops is given in [KS3: Sect. 6.1] (and in a wider setting in [Se2]), but we shall not need such details here.

We work with a principal $G$-bundle $\pi: P \rightarrow \Sigma$, where the gauge group $G$ is now assumed to be compact, connected, and semisimple. The semisimplicity hypothesis is not of essential significance but we impose it to focus on the more significant issues.

Since $\Sigma$ has boundary, the bundle $P$ is trivial and so there is a smooth section $s: \Sigma \rightarrow P$. Connections and other forms on $P$ can be pulled down to $\Sigma$ by using $s$. In this way we can and will identify the space $\mathcal{A}$ of all connections on $P$ with the space of all smooth $\underline{g}$-valued 1-forms over $\Sigma$; thus we take

$$
\mathcal{A}=\text { space of all smooth } \underline{g} \text {-valued 1-forms on } \Sigma .
$$

The section $s$ is used only for convenience and the constructions (such as symplectic forms) and results we discuss are independent of the choice of $s$.

A connection $\omega \in \mathcal{A}$ is flat if its curvature is zero, i.e. if $d \omega+\frac{1}{2}[\omega, \omega]=0$. We will be interested in the set of all flat connections :

$$
\mathcal{A}^{0} \stackrel{\text { def }}{=}\{\omega: \omega \in \mathcal{A} \text { and } \omega \text { is flat }\} .
$$

We work with a fixed basepoint $u \in \pi^{-1}(o)$, where $o$ is the basepoint on $\Sigma$. If $\kappa$ is a piecewise smooth loop on $\Sigma$ based at $o$, then, as explained in Sect. 2.2, $h(\kappa ; \omega)$ denotes the holonomy of $\omega$ around $\kappa$, with $u$ as initial point.

We shall denote by $\Theta$ a conjugacy class in the group $G$; we shall work with the spaces

$$
\mathcal{A}(\Theta)=\{\omega \in \mathcal{A}: h(C ; \omega) \in \Theta\} \text { and } \mathcal{A}^{0}(\Theta)=\left\{\omega \in \mathcal{A}^{0}: h(C ; \omega) \in \Theta\right\} .
$$


The group $\mathcal{G}$ (Sect. 2.3) of automorphisms of $P$ can be identified, via the section $s$, with the set of all smooth maps $\Sigma \rightarrow G$; the group structure is now simply pointwise multiplication. The subgroup $\mathcal{G}_{o}$ now consists of those $\phi \in \Sigma$ for which $\phi(o)=e$. The action of $\mathcal{G}$ on $\mathcal{A}$ is given by:

$$
\mathcal{A} \times \mathcal{G} \rightarrow \mathcal{A}:(A, \phi) \mapsto A \cdot \phi \stackrel{\text { def }}{=} \operatorname{Ad}\left(\phi^{-1}\right) A+\phi^{-1} d \phi .
$$

This action carries the sets $\mathcal{A}(\Theta)$ and $\mathcal{A}^{0}(\Theta)$ into themselves, and we have the moduli space

$$
\mathcal{M}(\Theta)=\mathcal{A}^{0}(\Theta) / \mathcal{G}
$$

6.2 The 2-forms $\Omega$ and $\Omega_{\Theta}$. We are interested in a certain 2-form $\Omega_{\Theta}$ on $\mathcal{A}(\Theta)$ which was introduced in [KS3]. The definition is:

$$
\Omega_{\Theta} \stackrel{\text { def }}{=} \Omega+\Omega_{\mathrm{ex}}+\Omega_{\nu}
$$

We shall explain now the definitions of the $2-$ forms $\Omega, \Omega_{\mathrm{ex}}$ and $\Omega_{\nu}$. The standard 2 -form $\Omega$ on $\mathcal{A}$ (as described in [AB]) is given by:

$$
\Omega(A, B) \stackrel{\text { def }}{=} \int_{\Sigma}\langle A \wedge B\rangle
$$

where $A$ and $B$ are $g$-valued 1-forms on $\Sigma$ and the product $\langle A \wedge B\rangle$ is the 2-form on $\Sigma$ given by $\langle A \wedge \bar{B}\rangle(X, Y)=\langle A(X), B(Y)\rangle_{g}-\langle A(Y), B(X)\rangle_{g}$.

Recall, from section 6.1, the loop $C$, part of which goes around $\partial \Sigma$. Let $A$ be a tangent vector to $\mathcal{A}$ (i.e. $A$ is a $\underline{g}$-valued 1 -form on $\Sigma$ ), and define

$$
\alpha:[0,1] \rightarrow \underline{g}: t \mapsto \alpha(t)=-\int_{0}^{t} \operatorname{Ad}\left(h_{s}^{-1}\right) A\left(C^{\prime}(s)\right) d s,
$$

where $s \mapsto h_{s}$ describes parallel transport along $C: h^{\prime}(s) h(s)^{-1}=-A\left(C^{\prime}(s)\right)$ with $h(0)=e$. It is known (and readily verifiable) that $\alpha(t)$ is the variation in $h_{t}$ corresponding to the variation $A$ in the connection $\omega$. Define $\beta:[0,1] \rightarrow G$ similarly with respect to a tangent vector $B$ to $\mathcal{A}$. The $2-$ form $\Omega_{\text {ex }}$ is defined by:

$$
\Omega_{\mathrm{ex}}(A, B) \stackrel{\text { def }}{=}-\frac{1}{2} \int_{0}^{1} \int_{0}^{1} \epsilon_{s t}\left\langle\alpha^{\prime}(s), \beta^{\prime}(t)\right\rangle d s d t,
$$

where

$$
\epsilon_{s t}= \begin{cases}1 & \text { if } s \leq t \\ -1 & \text { if } s>t\end{cases}
$$

Next, the 2-form $\Omega_{\nu}$ on $\mathcal{A}(\Theta)$ is defined by:

$$
\left.\Omega_{\nu}\right|_{\omega}(A, B)=-\frac{1}{2}\left\langle\alpha(1),(\operatorname{Ad}(c)-1)^{-1} \beta(1)\right\rangle+\frac{1}{2}\left\langle\beta(1),(\operatorname{Ad}(c)-1)^{-1} \alpha(1)\right\rangle,
$$

where $c=h(C ; \omega), C$ being the loop for $\partial \Sigma$ as described in Sect. 6.1, and $\alpha, \beta$ ar as in (6.2.3). The terms involving $(\operatorname{Ad}(c)-1)^{-1}$ are understood by setting $(\operatorname{Ad}(c)-1)^{-1}$ to be 0 on $\operatorname{ker}[\operatorname{Ad}(c)-1]=[(\operatorname{Ad}(c)-1)(g)]^{\perp}$. Thus all terms on the right of the definition (6.2.1) of $\Omega_{\Theta}$ have been specified.

6.3 The results of [KS3]. For ease of reference, we shall record here certain facts relating to $\mathcal{M}(\Theta)$ and $\Omega_{\Theta}$, including most of the results of [KS3]. 
(i) The 2-form $\Omega_{\Theta}$ is equivariant under the action of $\mathcal{G}$. Let $A, B \in T_{\omega} \mathcal{A}(\Theta)$ (an element of $T_{\omega} \mathcal{A}(\Theta)$ is, by definition, of the form $\partial \omega_{t} /\left.\partial t\right|_{t=0}$ where $t \mapsto \omega_{t} \in \mathcal{A}(\Theta)$ is such that $(t, p) \mapsto \omega_{t}(p)$ is smooth and $\left.\omega_{0}=\omega\right)$ and suppose that $A$ or $B$ is tangent to the $\mathcal{G}$-orbit through $\omega$, in the sense that it is equal to $\partial\left(\omega \cdot \phi_{t}\right) /\left.\partial t\right|_{t=0}$ for some path $t \mapsto \phi_{t} \in \mathcal{G}$ with $(t, p) \mapsto \phi_{t}(p)$ smooth and $\phi_{0}=i d$. Then $\Omega_{\Theta}(A, B)=0$ (Proposition 5.1 in [KS3].)

(ii) The holonomy representation

$$
\mathcal{H}: \omega \mapsto\left(h\left(A_{1} ; \omega\right), h\left(B_{1} ; \omega\right), \ldots, h\left(A_{\mathrm{g}} ; \omega\right), h\left(B_{\mathrm{g}} ; \omega\right), h(C ; \omega)\right) \in G^{2 \mathrm{~g}} \times \Theta,
$$

induces a bijection $\mathcal{A}(\Theta) / \mathcal{G}_{0} \rightarrow \Pi^{-1}(e)$, where $\mathcal{G}_{0}$ is the subgroup of $\mathcal{G}$ consisting of all $\phi$ with $\phi(o)=e$, and

$$
\Pi: G^{2 \mathrm{~g}} \times \Theta \rightarrow G:\left(a_{1}, b_{1}, \ldots, a_{\mathrm{g}}, b_{\mathrm{g}}, c\right) \mapsto c b_{\mathrm{g}}^{-1} a_{\mathrm{g}}^{-1} b_{\mathrm{g}} a_{\mathrm{g}} \cdots b_{1}^{-1} a_{1}^{-1} b_{1} a_{1}
$$

The group $G$ acts on the right of $\Pi^{-1}(e)$ by conjugation, and the holonomy mapping $\mathcal{H}$ is $\mathcal{G}-G$ equivariant with respect to the homomorphism $\mathcal{G} \rightarrow G: \phi \mapsto \phi(o)$, and induces a bijection of the full moduli spaces

$$
\mathcal{M}(\Theta) \rightarrow \Pi^{-1}(e) / G
$$

(iii) There is a dense open subset $\mathcal{D}$ of $G$ such that for every conjugacy class $\Theta$ passing through any point in $\mathcal{D}$, the set $\Pi^{-1}(e)$ is a smooth submanifold of $G^{2 \mathrm{~g}} \times \Theta$.

(iv) There is a 2-form $\bar{\Omega}_{o, \Theta}$ on $G^{2 \mathrm{~g}} \times \Theta$ whose restriction to $\Pi^{-1}(e)$ pulls back to $\Omega_{\Theta}$ by the holonomy map $\mathcal{H}$.

(v) If $\alpha \in \Pi^{-1}(e)$ and $A, B \in T_{\alpha} \Pi^{-1}(e)$ (this being taken, by definition, to be $\left.\operatorname{ker} d \Pi_{\alpha}\right)$ then $\bar{\Omega}_{o, \Theta}(A, B)$ is 0 if $A$ or $B$ is tangent to the $G$-orbit through $\alpha$; moreover, $\bar{\Omega}_{o, \Theta}$ is $G$-equivariant. In this sense $\bar{\Omega}_{o, \Theta}$ descends to a 2-form, also denoted $\bar{\Omega}_{\Theta}$, on $\Pi^{-1}(e) / G$. (Theorem 3.6 in [KS3])

(vi) The 2-form $\bar{\Omega}_{\Theta}$ is closed (i.e. $\bar{\Omega}_{o, \Theta}$ on $\Pi^{-1}(e)$ is closed); it is non-degenerate on the smooth part of $\Pi^{-1}(e) / G$ for all $\Theta$ passing through a dense open subset of $a$ certain neighborhood of the identity in $G$ (Theorem 4.1 in [KS3]). By the 'generic (or smooth) part of $\Pi^{-1}(e) / G$ ' we mean the subset of points corresponding to the points on $\Pi^{-1}(e)$ where the derivative $d \Pi$ is surjective and where the isotropy of the $G$-action is minimal within any component. (Sect. 7.4 below gives a more detailed explanation of 'generic part' of $\mathcal{M}(\theta)$ )

(vii) Formula specifying $\bar{\Omega}_{\Theta}$ : Let $\left(\alpha H^{(1)}, c C^{(1)}\right),\left(\alpha H^{(2)}, c C^{(2)}\right) \in T_{(\alpha, c)}\left(G^{2 g} \times \Theta\right)$; then

$$
\begin{aligned}
& \bar{\Omega}_{o, \Theta}\left(\left(\alpha H^{(1)}, c C^{(1)}\right),\left(\alpha H^{(1)}, c C^{(1)}\right)\right) \\
& =\frac{1}{2} \sum_{1 \leq i, k \leq 4 \mathrm{~g}} \epsilon_{i k}\left\langle\operatorname{Ad}\left(\alpha_{i-1} \cdots \alpha_{1}\right)^{-1} H_{i}^{(1)}, \operatorname{Ad}\left(\alpha_{k-1} \cdots \alpha_{1}\right)^{-1} H_{k}^{(1)}\right\rangle_{\underline{g}} \\
& -\frac{1}{2}\left\langle\left(\operatorname{Ad} c^{-1}-1\right)^{-1} C^{(1)},\left(\operatorname{Ad} c^{-1}-\operatorname{Ad} c\right)\left(\operatorname{Ad} c^{-1}-1\right)^{-1} C^{(2)}\right\rangle_{\underline{g}}
\end{aligned}
$$

where 


$$
\epsilon_{i k}=\left\{\begin{array}{ll}
1 & \text { if } i<k \\
0 & \text { if } i=k \\
-1 & \text { if } i>k
\end{array},\right.
$$

and we have used the convenient if unusual notation of setting

$$
\alpha=\left(\alpha_{1}, \alpha_{2}, \alpha_{5}, \alpha_{6}, \ldots, \alpha_{4 g-3}, \alpha_{4 g-2}\right)
$$

and

$$
\alpha_{j+2}=\alpha_{j}^{-1} \text { for all } j \in J \stackrel{\text { def }}{=}\{1,2,5,6, \ldots, 4 \mathrm{~g}-3,4 \mathrm{~g}-2\}
$$

and, analogously, $H^{(r)}=\left(H_{1}^{(r)}, H_{2}^{(r)}, H_{5}^{(r)}, H_{6}^{(r)}, \ldots, H_{4 \mathrm{~g}-3}^{(r)}, H_{4 \mathrm{~g}-2}^{(r)}\right)$, and

$$
H_{j+2}=-\operatorname{Ad}\left(\alpha_{j}\right) H_{j} \text { for all } j \in J \text {. }
$$

In (6.3.4), the terms (Ad $\left.c^{-1}-1\right)^{-1}$ are meaningful because $c C^{(1)}, c C^{(2)} \in T_{c} \Theta$ and, as is readily seen, $c^{-1} T_{c} \Theta=\left(\operatorname{Ad} c^{-1}-1\right)(\underline{g})$.

Note that (6.3.4) specifies the 2-form $\bar{\Omega}_{o, \Theta}$ on all of $G^{2 \mathrm{~g}} \times \Theta$; the 2 -form $\bar{\Omega}_{\Theta}$ is obtained from its restriction to $\Pi^{-1}(e)$.

6.4 The sense in which $\mathcal{H}$ induces $\bar{\Omega}_{\Theta}$ from $\Omega_{\Theta}$. We wish to make a technical remark here concerning Sect. 6.3(iv). A precise statement of 6.3(iv) is as follows. Let $(\alpha, c) \in$ $\Pi^{-1}(e) \subset G^{2 \mathrm{~g}} \times \Theta$, and consider vectors $v_{1}, v_{2} \in T_{(\alpha, c)}\left(G^{2 \mathrm{~g}} \times \Theta\right)$ which are tangent to smooth paths lying entirely on $\Pi^{-1}(e)$. It has been shown in Sects. 4.1-4.4 of [KS2] that:

(a) there are paths $[0,1] \rightarrow \mathcal{A}^{0}(\Theta): t \mapsto \omega_{t}^{i}$, with $i=1,2$, such that $(t, p) \mapsto \omega_{t}^{i}(p)$ is smooth and $\mathcal{H}\left(\omega_{t}^{i}\right)$ is initially tangent to $v_{i}$, for $i=1,2$.

(b) $\Omega_{\Theta}\left(V_{1}, V_{2}\right)=\bar{\Omega}_{o, \Theta}\left(v_{1}, v_{2}\right)$, where $V_{i}=\partial \omega_{i}^{t} /\left.\partial t\right|_{t=0}$, for $i=1,2$.

In view of Sect. 6.3(i), it is then reasonable to say that $\bar{\Omega}_{o, \Theta}$ gives the 2-form on $\Pi^{-1}(e) \simeq \mathcal{A}(\Theta) / \mathcal{G}_{o}$ (as in Sect. 6.3(ii)) induced by $\Omega_{\Theta}$, and hence that the 2 -form $\bar{\Omega}_{\Theta}$ for $\mathcal{M}(\Theta)$ is induced by $\Omega_{\Theta}$. However, to make this a strictly logical conclusion one should verify that if $A, B \in T_{\omega} \mathcal{A}^{0}(\Theta)$ are such that $\mathcal{H}^{\prime}(\omega) A$ or $\mathcal{H}^{\prime}(\omega) B$ (the derivatives being pointwise partial derivatives, for instance $\mathcal{H}^{\prime}(\omega) A=\partial \mathcal{H}\left(\omega_{t}\right) /\left.\partial t\right|_{t=0}$ if $t \mapsto \omega_{t} \in \mathcal{A}^{0}(\Theta)$ is such that $(t, p) \mapsto \omega_{t}(p)$ is smooth and $\left.\partial \omega_{t}(p) /\left.\partial t\right|_{t=0}=A\right)$ is 0 then $\Omega_{\Theta}(A, B)=0$. For the case of closed surfaces this has been proven in Theorem 5.9.1(i) of [Se6] by showing that $\mathcal{H}^{\prime}(\omega) A$ is 0 if and only if $A$ is tangent to the $\mathcal{G}_{o}$-orbit through $\omega$. We expect that this result (and, with minor modifications, its proof) holds for surfaces with boundary. A full treatment of this issue in the setting of compact surfaces with any number of boundary components is postponed to a future investigation. For the purposes of this paper it will suffice to take the relationship of $\Omega_{\Theta}$ and $\vec{\Omega}_{\Theta}$ as specified above by (a) and (b).

\section{Properties of $\bar{\Omega}_{\Theta}$, a Determinant Identity, and Non-Degeneracy for $\overline{\boldsymbol{\Omega}}_{\boldsymbol{\theta}}$}

The goal of this section is to prove that $\bar{\Omega}_{\Theta}$ is non-degenerate. For this we shall first prove a determinant identity ((7.8.1)) which will be useful again in Sects. 8 and 9. 
7.1 Notation. We record here some notation some of which has already been used in Sect. 6. We work with a conjugacy class $\Theta$ in $G$, and we use the map

$$
\Pi: G^{2 \mathrm{~g}} \times \Theta \rightarrow G:\left(a_{1}, b_{1}, \ldots, a_{\mathrm{g}}, b_{\mathrm{g}}, c\right) \mapsto c b_{\mathrm{g}}^{-1} a_{\mathrm{g}}^{-1} b_{\mathrm{g}} a_{\mathrm{g}} \cdots b_{1}^{-1} a_{1}^{-1} b_{1} a_{1} .
$$

The indexing set

$$
J \stackrel{\text { def }}{=}\{1,2,5,6, \ldots, 4 \mathrm{~g}-3,4 \mathrm{~g}-2\}
$$

is convenient to label a typical point in $G^{2 \mathrm{~g}} \times \Theta$ as

$$
\alpha=\left(\left\{\alpha_{j}\right\}_{j \in J}, c\right)
$$

Thus, comparing with the usual notation $\left(a_{1}, b_{1}, \ldots, a_{\mathrm{g}}, b_{\mathrm{g}}, c\right)$ we have set $\alpha_{1}=a_{1}$, $\alpha_{2}=b_{1}, \ldots, \alpha_{4 \mathrm{~g}-3}=a_{\mathrm{g}}, \alpha_{4 \mathrm{~g}-2}=b_{\mathrm{g}}$. As seen in the expression for $\bar{\Omega}_{o, \Theta}$ in (6.3.4), it is also convenient to introduce

$$
\alpha_{3}=\alpha_{1}^{-1}, \alpha_{4}=\alpha_{2}^{-1}, \ldots, \alpha_{4 g-1}=\alpha_{4 g-3}^{-1}, \alpha_{4 g}=\alpha_{4 g-2}^{-1}
$$

Correspondingly, a vector in $T_{(\alpha, c)}\left(G^{2 \mathrm{~g}} \times \Theta\right)$ has the form $\left(\left\{\alpha_{j} H_{j}\right\}_{j \in J}, c C\right)$, with $C \in c^{-1} T_{c} \Theta \subset \underline{g}$ (not to be confused with the loop $C$ itself), and we set

$$
H_{j+2}=-\left(\operatorname{Ad} \alpha_{j}\right) H_{j} \text { for } j \in J \text {. }
$$

It may be convenient to view the above notation as an expression of the imbedding $G^{2 \mathrm{~g}} \times \Theta \rightarrow G^{4 \mathrm{~g}} \times \Theta:\left(a_{1}, b_{1}, \ldots, a_{\mathrm{g}}, b_{\mathrm{g}}, c\right) \mapsto\left(a_{1}, b_{1}, a_{1}^{-1}, b_{1}^{-1}, \ldots, a_{\mathrm{g}}, b_{\mathrm{g}}, a_{\mathrm{g}}^{-1}, b_{\mathrm{g}}^{-1}, c\right)$.

The derivative of $\Pi$ at a point $(\alpha, c) \in G^{2 \mathrm{~g}} \times \Theta$ will be taken, by means of left translation, as a map $T_{(\alpha, c)}\left(G^{2 \mathrm{~g}} \times \Theta\right) \rightarrow \underline{g}$; it is given by

$$
d \Pi_{(\alpha, c)}(\alpha H, c C)=\sum_{i=1}^{4 \mathrm{~g}} f_{i-1}^{-1} H_{i}+f_{4 \mathrm{~g}}^{-1} C
$$

wherein

$$
f_{i}=\operatorname{Ad}\left(\alpha_{i} \cdots \alpha_{1}\right)
$$

Alternatively,

$$
d \Pi_{(\alpha, c)}(\alpha H, c C)=\sum_{j \in J}\left(f_{j-1}^{-1}-f_{j+2}^{-1}\right) H_{j}+f_{4 \mathrm{~g}}^{-1} C .
$$

Here we have used the fact that for any $j \in J$,

$$
\operatorname{Ad}\left(f_{j+1}^{-1}\right) H_{j+2}=-\operatorname{Ad}\left(f_{j+2}^{-1}\right) H_{j} .
$$

This form is useful for determining the adjoint $d \Pi_{(\alpha, c)}^{*}$, which, again by left translations, we will take as a map $\underline{g} \rightarrow\left(\underline{g}^{2 \mathrm{~g}}\right) \oplus\left(c^{-1} T_{c} \Theta\right)$; it is given by:

$$
d \Pi_{(\alpha, c)}^{*} X=\left(\left\{\left(f_{j-1}-f_{j+2}\right) X\right\}_{j \in J}, \operatorname{pr}_{2 \mathrm{~g}+1} f_{4 \mathrm{~g}} X\right)
$$

where $\mathrm{pr}_{2 \mathrm{~g}+1}$ is the orthogonal projection $\underline{g} \rightarrow c^{-1} T_{c} \Theta$. For the orbit map

$$
\gamma_{(\alpha, c)}: G \rightarrow G^{2 g} \times \Theta: x \mapsto \gamma_{(\alpha, c)}(x)=\left(\left\{x \alpha_{j} x^{-1}\right\}_{j \in J}, x c x^{-1}\right)
$$


the derivative is the map $\underline{g} \rightarrow \underline{g}^{2 \mathrm{~g}} \oplus\left(c^{-1} T_{c} \Theta\right)$ given by

$$
\gamma_{(\alpha, c)}^{\prime}(\alpha H, c C)=\left(\left\{\left(\operatorname{Ad} \alpha_{j}^{-1}-1\right) H_{j}\right\}_{j \in J},\left(\operatorname{Ad} c^{-1}-1\right) C\right) .
$$

Here $C$ is of course an element of $c^{-1} T_{c} \Theta \subset \underline{g}$, and is not the loop $C$ of Sect. 6.1.

7.2 Interpretation of maps of the form $(\operatorname{Ad} c-1)^{-1}$. We record some simple facts, conventions, and observations which will be useful later in computations. For $c \in G$, let

$$
Z_{c} \stackrel{\text { def }}{=}\{X \in \underline{g}:(\operatorname{Ad} c) X=X\} \text {. }
$$

Since

$$
(\operatorname{Ad} c-1)^{*}=\operatorname{Ad} c^{-1}-1
$$

it follows that

$$
\left(\operatorname{Ad} c^{ \pm 1}-1\right)(\underline{g})=Z_{c}^{\perp}=c^{-1} T_{c} \Theta
$$

and $\left(\operatorname{Ad} c^{ \pm 1}-1\right)$ map $Z_{c}^{\perp}$ into itself isomorphically; by $\left(\operatorname{Ad} c^{ \pm 1}-1\right)^{-1}$ we shall mean the inverse of this map. We have encountered and shall encounter again the composite $\left(\operatorname{Ad} c^{-1}-\operatorname{Ad} c\right)\left(\operatorname{Ad} c^{-1}-1\right)^{-1}$. Observe that

$$
\operatorname{Ad} c^{-1}-\operatorname{Ad} c=-(\operatorname{Ad} c-1)\left(1+\operatorname{Ad} c^{-1}\right)=(1+\operatorname{Ad} c)\left(\operatorname{Ad} c^{-1}-1\right) .
$$

Thus (Ad $\left.c^{-1}-\operatorname{Ad} c\right)$ maps $\underline{g}$ into $Z_{c}^{\perp}$, and so $\left(\operatorname{Ad} c^{-1}-\operatorname{Ad} c\right)\left(\operatorname{Ad} c^{-1}-1\right)^{-1}$ maps $Z_{c}^{\perp}$ into itself. Splitting any $\bar{X} \in \underline{g}$ into a component in $Z_{c}$ and one in $Z_{c}^{\perp}$ shows that

$$
\left(\operatorname{Ad} c^{-1}-\operatorname{Ad} c\right)\left(\operatorname{Ad} c^{-1}-1\right)^{-1}\left(\operatorname{Ad} c^{-1}-1\right) X=\left(\operatorname{Ad} c^{-1}-\operatorname{Ad} c\right) X .
$$

From (7.2.4) we have, for any $C^{(1)} \in Z_{c}^{\perp}$ and $X \in \underline{g}$,

$$
\begin{aligned}
& \left\langle\left(\operatorname{Ad} c^{-1}-1\right)^{-1} C^{(1)},\left(\operatorname{Ad} c^{-1}-\operatorname{Ad} c\right) X\right\rangle_{\underline{g}} \\
& =-\left\langle C^{(1)},\left(1+\operatorname{Ad} c^{-1}\right) X\right\rangle_{\underline{g}} \\
& =\left\langle C^{(1)},(\operatorname{Ad} c-1)^{-1}\left(\operatorname{Ad} c^{-1}-\operatorname{Ad} c\right) X\right\rangle,
\end{aligned}
$$

where in the second equality we used the hypothesis that $C^{(1)} \in Z_{c}^{\perp}$ and the fact (following from (7.2.4)) that

$$
(\operatorname{Ad} c-1)^{-1}\left(\operatorname{Ad} c^{-1}-\operatorname{Ad} c\right) X=-\left(1+\operatorname{Ad} c^{-1}\right) X+\text { an element of } Z_{c} .
$$

The following result (similar to a result discussed in [KS1] for closed surfaces) says that the map $\Pi$, introduced in (6.3.2), plays a role somewhat analogous to that of a moment map.

7.3 .

Lemma 7.1. Let $(\alpha, c) \in \Pi^{-1}(e) \subset G^{2 \mathrm{~g}} \times \Theta,\left(\alpha H^{(1)}, c C\right) \in T_{(\alpha, c)}\left(G^{2 \mathrm{~g}} \times \Theta\right)$, and $X \in \underline{g}$. Then

$$
\bar{\Omega}_{o, \Theta}\left(\left(\alpha H^{(1)}, c C^{(1)}\right), \gamma_{(\alpha, c)}^{\prime}(X)\right)=\left\langle d \Pi_{(\alpha, c)}\left(\alpha H^{(1)}, c C^{(1)}\right),\left(\frac{1+A d c}{2}\right) X\right\rangle_{g}
$$


Proof. Recall that $\alpha=\left\{\alpha_{j}\right\}_{j \in J}$, and $\alpha_{j+2}=\alpha_{j}^{-1}$, for every $j \in J$, and $f_{i} \stackrel{\text { def }}{=} \operatorname{Ad}\left(\alpha_{i} \cdots \alpha_{1}\right)$ for every $i \in\{1, \ldots, 4 \mathrm{~g}\}$. Recall also the expression (7.1.12) for $\gamma_{(\alpha, c)}^{\prime}$. Then the expression for $\bar{\Omega}_{o, \Theta}$ in (6.3.4) gives:

$$
\begin{aligned}
& \bar{\Omega}_{o, \Theta}\left(\left(\alpha H^{(1)}, c C^{(1)}\right), \gamma_{(\alpha, c)}^{\prime}(X)\right) \\
& \stackrel{(7.2 .5)}{=} \frac{1}{2} \sum_{i, k=1}^{4 \mathrm{~g}} \epsilon_{i k}\left\langle f_{i-1}^{-1} H_{i}^{(1)}, f_{k-1}^{-1}\left(\operatorname{Ad} \alpha_{k}^{-1}-1\right) X\right\rangle \\
& -\frac{1}{2}\left\langle\left(\operatorname{Ad} c^{-1}-1\right)^{-1} C^{(1)},\left(\operatorname{Ad} c^{-1}-\operatorname{Ad} c\right) X\right\rangle \\
& \stackrel{(7.2 .6)}{=} \frac{1}{2} \sum_{i=1}^{4 \mathrm{~g}}\left\langle f_{i-1}^{-1} H_{i}^{(1)},\left\{\left(1-f_{i-1}^{-1}\right)+\left(f_{4 \mathrm{~g}}^{-1}-f_{i}^{-1}\right)\right\} X\right\rangle \\
& +\frac{1}{2}\left\langle C^{(1)},\left(1+\operatorname{Ad} c^{-1}\right) X\right\rangle \\
& \stackrel{(7.1 .9)}{=} \frac{1}{2} \sum_{j \in J}\left\langle H_{j}^{(1)},\left[f_{j-1}\left(1+f_{4 \mathrm{~g}}^{-1}-f_{j-1}^{-1}-f_{j}^{-1}\right)\right.\right. \\
& \left.\left.-f_{j+2}\left(1+f_{4 \mathrm{~g}}^{-1}-f_{j+1}^{-1}-f_{j+2}^{-1}\right)\right] X\right\rangle \\
& +\frac{1}{2}\left\langle(\operatorname{Ad} c) C^{(1)},(1+\operatorname{Ad} c) X\right\rangle \\
& =\sum_{j \in J}\left\langle H_{j}^{(1)},\left(f_{j-1}-f_{j+2}\right)\left(\frac{1+f_{4 \mathrm{~g}}^{-1}}{2}\right) X\right\rangle+\left\langle(\operatorname{Ad} c) C^{(1)},\left(\frac{1+\operatorname{Ad} c}{2}\right) X\right\rangle \\
& \stackrel{(7.1 .8)}{=}\left\langle d \Pi_{(\alpha, c)}\left(\alpha H^{(1)}, c C^{(1)}\right),\left(\frac{1+\operatorname{Ad} c}{2}\right) X\right\rangle,
\end{aligned}
$$

where the last line is obtained from the hypothesis that $(\alpha, c) \in \Pi^{-1}(e)$ which implies that $(\operatorname{Ad} c) f_{4 \mathrm{~g}}=1$.

7.4 The generic stratum of $\mathcal{M}(\Theta)$. The moduli space $\mathcal{M}(\Theta)$ is, in general, not a manifold. For the purposes of this paper we shall define the tangent space $T_{(\alpha, c)} \Pi^{-1}(e)$ to be ker $d \Pi_{(\alpha, c)}$; this could contain vectors which are not tangent to any paths in $\Pi^{-1}(e)$. By a $k$-form on $\Pi^{-1}(e)$ we mean the restriction of a $k$-form on $G^{2 \mathrm{~g}} \times \Theta$ to (the tangent spaces of) $\Pi^{-1}(e)$. A $G$-equivariant $k$-form $\eta$ on $\Pi^{-1}(e)$ for which $\eta\left(v_{1}, \ldots, v_{k}\right)=0$ whenever any $v_{i}$ is in the image of the derivative of the orbit map of the conjugation action of $G$ will be taken to be a $k$-form $\bar{\eta}$ over $\mathcal{M}(\theta)$. Such a form $\bar{\eta}$ is closed if it arises from a form $\eta^{\prime}$ on $G^{2 g} \times \Theta$ for which the restriction of $d \eta^{\prime}$ to the tangent spaces of $\Pi^{-1}(e)$ is 0 .

A simple argument based on the expressions for $d \Pi_{(\alpha, c)}^{*}$ and $\gamma_{(\alpha, c)}^{\prime}$ given in (7.1.10) and (7.1.12) shows that if $(\alpha, c) \in \Pi^{-1}(e)$ then

$$
\operatorname{ker} d \Pi_{(\alpha, c)}^{*}=\operatorname{ker} \gamma_{(\alpha, c)}^{\prime} \text {. }
$$

It has been shown by means of Sard's theorem in Sect. 3.2 of [KS4] that there is a dense open subset of $G$ such that if $\theta$ includes a point in this set then $\Pi$ is a submersion at every point on $\Pi^{-1}(e)$ and thus $\Pi^{-1}(e)$ is a smooth submanifold of $G^{2 g} \times \Theta$. We shall focus on such $\theta$, to be called generic $\theta$. 
For generic $\Theta$, we see by (7.4.1) that the conjugation action of $G$ on $\Pi^{-1}(e)$ is locally free. Standard results of transformation group theory ([Bre]) imply that in each connected component of $\Pi^{-1}(e)$ there is a dense open subset such that the quotient of this under the action of $G$ is a smooth manifold and the quotient map is a submersion. It follows that there is a dense open subset of $\mathcal{M}(\Theta)$ which is a manifold, to be called the generic part (or stratum) of $\mathcal{M}(\Theta)$, of dimension $(2 g-2) \operatorname{dim} G+\operatorname{dim}(\Theta)$. The quotient map $\Pi^{-1}(e) \rightarrow \mathcal{M}(\Theta)$ over this generic part is a submersion.

It will be convenient to define the following 'tangent space':

$$
T_{p} \mathcal{M}(\Theta) \stackrel{\text { def }}{=}\left(d \Pi_{p}^{*}(\underline{g})+\gamma_{p}^{\prime}(\underline{g})\right)^{\perp} \subset T_{p} \Pi^{-1}(e) .
$$

The subspace in (7.4.2) is equivariant under the conjugation action of $G$.

If $p$ projects to a point $\bar{p}$ in the generic part of $\mathcal{M}(\Theta)$ then the derivative at $p$ of the quotient map sets up

$$
\text { a } G \text {-invariant isomorphism } \mathrm{q}_{*} \text { of } T_{p} \mathcal{M}(\Theta) \text { onto } T_{\bar{p}} \mathcal{M}(\Theta) \text {. }
$$

Smooth $G$-equivariant $k$-forms on $\Pi^{-1}(e)$ which vanish on the directions $\gamma_{p}^{\prime}(\underline{g})$ correspond one-to-one by $\mathrm{q}^{*}$ to smooth $k$-forms on the generic part of $\mathcal{M}(\Theta)$.

$$
\begin{array}{r}
\text { We equip } T_{\bar{p}} \mathcal{M}(\Theta) \text { with the inner-product which makes } \\
\text { the isomorphism } q_{*} \text { in (7.4.2) an isometry. }
\end{array}
$$

Some of the discussion and results below carry over to more general $\Theta$, and we expect that suitable sharper versions of the results below exist for all conjugacy classes $\theta$. Towards this, note that for general $\Theta$ we may define $\mathrm{q}^{*} \bar{\Omega}_{\Theta}$ at $p \in \Pi^{-1}(e)$ to mean the restriction of $\bar{\Omega}_{o, \Theta}$ to $T_{p} \mathcal{M}(\Theta)$, and define det $\bar{\Omega}_{o, \Theta}$ to be the determinant of the restriction of $\bar{\Omega}_{o, \Theta}$ on $T_{p} \mathcal{M}(\Theta)$ with respect to any orthonormal basis in $T_{p} \mathcal{M}(\Theta)$ (Sect. 7.6 contains more on such determinants).

The case where $\theta$ consists of one point (this being any point lying necessarily in the center of $G$ ) becomes essentially identical to the theory for closed surfaces, i.e. the theory covered by [KS1].

7.5 .

Lemma 7.2. Split $T_{(\alpha, c)}\left(G^{2 \mathrm{~g}} \times \Theta\right)$ as a direct sum of orthogonal pieces

$$
T_{(\alpha, c)}\left(G^{2 \mathrm{~g}} \times \Theta\right)=d \Pi_{(\alpha, c)}^{*} \underline{(g)} \oplus \gamma_{(\alpha, c)}^{\prime} \underline{(g)} \oplus T_{(\alpha, c)} \mathcal{M}(\Theta) .
$$

Then, with respect to this decomposition, $\bar{\Omega}_{o, \Theta}$ has the 'matrix form'

$$
\left[\begin{array}{ccc}
* & Q & * \\
-Q^{\mathrm{t}} & 0 & 0 \\
* & 0 & q^{*} \bar{\Omega}_{\Theta}
\end{array}\right],
$$

where $Q$ is the bilinear map $d \Pi_{(\alpha, c)}^{*}(\underline{g}) \times \gamma_{(\alpha, c)}^{\prime}(\underline{g}) \rightarrow \mathbf{R}$ given by

$$
Q\left(Y, \gamma_{(\alpha, c)}^{\prime} X\right)=\left\langle d \Pi_{(\alpha, c)} Y, \frac{1+A d c}{2} X\right\rangle
$$

and $Q^{\mathrm{t}}$ is the bilinear map $\gamma_{(\alpha, c)}^{\prime}(\underline{g}) \times d \Pi_{(\alpha, c)}^{*}(\underline{g}) \rightarrow \mathbf{R}$ given on $\left(\gamma_{(\alpha, c)}^{\prime} X, Y\right)$ by the right side of (7.5.3). 
Proof. First we observe that $(\alpha, c) \in \Pi^{-1}(e)$ implies that $\Pi \circ \gamma_{(\alpha, c)}(x)=e$ for every $x \in G$, and so $d \Pi_{(\alpha, c)}^{*} \underline{(g)}$ and $\left.\gamma_{(\alpha, c)}^{\prime} \underline{g}\right)$ are indeed orthogonal. From (7.3.1) it follows that $\bar{\Omega}_{o, \Theta}\left(Y, \gamma_{(\alpha, c)}^{\prime} X\right)$ is 0 when $Y \in \operatorname{ker} d \Pi_{(\alpha, c)}$; since $d \Pi_{(\alpha, c)}^{*}(g)^{\perp}=\operatorname{ker} d \Pi_{(\alpha, c)}$, this explains the two zeros in the second column. The top block $Q$ in the second column is, by definition of the "matrix form",

$$
Q(A, B) \stackrel{\text { def }}{=} \bar{\Omega}_{\Theta}(A, B)
$$

and so the expression (7.5.3) for $Q$ is simply a restatement of (7.3.1). The second block in the first column now follows by skew-symmetry of $\bar{\Omega}_{o, \Theta}$. The bottom corner block follows from the fact that $\bar{\Omega}_{\Theta}$ is, by definition, the image of $\bar{\Omega}_{o, \Theta}$ under the isomorphism $\mathrm{q}_{*}$ of (7.4.4).

7.6 Determinants. Let $A: V \rightarrow W$ be a linear map between finite-dimensional innerproduct spaces. If $\operatorname{ker} A \neq\{0\}$, or if $V=\{0\}$, then we $\operatorname{define} \operatorname{det}(A)=0$. If $A(\neq 0)$ is an isomorphism onto its image $A(V)$, then by $\operatorname{det} A$ we shall mean the determinant of a matrix of $A$ relative to orthonormal bases in $V$ and $A(V)$. Thus $\operatorname{det} A$ is determined up to sign, but is otherwise independent of the choice of bases. Consideration of matrices shows that $\operatorname{det}\left(A \mid(\operatorname{ker} A)^{\perp}\right)=\operatorname{det}\left(A^{*} \mid A(V)\right)$. If $A$ is an isomorphism onto $W$, and if $B: W \rightarrow Z$ is a linear map into a finite dimensional inner-product space $Z$, then $\operatorname{det}(B A)=\operatorname{det}(B) \operatorname{det}(A)$.

If $P: V \times W \rightarrow \mathbf{R}$ is a bilinear form, where $V$ and $W$ are finite-dimensional inner-product spaces of equal dimension, then by det $P$ (determined up to sign) we shall mean the determinant of the matrix $\left[P\left(v_{i}, w_{j}\right)\right]$, where $\left\{v_{i}\right\}$ and $\left\{w_{j}\right\}$ are orthonormal bases in $V$ and $W$, respectively; det $P$ is taken to be 0 if $V$ and $W$ are 0 -dimensional. The determinant det $\bar{\Omega}_{\Theta}$, which we shall use below, is to be understood in this sense.

7.7 .

Lemma 7.3. Let $(\alpha, c) \in \Pi^{-1}(e)$. Denote by $\langle d \Pi \otimes d \Pi\rangle$ the bilinear form on $T_{(\alpha, c)}\left(G^{2 \mathrm{~g}} \times \Theta\right)$ defined by

$$
\langle d \Pi \otimes d \Pi\rangle(X, Y)=\left\langle d \Pi_{(\alpha, c)} X, d \Pi_{(\alpha, c)} Y\right\rangle_{\underline{g}},
$$

and let $\left\langle d \Pi \otimes(A d c) \mathrm{pr}_{2 \mathrm{~g}+1}\right\rangle$ be the bilinear form on $T_{(\alpha, c)}\left(G^{2 \mathrm{~g}} \times \Theta\right)$ given by

$$
\left\langle d \Pi \otimes(A d c) \operatorname{pr}_{2 \mathrm{~g}+1}\right\rangle(X, Y)=\left\langle d \Pi_{(\alpha, c)} X,(\operatorname{Ad} c) \mathrm{pr}_{2 \mathrm{~g}+1} Y\right\rangle,
$$

where $\mathrm{pr}_{2 \mathrm{~g}+1}$ is the projection of $T_{(\alpha, c)}\left(G^{2 \mathrm{~g}} \times \Theta\right)$ on the last factor $c^{-1} T_{c} \Theta$. Then, assuming that $c$ is not in the center of $G$, ( i.e. $\Theta$ consists of more than one point)

$$
\begin{aligned}
& \operatorname{det}\left(\bar{\Omega}_{o, \Theta}-\frac{1}{2}\langle d \Pi \otimes d \Pi\rangle+\frac{1}{2}\left\langle d \Pi \otimes(A d c) \operatorname{pr}_{2 g+1}\right\rangle\right) \\
& =\operatorname{det}\left(-\frac{1}{2}(A d c-1)^{-1}\left(A d c^{-1}-A d c\right)\left(A d c^{-1}-1\right)^{-1}\right),
\end{aligned}
$$

where $\left(A d c^{-1}-1\right)^{-1}$ and $(A d c-1)^{-1}$ are taken as maps from $\left(A d c^{-1}-1\right)(\underline{g})$ into itself. 
Proof. Let $(\alpha, c) \in \Pi^{-1}(e)$, and consider vectors $\left(\alpha H^{(1)}, c C^{(1)}\right),\left(\alpha H^{(2)}, c C^{(2)}\right) \in$ $T_{(\alpha, c)}\left(G^{2 \mathrm{~g}} \times \Theta\right)$. Recall the expression for $\bar{\Omega}_{o, \Theta}$ in (6.3.4):

$$
\begin{aligned}
\bar{\Omega}_{o, \Theta}\left(\left(\alpha H^{(1)}, c C^{(1)}\right),\left(\alpha H^{(2)}, c C^{(2)}\right)\right) \\
=\frac{1}{2} \sum_{i, k=1}^{4 \mathrm{~g}} \epsilon_{i k}\left\langle f_{i-1}^{-1} H_{i}^{(1)}, f_{k-1}^{-1} H_{k}^{(2)}\right\rangle \\
-\frac{1}{2}\left\langle\left(\operatorname{Ad} c^{-1}-1\right)^{-1} C^{(1)},\right. \\
\left.\left(\operatorname{Ad} c^{-1}-\operatorname{Ad} c\right)\left(\operatorname{Ad} c^{-1}-1\right)^{-1} C^{(2)}\right\rangle,
\end{aligned}
$$

where

$$
\begin{gathered}
f_{i}=\operatorname{Ad}\left(\alpha_{i} \cdots \alpha_{1}\right), \\
\left(\alpha_{1}, \ldots, \alpha_{4 \mathrm{~g}}\right)=\left(a_{1}, b_{1}, a_{1}^{-1}, b_{1}^{-1}, \ldots, a_{\mathrm{g}}, b_{\mathrm{g}}, a_{\mathrm{g}}^{-1}, b_{\mathrm{g}}^{-1}\right), \\
\alpha=\left(a_{1}, b_{1}, \ldots, a_{\mathrm{g}}, b_{\mathrm{g}}\right)=\left\{\alpha_{j}\right\}_{j \in J}, \\
J=\{1,2,5,6, \ldots, 4 \mathrm{~g}-3,4 \mathrm{~g}-2\} \\
H_{j+2}^{(r)}=-\left(\operatorname{Ad} \alpha_{j}\right) H_{j}^{(r)} \text { for every } j \in J
\end{gathered}
$$

all as explained in Sect. 7.1. From these we have

$$
\bar{\Omega}_{o, \Theta}\left(\left(\alpha H^{(1)}, c C^{(1)}\right),\left(\alpha H^{(2)}, c C^{(2)}\right)\right)=\sum_{j, k \in J}\left\langle H_{j}^{(1)}, \Omega_{j k} H_{k}^{(2)}\right\rangle+\left\langle C^{(1)}, \Omega_{2 g+12 g+1} C^{(2)}\right\rangle,
$$

wherein (upon using the relationship (7.1.9) between $H_{j+2}$ and $H_{j}$ in the expression for $\bar{\Omega}_{o, \Theta}$ in $\left.(7.7 .4 a)\right)$

$$
\Omega_{j k}=\frac{1}{2}\left[f_{j-1}\left(\epsilon_{j k} f_{k-1}^{-1}-\epsilon_{j k+2} f_{k+2}^{-1}\right)-f_{j+2}\left(\epsilon_{j+2 k} f_{k-1}^{-1}-\epsilon_{j k} f_{k+2}^{-1}\right)\right]
$$

and, using the second equality in (7.2.6),

$$
\Omega_{2 \mathrm{~g}+12 \mathrm{~g}+1}=-\frac{1}{2}(\operatorname{Ad} c-1)^{-1}\left(\operatorname{Ad} c^{-1}-\operatorname{Ad} c\right)\left(\operatorname{Ad} c^{-1}-1\right)^{-1} .
$$

Recall that

$$
J \stackrel{\text { def }}{=}\{1,2,5,6, \ldots, 4 \mathrm{~g}-3,4 \mathrm{~g}-2\},
$$

so that $J \cup(J+2)=\{1,2, \ldots, 4 \mathrm{~g}\}$.

So, from (7.7.5a), for $j, k \in J$ with $j+2<k$,

$$
\Omega_{j k}=\frac{1}{2}\left(f_{j-1}-f_{j+2}\right)\left(f_{k-1}^{-1}-f_{k+2}^{-1}\right) .
$$

On the other hand, from the expression for $d \Pi_{(\alpha, c)}$ in (7.1.8) we have:

$$
\begin{aligned}
& \langle d \Pi \otimes d \Pi\rangle\left(\left(\alpha H^{(1)}, c C^{(1)}\right),\left(\alpha H^{(2)}, c C^{(2)}\right)\right) \\
& \stackrel{(7.1 .8)}{=} \sum_{j, k \in J}\left\langle\left(f_{j-1}^{-1}-f_{j+2}^{-1}\right) H_{j}^{(1)},\left(f_{k-1}^{-1}-f_{k+2}^{-1}\right) H_{k}^{(2)}\right\rangle \\
& +\sum_{j \in J}\left\langle\left(f_{j-1}^{-1}-f_{j+2}^{-1}\right) H_{j}^{(1)},(\operatorname{Ad} c) C^{(2)}\right\rangle
\end{aligned}
$$




$$
+\left\langle(\operatorname{Ad} c) C^{(1)},\left(f_{k-1}^{-1}-f_{k+2}^{-1}\right) H_{k}^{(2)}\right\rangle+\left\langle C^{(1)}, C^{(2)}\right\rangle
$$

and

$$
\begin{aligned}
& \left\langle d \Pi \otimes(\operatorname{Ad} c) \mathrm{pr}_{2 g+1}\right\rangle\left(\left(\alpha H^{(1)}, c C^{(1)}\right),\left(\alpha H^{(2)}, c C^{(2)}\right)\right. \\
& \stackrel{(7.1 .8)}{=}\left\langle\sum_{j \in J}\left(f_{j-1}^{-1}-f_{j+2}^{-1}\right) H_{j}^{(1)},(\operatorname{Ad} c) C^{(2)}\right\rangle+\left\langle C^{(1)}, C^{(2)}\right\rangle .
\end{aligned}
$$

Thus, setting

$$
\Omega^{\prime} \stackrel{\text { def }}{=} \bar{\Omega}_{o, \Theta}-\frac{1}{2}\langle d \Pi \otimes d \Pi\rangle+\frac{1}{2}\left\langle d \Pi \otimes(\operatorname{Ad} c) \operatorname{pr}_{2 \mathrm{~g}+1}\right\rangle,
$$

we can write

$$
\begin{aligned}
& \Omega^{\prime}\left(\left(\alpha H^{(1)}, c C^{(1)}\right),\left(\alpha H^{(2)}, c C^{(2)}\right)\right) \\
& =\sum_{j, k \in J}\left\langle H_{j}^{(1)}, \Omega_{j k}^{\prime} H_{k}^{(2)}\right\rangle+\sum_{j \in J}\left\langle H_{j}^{(1)}, \Omega_{j 2 \mathrm{~g}+1}^{\prime} C^{(2)}\right\rangle \\
& +\sum_{k \in J}\left\langle C^{(2)}, \Omega_{2 \mathrm{~g}+1}^{\prime} H_{k}^{(2)}\right\rangle+\left\langle C^{(1)}, \Omega_{2 \mathrm{~g}+12 \mathrm{~g}+1}^{\prime} C^{(2)}\right\rangle
\end{aligned}
$$

where

$$
\Omega_{2 \mathrm{~g}+12 \mathrm{~g}+1}^{\prime}=-\frac{1}{2}(\operatorname{Ad} c-1)^{-1}\left(\operatorname{Ad} c^{-1}-\operatorname{Ad} c\right)\left(\operatorname{Ad} c^{-1}-1\right)^{-1}
$$

and, from (7.7.6), (7.7.7) and (7.7.8),

$$
\begin{array}{r}
\Omega_{j k}^{\prime}=\frac{1}{2}\left(f_{j-1}-f_{j+2}\right)\left(f_{k-1}^{-1}-f_{k+2}^{-1}\right)-\frac{1}{2}\left(f_{j-1}-f_{j+2}\right)\left(f_{k-1}^{-1}-f_{k+2}^{-1}\right)=0 \\
\text { for } j, k \in J \text { with } j+2<k
\end{array}
$$

and from (7.7.4b), (7.7.7) and (7.7.8),

$$
\Omega_{j 2 \mathrm{~g}+1}^{\prime}=0-\frac{1}{2}\left(f_{j-1}-f_{j+2}\right) \operatorname{Ad} c+\frac{1}{2}\left(f_{j-1}-f_{j+2}\right) \operatorname{Ad} c=0 \text { for } j \in J
$$

and, similarly,

$$
\Omega_{2 \mathrm{~g}+1 k}^{\prime}=-\frac{1}{2} \mathrm{Ad} c^{-1}\left(f_{k-1}^{-1}-f_{k+2}^{-1}\right) \text { for } k \in J .
$$

Thus the 'matrix' $\left[\Omega_{j k}^{\prime}\right]_{j, k \in J}$ for $\Omega^{\prime}$ has an upper triangular form:

$$
\Omega^{\prime}=\left[\begin{array}{cccccc}
D_{1} & 0 & 0 & \cdots & \cdots & 0 \\
* & D_{5} & 0 & \cdots & \cdots & 0 \\
* & * & \cdots & \cdots & \cdots & 0 \\
\cdots & \cdots & \cdots & D_{j} & \cdots & 0 \\
\vdots & \vdots & \vdots & \vdots & \vdots & \vdots \\
* & * & * & * & D_{4 \mathrm{~g}-3} & 0 \\
* & * & * & * & * & \Omega_{2 \mathrm{~g}+12 \mathrm{~g}+1}^{\prime}
\end{array}\right]
$$

where the diagonal entry $D_{j}$, for $j, j+1 \in J$, is given from (7.7.5a), (7.7.7) and (7.7.9), by 


$$
\begin{aligned}
D_{j} & \stackrel{\text { def }}{=}\left[\begin{array}{cc}
\Omega_{j j}^{\prime} & \Omega_{j j+1}^{\prime} \\
\Omega_{j+1}^{\prime} & \Omega_{j+1}^{\prime}{ }_{j+1}^{\prime}
\end{array}\right] \\
& =\left[\begin{array}{cc}
f_{j+2} f_{j-1}^{-1}-1 & f_{j+2} f_{j}^{-1} \\
-f_{j} f_{j-1}^{-1}+f_{j+3} f_{j-1}^{-1}-f_{j+3} f_{j+2}^{-1} & f_{j+3} f_{j}^{-1}-1
\end{array}\right] \\
& =\left[\begin{array}{cc}
\operatorname{Ad}\left(\alpha_{j}^{-1} \alpha_{j+1} \alpha_{j}\right)-1 & \operatorname{Ad}\left(\alpha_{j}^{-1} \alpha_{j+1}\right) \\
-\operatorname{Ad} \alpha_{j}+\operatorname{Ad}\left(\alpha_{j+1}^{-1} \alpha_{j}^{-1} \alpha_{j+1} \alpha_{j}\right)-\operatorname{Ad} \alpha_{j+1}^{-1} & \operatorname{Ad}\left(\alpha_{j+1}^{-1} \alpha_{j}^{-1} \alpha_{j+1}\right)-1
\end{array}\right]
\end{aligned}
$$

This factorizes (as observed in [KS1]) as

$$
D_{j}=\left(\begin{array}{cc}
a^{-1} & 0 \\
0 & b^{-1}
\end{array}\right)\left(\begin{array}{cc}
0 & 1 \\
-1 & a^{-1}-1
\end{array}\right)\left(\begin{array}{cc}
1 & 0 \\
b-1 & 1
\end{array}\right)\left(\begin{array}{ll}
a & 0 \\
0 & b
\end{array}\right),
$$

where $a=\operatorname{Ad} \alpha_{j}$ and $b=\operatorname{Ad} \alpha_{j+1}$. This implies that $\operatorname{det}\left(D_{j}\right)=1$. Combining this observation with the form for $\Omega^{\prime}$ given in (7.7.15), we have:

$$
\operatorname{det} \Omega^{\prime}=\operatorname{det} \Omega_{2 \mathrm{~g}+12 \mathrm{~g}+1}^{\prime} \prod_{j \in J^{\prime}} \operatorname{det} D_{j}=\operatorname{det} \Omega_{2 \mathrm{~g}+12 \mathrm{~g}+1}^{\prime} \text {, }
$$

where $J^{\prime}=\{j \in J: j+1 \in J\}=\{1,5, \ldots, 4 \mathrm{~g}-3\}$. Recalling the expression for $\Omega_{2 g+12 g+1}^{\prime}$ given in (7.7.11), the proof is complete.

7.8 .

Lemma 7.4. Let $(\alpha, c) \in \Pi^{-1}(e) \subset G^{2 \mathrm{~g}} \times \Theta$, and suppose that $\Pi: G^{2 \mathrm{~g}} \times \Theta \rightarrow G$ is a submersion at $(\alpha, c)$. Then

$$
\operatorname{det} \bar{\Omega}_{\Theta}=\left(\frac{\operatorname{det} \gamma_{(\alpha, c)}^{\prime}}{\operatorname{det} d \Pi_{(\alpha, c)}^{*}}\right)^{2} \operatorname{det}\left[(1-A d c)^{-1}\right]
$$

where $\operatorname{det}\left[(1-A d c)^{-1}\right]$ is the determinant of the map

$$
(1-\operatorname{Adc})^{-1}: Z_{c}^{\perp} \rightarrow Z_{c}^{\perp}
$$

with $Z_{c}^{\perp}$ being the subspace $(\operatorname{ker}(\operatorname{Adc}-1))^{\perp}=(1-\operatorname{Adc})(\underline{g})$.

In particular, $\bar{\Omega}_{\Theta}$ is non-degenerate on the smooth part of $\Pi^{-1}(e) / G$.

Proof. Recall from Lemma 7.5 the splitting $T_{(\alpha, c)}\left(G^{2 g} \times \Theta\right)$ as a direct sum of orthogonal pieces

$$
T_{(\alpha, c)}\left(G^{2 \mathrm{~g}} \times \Theta\right)=d \Pi_{(\alpha, c)}^{*} \underline{(g)} \oplus \gamma_{(\alpha, c)}^{\prime} \underline{(g)} \oplus T_{(\alpha, c)} \mathcal{M}(\Theta)
$$

and the corresponding 'matrix form' of $\bar{\Omega}_{o, \Theta}$ given by

$$
\left[\begin{array}{ccc}
* & Q & * \\
-Q^{\mathrm{t}} & 0 & 0 \\
* & 0 & \mathrm{q}^{*} \bar{\Omega}_{\Theta}
\end{array}\right]
$$

where

$$
Q\left(Y, \gamma_{(\alpha, c)}^{\prime} X\right)=\left\langle d \Pi_{(\alpha, c)} Y, \frac{1+\operatorname{Ad} c}{2} X\right\rangle
$$


for every $Y \in d \Pi_{(\alpha, c)}^{*}(\underline{g})$, and $\mathrm{q}_{*}: T_{(\alpha, c)} \mathcal{M}(\Theta) \rightarrow T_{\overline{(\alpha, c)}} \mathcal{M}(\Theta)$ is as explained in Sect. 7.4.

Note that in the decomposition (7.8.2), the sum of the second and third summands is $\operatorname{ker} d \Pi_{(\alpha, c)}$. Using this we see that the 'matrix' for $\frac{1}{2}\langle d \Pi \otimes d \Pi\rangle$ at $(\alpha, c)$ has the form

$$
\left[\begin{array}{lll}
* & 0 & 0 \\
0 & 0 & 0 \\
0 & 0 & 0
\end{array}\right]
$$

and the matrix for $\frac{1}{2}\left\langle d \Pi \otimes(\operatorname{Ad} c) \operatorname{pr}_{2 g+1}\right\rangle$ at $(\alpha, c)$ has the form

$$
\left[\begin{array}{ccc}
* & Q_{1} & * \\
0 & 0 & 0 \\
0 & 0 & 0
\end{array}\right]
$$

where $Q_{1}$ is the bilinear map $d \Pi_{(\alpha, c)}^{*}(\underline{g}) \times \gamma_{(\alpha, c)}^{\prime} \underline{(g)} \rightarrow \mathbf{R}$ given by

$$
Q_{1}\left(Y, \gamma_{(\alpha, c)}^{\prime} X\right)=\frac{1}{2}\left\langle d \Pi_{(\alpha, c)} Y,(\operatorname{Ad} c)\left(\operatorname{Ad} c^{-1}-1\right) X\right\rangle .
$$

Recall the bilinear form $\Omega^{\prime}$ on $T_{(\alpha, c)}\left(G^{2 g} \times \Theta\right)$ given in (7.7.9):

$$
\Omega^{\prime} \stackrel{\text { def }}{=} \bar{\Omega}_{o, \Theta}-\frac{1}{2}\langle d \Pi \otimes d \Pi\rangle+\frac{1}{2}\left\langle d \Pi \otimes(\operatorname{Ad} c) \mathrm{pr}_{2 \mathrm{~g}+1}\right\rangle .
$$

From the preceding observations we see that, relative to the splitting of $T_{(\alpha, c)}\left(G^{2 \mathrm{~g}} \times \Theta\right)$ as in (7.8.1), $\Omega^{\prime}$ has the matrix form:

$$
\left[\begin{array}{ccc}
* & Q_{2} & * \\
-Q^{\mathfrak{t}} & 0 & 0 \\
0 & * & \mathrm{q}^{*} \bar{\Omega}_{\Theta}
\end{array}\right]
$$

where $Q_{2}=Q+Q_{1}$ is the bilinear map $\left.d \Pi_{(\alpha, c)}^{*}(\underline{g}) \times \gamma_{(\alpha, c)}^{\prime} \underline{g}\right) \rightarrow \mathbf{R}$ given by

$$
Q_{2}\left(Y, \gamma_{(\alpha, c)}^{\prime} X\right)=\left\langle d \Pi_{(\alpha, c)} Y, X\right\rangle=\left\langle Y, d \Pi_{(\alpha, c)}^{*} X\right\rangle
$$

and $-Q^{\mathrm{t}}$ is the bilinear map $\gamma_{(\alpha, c)}^{\prime}(\underline{g}) \times d \Pi_{(\alpha, c)}^{*}(\underline{g}) \rightarrow \mathbf{R}$ given by

$$
-Q^{\mathrm{t}}\left(\gamma_{(\alpha, c)}^{\prime} X, Y\right)=-\left\langle d \Pi_{(\alpha, c)} Y, \frac{1+\operatorname{Ad} c}{2} X\right\rangle .
$$

From (7.8.8) we have, as usual not worrying about signs,

$$
\operatorname{det} \Omega^{\prime}=\operatorname{det} Q_{2} \operatorname{det}\left(-Q^{t}\right) \operatorname{det} \bar{\Omega}_{\Theta},
$$

and the expressions for $Q_{2}$ and $-Q^{t}$ then imply that

$$
\operatorname{det} \Omega^{\prime}=\left\{\frac{\operatorname{det} d \Pi_{(\alpha, c)}^{*}}{\operatorname{det} \gamma_{(\alpha, c)}^{\prime}} \cdot \frac{\operatorname{det} d \Pi_{(\alpha, c)}^{*}}{\operatorname{det} \gamma_{(\alpha, c)}^{\prime}} \operatorname{det}\left(-\frac{1+\operatorname{Ad} c}{2}\right)\right\} \operatorname{det} \bar{\Omega}_{\Theta} .
$$

Now decomposing $\underline{g}$ as the orthogonal sum of $Z_{c}=\operatorname{ker}(\operatorname{Ad} c-1)$ and $Z_{c}^{\perp}$, we have

$$
\operatorname{det}\left(\frac{1+\operatorname{Ad} c}{2}\right)=\operatorname{det}\left(\frac{1+\operatorname{Ad} c}{2} \mid Z_{c}^{\perp} \rightarrow Z_{c}^{\perp}\right) .
$$


Combining the expression for det $\Omega^{\prime}$ given in (7.8.10) with that obtained earlier in (7.7.3), and using (7.8.11), we obtain

$$
\begin{aligned}
& \operatorname{det} \bar{\Omega}_{\Theta}=\left(\frac{\operatorname{det} \gamma_{(\alpha, c)}^{\prime}}{\operatorname{det} d \Pi_{(\alpha, c)}^{*}}\right)^{2} \frac{\operatorname{det}\left[(\operatorname{Ad} c-1)^{-1}\left(\operatorname{Ad} c-\operatorname{Ad} c^{-1}\right)\left(\operatorname{Ad} c^{-1}-1\right)^{-1}\right]}{\operatorname{det}\left((1+\operatorname{Ad} c) \mid Z_{c}^{\perp}\right)} \\
& \stackrel{(7.2 .4)}{=}\left(\frac{\operatorname{det} \gamma_{(\alpha, c)}^{\prime}}{\operatorname{det} d \Pi_{(\alpha, c)}^{*}}\right)^{2} \frac{\operatorname{det}(1-\operatorname{Ad} c)^{-1} \operatorname{det}\left((1+\operatorname{Ad} c) \mid Z_{c}^{\perp}\right)}{\operatorname{det}\left((1+\operatorname{Ad} c) \mid Z_{c}^{\perp}\right)}
\end{aligned}
$$

thus proving the determinant identity (7.8.1).

Recall from Sect. 6.3(iii) that there is a dense open subset $\mathcal{D} \subset G$ such that for every conjugacy class $\theta$ passing through any point of $\mathcal{D}, \Pi$ is a submersion at every point of the level set $\Pi^{-1}(e)$ and thus $\Pi^{-1}(e)$ is a smooth submanifold of $G^{2 \mathrm{~g}} \times \theta$. In Sect. 7.4 we saw that $\bar{\Omega}_{\Theta}$ is a smooth 2-form on a subset (which we call the generic part) of $\mathcal{M}(\Theta)$ which is a smooth manifold; as noted in Sect. 6.3(vi), it has been proven in [KS2] that $\bar{\Omega}_{\Theta}$ is a closed 2-form on this generic part of $\mathcal{M}(\Theta)$. Combining this with Lemma 7.8 we obtain the following result.

\section{9 .}

Theorem 5. The 2-form $\bar{\Omega}_{\Theta}$ is a symplectic structure on the generic part of $\mathcal{M}(\Theta)$ for every generic conjugacy class $\Theta$ in $G$.

\section{Limiting Quantum Yang-Mills and Symplectic Volume}

In this section $\Sigma$ is equipped with a Riemannian metric; i.e. $\Sigma$ is a compact connected smooth Riemannian manifold with one connected boundary component $\partial \Sigma$. We will prove that as $T \downarrow 0$ the measures $\mu_{T}^{\Theta}$ (given heuristically by (2.7.5) and rigorously in Sect. 5.3) converge, in a sense specified below, to a volume measure on $\mathcal{M}(\theta)=$ $\mathcal{A}^{0}(\Theta) / \mathcal{G}$ which corresponds locally to the symplectic structure $\bar{\Omega}_{\Theta}$ on $\mathcal{M}(\Theta)$ (if $\lambda$ is a symplectic 2 -form on a $2 \mathrm{~d}$-dimensional space, then the corresponding volume form $\operatorname{vol}_{\lambda}$ is the exterior power $\wedge^{d} \lambda / d$ !).

Recall that the measure $\mu_{T}^{\Theta}$, as constructed in Sect. 5.3, is a probability measure on a space $\overline{\mathcal{A}(\Theta) / \mathcal{G}_{o}}$. For each well-behaved loop $\kappa$ on $\Sigma$ based at $o$ there is a random variable $\omega \mapsto h(\kappa ; \omega) \in G$ which corresponds to the the holonomy of $\omega$ around $\kappa$. For our present purposes we shall not need any details of the definition of $\overline{\mathcal{A}(\theta) / \mathcal{G}_{o}}$, nor of the stochastic random holonomies, nor of what 'well-behaved' loops mean. What we shall need is summarized in the following special case of Theorem 5.4, which may be taken as a specification of $\mu_{T}^{\Theta}$, thus providing a choice of a starting point for our present discussions.

The triangulations of $\Sigma$ that we use are assumed to be 'admissible' in the sense of Sect. 4.1. Alternatively, for the purposes of the present section, we may work with any arbitrary triangulation of $\Sigma$ and consider $\mu_{T}^{\Theta}$ as being a measure defined by means of (8.1.1) below. 


\subsection{Loop expectation values for $\mu_{T}^{\ominus}$.}

Let $\mathcal{S}$ be a (well-behaved) triangulation of $\Sigma$, with the basepoint o as a 0 -simplex. Then for any loops $\kappa_{1}, \ldots, \kappa_{n}$ based at $o$ and consisting of oriented 1-simplices of $\mathcal{S}$, and any bounded measurable function $f$ on $G^{n}$,

$$
\begin{aligned}
& \int f\left(h\left(\kappa_{1} ; \omega\right), \ldots, h\left(\kappa_{n} ; \omega\right)\right) d \mu_{T}^{\Theta}(\omega) \\
& =N_{T}(\Theta)^{-1} \int f\left(x\left(\kappa_{1}\right), \ldots, x\left(\kappa_{n}\right)\right) \delta\left(x(C) c^{-1}\right) \\
& \prod_{j=1}^{m} Q_{T\left|\Delta_{j}\right|}\left(x\left(\partial \Delta_{j}\right)\right) d x_{e_{1}} \cdots d x_{e_{M}} d_{\Theta} c
\end{aligned}
$$

where the notation is as explained in Theorem 5.4. The normalizer $N_{T}(\Theta)$ is given by:

$$
N_{T}(\Theta)=\int_{G^{2 \mathrm{~g}}} Q_{T|\Sigma|}\left(c b_{\mathrm{g}}^{-1} a_{\mathrm{g}}^{-1} b_{\mathrm{g}} a_{\mathrm{g}} \cdots b_{1}^{-1} a_{1}^{-1} b_{1} a_{1}\right) d a d b d_{\Theta} c
$$

By appropriate subdivision of $\mathcal{S}$ and by joining $o$ to a vertex of each $\Delta_{j}$ by an appropriate curve $l_{j}$, each loop $\kappa$ in $\mathcal{S}$ based at $o$ can be expressed as a 'composite' of a sequence of the loops of the form $\bar{l}_{j} \cdot \Delta_{j} \cdot l_{j}$ and their reverses, and the loops $A_{i}, B_{i}, C$ and their reverses (by 'composite' here we include the operation of successively dropping edges which are traversed in opposite directions consecutively in the usual composite of curves). This has been described in more detail in Sect. 4.2. Each $x\left(\kappa_{i}\right)$ in (8.1.1) is then a product of the $x\left(l_{j}\right)^{-1} x\left(\partial \Delta_{j}\right)^{ \pm 1} x\left(l_{j}\right)$ and $x\left(A_{i}\right)^{ \pm 1}, x\left(B_{i}\right)^{ \pm 1}$, and $x(C)^{ \pm 1}$. Thus

$$
\text { we can express } f\left(x\left(\kappa_{1}\right), \ldots, x\left(\kappa_{n}\right)\right) \text { as } F\left(y_{\Delta_{1}}, \ldots, y_{\Delta_{m}},\left\{a_{i}, b_{i}\right\}, c\right)
$$

for some function $F$, where

$$
\begin{gathered}
y_{\Delta_{j}}=x\left(l_{j_{i}}\right)^{-1} x\left(\partial \Delta_{j}\right) x\left(l_{j}\right), \\
a_{i}=x\left(A_{i}\right), b_{i}=x\left(B_{i}\right), c=x(C) .
\end{gathered}
$$

Conversely, given $y_{\Delta_{1}}, \ldots, y_{\Delta_{m}},\left\{a_{i}, b_{i}\right\}, c$ in $G$, satisfying

$$
y_{\Delta_{m}} \cdots y_{\Delta_{1}}=c b_{\mathrm{g}}^{-1} a_{\mathrm{g}}^{-1} b_{\mathrm{g}} a_{\mathrm{g}} \cdots b_{1}^{-1} a_{1}^{-1} b_{1} a_{1} \text {. }
$$

there is an assignment $e \mapsto x_{e}$, with $x_{\bar{e}}=x_{e}^{-1}$ for every oriented 1-simplex $e$ of $\mathcal{S}$, such that (8.1.4) and (8.1.5) hold.

With this change of variables it follows, as for (4.4.5),

$$
\begin{aligned}
& \int f\left(h\left(\kappa_{1} ; \omega\right), \ldots, h\left(\kappa_{n} ; \omega\right)\right) d \mu_{T}^{\Theta}(\omega) \\
& =\frac{1}{N_{T}(\Theta)} \int_{G^{m} \times G^{28} \times \Theta} F\left(y_{\Delta_{1}}, \ldots, y_{\Delta_{m}},\left\{a_{i}, b_{i}\right\}, c\right) \\
& \cdot \delta\left(y_{\Delta_{m}} \cdots y_{\Delta_{1}}\left(c b_{\mathrm{g}}^{-1} a_{\mathrm{g}}^{-1} b_{\mathrm{g}} a_{\mathrm{g}} b_{1}^{-1} a_{1}^{-1} b_{1} a_{1}\right)^{-1}\right) d_{\Theta} c \\
& \prod_{i=1}^{\mathrm{g}} d a_{i} d b_{i} \prod_{j=1}^{m} Q_{T\left|\Delta_{j}\right|}\left(y_{\Delta_{i}}\right) d y_{\Delta_{j}},
\end{aligned}
$$


where the $\delta(\cdot)$ term means that any one of the $y_{\Delta_{j}}$ may be replaced in the integrand by the value which makes the argument in $\delta(\cdot)$ equal to $e$, and the corresponding integration $d y_{\Delta_{j}}$, along with the $\delta(\cdot)$-term, dropped from the integration.

Taking $T \downarrow 0$ we obtain the following lemma which is very close to Lemma 2.14 in [Se4]; since the sketch proof presented in [Se4] is sloppy enough to be viewed as incorrect we present a detailed argument here.

8.2 .

Lemma 8.5. Let $\kappa_{1}, \ldots, \kappa_{n}$ be loops based at o and composed of oriented 1-simplices of the triangulation $\mathcal{S}$, let $f$ be a continuous function on $G^{n}$, and let $F$ be associated to $f$ as described above in (8.1.3). Then

$$
\begin{aligned}
& \lim _{T \downarrow 0} \int f\left(h\left(\kappa_{1} ; \omega\right), \ldots, h\left(\kappa_{n} ; \omega\right)\right) d \mu_{T}^{\Theta}(\omega) \\
& =\lim _{T \rightarrow 0} N_{T}(\Theta)^{-1} \int F\left(e, \ldots, e,\left\{a_{i}, b_{i}\right\}, c\right) \\
& \cdot Q_{T|\Sigma|}\left(c b_{\mathrm{g}}^{-1} a_{\mathrm{g}}^{-1} b_{\mathrm{g}} a_{\mathrm{g}} \cdots b_{1}^{-1} a_{1}^{-1} b_{1} a_{1}\right) d a_{1} d b_{1} \cdots d a_{\mathrm{g}} d b_{\mathrm{g}} d_{\Theta} c
\end{aligned}
$$

provided that the limit on the right side exists and provided that the limit

$$
N_{0}(\Theta) \stackrel{\text { def }}{=} \lim _{T \rightarrow 0} \int_{G^{2 g}} Q_{T|\Sigma|}\left(c b_{\mathrm{g}}^{-1} a_{\mathrm{g}}^{-1} b_{\mathrm{g}} a_{\mathrm{g}} \cdots b_{1}^{-1} a_{1}^{-1} b_{1} a_{1}\right) d a_{1} d b_{1} \cdots d a_{\mathrm{g}} d b_{\mathrm{g}} d_{\Theta} c
$$

exists and is positive.

Proof. Let $\lambda_{T}$ be the Borel measure on $G^{m} \times G^{2 \mathrm{~g}} \times \Theta$ specified by requiring that for every continuous function $h$ on $G^{m} \times G^{2 \mathrm{~g}} \times \Theta$,

$$
\begin{array}{r}
\int h d \lambda_{T}=\int_{G^{m-1} \times G^{28 \times \Theta}} h\left(y_{1}, \ldots y_{m-1}, y_{m}^{\prime}, a_{1}, \ldots, b_{\mathrm{g}}, c\right) . \\
Q_{T\left|\Delta_{m}\right|}\left(y_{m}^{\prime}\right)\left(\prod_{j=1}^{m-1} Q_{T\left|\Delta_{j}\right|}\left(y_{j}\right) d y_{j}\right)\left(\prod_{i=1}^{\mathrm{g}} d a_{i} d b_{i}\right) d_{\Theta} c
\end{array}
$$

wherein

$$
y_{m}^{\prime}=\left\{\Pi\left(a_{1}, \ldots, b_{\mathrm{g}}, c\right)\right\}\left(y_{m-1} \cdots y_{1}\right)^{-1}
$$

and, as usual,

$$
\Pi\left(a_{1}, \ldots, b_{\mathrm{g}}, c\right)=c b_{\mathrm{g}}^{-1} a_{\mathrm{g}}^{-1} b_{\mathrm{g}} a_{\mathrm{g}} \cdots b_{1}^{-1} a_{1}^{-1} b_{1} a_{1} .
$$

By a change-of-variables argument, the role of $y_{m}^{\prime}$ (and of $\Delta_{m}$ ) can be replaced by that of $y_{j}^{\prime}$ (and of $\Delta_{j}$ ) in the integration (8.2.3).

The loop expectation value formula (8.1.6) says that

$$
\begin{aligned}
& \int f\left(h\left(\kappa_{1} ; \omega\right), \ldots, h\left(\kappa_{n} ; \omega\right)\right) d \mu_{T}^{\Theta}(\omega) \\
& =\frac{1}{N_{T}(\Theta)} \int F\left(y_{1}, \ldots, y_{m},\left\{a_{i}, b_{i}\right\}, c\right) d \lambda_{T}\left(\left\{y_{j}\right\},\left\{a_{i}, b_{i}\right\}, c\right) .
\end{aligned}
$$

So we estimate

$$
\mid \int F\left(y_{1}, \ldots y_{m},\left\{a_{i}, b_{i}\right\}, c\right) d \lambda_{T}\left(\left\{y_{j}\right\},\left\{a_{i}, b_{i}\right\}, c\right)
$$




$$
\begin{aligned}
& -\int_{m} F\left(e, \ldots, e,\left\{a_{i}, b_{i}\right\}, c\right) d \lambda_{T}\left(\left\{y_{j}\right\},\left\{a_{i}, b_{i}\right\}, c\right) \mid \\
& \leq \sum_{j=1}^{m} \int \mid F\left(e, \ldots, e, y_{j}, \ldots, y_{m},\left\{a_{i}, b_{i}\right\}, c\right)- \\
& F\left(e, \ldots, e, y_{j+1}, \ldots, y_{m},\left\{a_{i}, b_{i}\right\}, c\right) \mid d \lambda_{T}\left(\left\{y_{j}\right\},\left\{a_{i}, b_{i}\right\}, c\right)
\end{aligned}
$$

wherein the $j=m$ term is interpreted using $F\left(e, \ldots, e,\left\{a_{i}, b_{i}\right\}, c\right)$ as the second term in the integrand.

Let $\epsilon>0$. By (uniform) continuity of $F$, there is a neighborhood $U_{\epsilon}$ of $e$ such that, for any $j \in\{1, \ldots, m\}$, if $y_{j} \in U_{\epsilon}$ then the integrand on the right side in (8.2.7) is less than $\epsilon$. Thus, since the total mass of $\lambda_{T}$ is $N_{T}(\Theta)$,

$$
\text { left side of }(8.2 .7) \leq m \epsilon \cdot N_{T}(\Theta)+2\|F\|_{\text {sup }} \sum_{j=1}^{m} \lambda_{T}\left(S_{j, \epsilon}\right),
$$

where

$$
S_{j, \epsilon} \stackrel{\text { def }}{=}\left\{\left(y_{1}, \ldots, y_{m},\left\{a_{i}, b_{i}\right\}, c\right) \in G^{m+2 g} \times \Theta: y_{j} \notin U_{\epsilon}\right\} .
$$

Using the convolution property $\int Q_{t}(a b) Q_{s}\left(b^{-1} c\right) d a d b=Q_{t+s}(a c)$, the conjugation invariance of $Q_{t}$, and $\int_{G} Q_{t}(x) d x=1$, we have

$$
\begin{aligned}
\lambda_{T}\left(S_{j, \epsilon}\right) & =\int_{y \notin U_{\epsilon}} Q_{T\left|\Delta_{j}\right|}(y) Q_{T|\Sigma|-T\left|\Delta_{j}\right|}\left(I\left(a_{1}, \ldots, b_{\mathrm{g}}, c\right) y^{-1}\right) d y d a_{1} \ldots d b_{\mathrm{g}} d_{\Theta} c \\
& \leq \sup _{y \notin U_{\epsilon}} Q_{T\left|\Delta_{j}\right|}(y) .
\end{aligned}
$$

Since $\lim _{t \downarrow 0} \sup _{y \notin U} Q_{t}(y)=0$ for any neighborhood $U$ of $e$, we can divide (8.2.8) by $N_{T}(\Theta)$, let $T \downarrow \delta$, and use the hypothesis that $N_{0}(\Theta)$ exists and is positive to conclude that (8.2.1) holds.

For the following, recall from section 8.3 that for any $\Theta$ which passes through a certain dense open subset of $G$, the map $\Pi: G^{2 \mathrm{~g}} \times \Theta \rightarrow G$ is a submersion at every point of $\Pi^{-1}(e)$.

8.3 .

Theorem 6. Assume that

(i) $\Theta$ is such that $\Pi$ is a submersion at every point of $\Pi^{-1}(e)$

(ii) $\Pi^{-1}(e)$ has a dense open subset $\Pi^{-1}(e)^{0}$ on which the isotropy of the conjugation action of $G$ is $Z(G)$

(iii) $\operatorname{vol}_{\bar{\Omega}_{\theta}}\left(\mathcal{M}(\Theta)^{0}\right)<\infty$, where $\mathcal{M}(\Theta)^{0}$ is the projection of $\Pi^{-1}(e)^{0}$ onto $\mathcal{M}(\Theta)$ by the projection map $\Pi^{-1}(e) \rightarrow \Pi^{-1}(e) / G \simeq \mathcal{M}(\Theta)$ ( as in (6.3.3)), and vol $\bar{\Omega}_{\Theta}$ is the volume with respect to the symplectic structure $\bar{\Omega}_{\Theta}$ on $\mathcal{M}(\Theta)^{0}$.

(iv) $\kappa_{1}, \ldots, \kappa_{n}$ are loops on $\Sigma$ based at $o$, as in section 8.1.

Then for any continuous function $f$ on $G^{n}$ which is invariant under the conjugation action of $G$, 


$$
\begin{aligned}
& \lim _{T \downarrow 0} \int f\left(h\left(\kappa_{1} ; \omega\right), \ldots, h\left(\kappa_{n} ; \omega\right)\right) d \mu_{T}^{\Theta}(\omega) \\
& =\frac{1}{\operatorname{vol}_{\bar{\Omega}_{\Theta}}\left(\mathcal{M}(\Theta)^{0}\right)} \int_{\mathcal{M}(\Theta)^{0}} f\left(h\left(\kappa_{1} ; \omega\right), \ldots, h\left(\kappa_{n} ; \omega\right)\right) d v o \bar{\Omega}_{\Theta}([\omega])
\end{aligned}
$$

Proof. In view of the limiting formulas given in Lemma 8.2, we shall focus on computing $\lim _{t \rightarrow 0} \int_{G^{28} \times \Theta} H\left(a_{1}, \ldots, b_{\mathrm{g}}, c\right) Q_{t}\left(\Pi\left(a_{1}, \ldots, b_{\mathrm{g}}, c\right)\right) d a_{1} \ldots d b_{\mathrm{g}} d_{\Theta} c$, for continuous functions $H$.

By the submersivity hypothesis, $\Pi^{-1}(e)$ is a submanifold of $G^{2 g} \times \theta$. Picking any point $x_{*} \in \Pi^{-1}(e)$ we have, again by submersivity of $\Pi$ at $x_{*}$, a coordinate system $\chi$ on a neighborhood $W$ of $x_{*}$ in $G^{2 \mathrm{~g}} \times \Theta$ and a coordinate system on a neighborhood $U$ of $\Pi\left(x_{*}\right)$ in $G$ such that $\Pi(W)=U$ and $\Pi \mid W$ corresponds, in the coordinates, to projection on the first $\operatorname{dim}(G)$ coordinates.

Let

$$
V=(\Pi \mid W)^{-1}(e)=\Pi^{-1}(e) \cap W
$$

Thus, taking the coordinate system $\chi$ such that $\chi(W)$ is a cube, there is a diffeomorphism

$$
\Phi: U \times V \rightarrow W
$$

such that $\Pi \circ \Phi: U \times V \rightarrow U$ is the projection on the first factor. Therefore, writing

$$
w=\Phi(u, v),
$$

the derivative $d \Phi_{(u, v)}: g \oplus T_{v} \Pi^{-1}(e) \rightarrow T_{\Phi(u, v)} W=d \Pi_{w}^{*}(\underline{g})+\operatorname{ker} d \Pi_{w}$ can be expressed as a matrix of the form

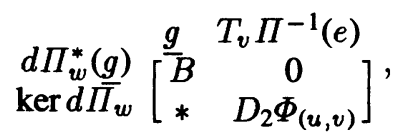

where $D_{2} \Phi_{(u, v)}: T_{v} \Pi^{-1}(e) \rightarrow \operatorname{ker} d \Pi_{\Phi(u, v)}$ is the partial deriative of $\Phi$ in the second variable, and $B^{-1}$ is the restriction of $d \Pi_{w}$ to $d \Pi_{w}^{*}(g)=\left(\operatorname{ker} d \Pi_{w}\right)^{\perp}$.

It should be noted that the diagonal blocks listed in (8.3.2) are indeed 'square blocks'. Moreover, $\operatorname{det} B^{-1}=\operatorname{det} d \Pi_{w}^{*}$. Consequently,

$$
\left|\operatorname{det} d \Phi_{(u, v)}\right|=\frac{\left|\operatorname{det} D_{2} \Phi_{(u, v)}\right|}{\left|\operatorname{det} d \Pi_{\Phi(u, v)}^{*}\right|} .
$$

Let $H$ be a continuous function on $G^{2 \mathrm{~g}} \times \Theta$ with compact support contained in $W$. For the following computations it is necessary to bear in mind that we use the unitmass invariant measures on $G$ and $\Theta$, and these differ by constant volume factors from the respective Riemannian volume measures. Then, upon using (8.3.3), we have the following change-of-variables formula

$$
\begin{aligned}
\operatorname{vol}(G)^{2 \mathrm{~g}} \operatorname{vol}(\Theta) & \int_{G^{28} \times \Theta} H\left(a_{1}, \ldots, b_{\mathrm{g}}, c\right) Q_{t}\left(\Pi\left(a_{1}, \ldots, b_{\mathrm{g}}, c\right)\right) d a_{1} \ldots d b_{\mathrm{g}} d_{\Theta} c \\
= & \operatorname{vol}(G) \int_{U \times V} H(\Phi(u, v)) Q_{t}(u) \frac{\left|\operatorname{det} D_{2} \Phi_{(u, v)}\right|}{\left|\operatorname{det} d \Pi_{\Phi(u, v)}^{*}\right|} d u d \sigma(v),
\end{aligned}
$$

where $d u$ is the (restriction to $U$ of the) usual unit-mass Haar measure on $G, d \sigma(v)$ the Riemannian volume measure on $V \subset \Pi^{-1}(e)$, and vol denotes volume measured 
with respect to the Riemannian metrics on $G$ and $\Theta$ induced by $\langle\cdot, \cdot\rangle_{g}$. Since $H$ is continuous and has compact support contained in $W$, we can apply the heat-kernel property $\lim _{t \rightarrow 0} \int_{G} h(x) Q_{t}(x) d x=h(e)$ valid for all continuous functions $h$ on $G$, to conclude that

$$
\begin{aligned}
& \lim _{t \downarrow 0} \int_{G^{28} \times \Theta} H\left(a_{1}, \ldots, b_{\mathrm{g}}, c\right) Q_{t}\left(\Pi\left(a_{1}, \ldots, b_{\mathrm{g}}, c\right)\right) d a_{1} \ldots d b_{\mathrm{g}} d_{\Theta} c \\
& =\operatorname{vol}(G)^{1-2 \mathrm{~g}} \operatorname{vol}(\Theta)^{-1} \int_{V} H(\Phi(e, v)) \frac{\left|\operatorname{det} D_{2} \Phi_{(e, v)}\right|}{\left|\operatorname{det} d \Pi_{\Phi(e, v)}^{*}\right|} d \sigma(v) .
\end{aligned}
$$

Since $\left|\operatorname{det} D_{2} \Phi_{(e, v)}\right|$ is the Jacobian, at $v$, of the map $V \rightarrow V: v \mapsto \Phi(e, v)$, we have

$$
\begin{aligned}
& \lim _{t \downarrow 0} \int_{G^{28} \times \Theta} H\left(a_{1}, \ldots, b_{\mathrm{g}}, c\right) Q_{t}\left(\Pi\left(a_{1}, \ldots, b_{\mathrm{g}}, c\right)\right) d a_{1} \ldots d b_{\mathrm{g}} d_{\Theta} c \\
& =\operatorname{vol}(G)^{1-2 \mathrm{~g}_{\operatorname{vol}}(\Theta)^{-1} \int_{V} H(v)} \frac{1}{\left|\operatorname{det} d \Pi_{v}^{*}\right|} d \sigma(v) \\
& =\operatorname{vol}(G)^{1-2 \mathrm{~g}} \operatorname{vol}(\Theta)^{-1} \int_{\Pi^{-1}(e)} H(v) \frac{1}{\left|\operatorname{det} d \Pi_{v}^{*}\right|} d \sigma(v),
\end{aligned}
$$

where in the last line we used again the fact that $H$ is supported in $W$. By a standard partition-of-unity argument, we conclude that

$$
\begin{aligned}
& \lim _{t \downarrow 0} \int_{G^{28} \times \Theta} H\left(a_{1}, \ldots, b_{\mathrm{g}}, c\right) Q_{t}\left(\Pi\left(a_{1}, \ldots, b_{\mathrm{g}}, c\right)\right) d a_{1} \ldots d b_{\mathrm{g}} d_{\Theta} c \\
& =\operatorname{vol}(G)^{1-2 \mathrm{~g}} \operatorname{vol}(\Theta)^{-1} \int_{\Pi^{-1}(e)} H(v) \frac{1}{\left|\operatorname{det} d \Pi_{v}^{*}\right|} d \sigma(v)
\end{aligned}
$$

holds for every continuous function $H$ on $G^{2 \mathrm{~g}} \times \Theta$.

Applying this to the limiting formulas (8.2.1) and (8.2.2) we have

$$
\begin{aligned}
& \lim _{T \downarrow 0} \int f\left(h\left(\kappa_{1} ; \omega\right), \ldots, h\left(\kappa_{n} ; \omega\right)\right) d \mu_{T}^{\Theta}(\omega) \\
& =N_{0}(\Theta)^{-1} \operatorname{vol}(G)^{1-2 \mathrm{~g}} \operatorname{vol}(\Theta)^{-1} \\
& \int_{\Pi^{-1}(e)} F(e, \ldots, e, \alpha, c)\left|\operatorname{det}\left(d \Pi_{(\alpha, c)}\right)^{*}\right|^{-1} d \sigma(\alpha, c)
\end{aligned}
$$

with

$$
N_{0}(\Theta)=\operatorname{vol}(G)^{1-2 \mathrm{~g}} \operatorname{vol}(\Theta)^{-1} \int_{\Pi^{-1}(e)}\left|\operatorname{det}\left(d \Pi_{(\alpha, c)}\right)^{*}\right|^{-1} d \sigma(\alpha, c)
$$

and $d \sigma$ being the Riemannian volume-measure on $\Pi^{-1}(e)$; the limiting formula (8.3.7), and the existence of the right side of (8.3.7), is contingent upon $N_{0}(\theta)$ being positive and finite. Positivity of $N_{0}(\theta)$ is clear from (8.3.8) since $\Pi^{-1}(e) \neq \emptyset$; finiteness will be shown below in (8.3.13).

Let $\Pi^{-1}(e)^{0}$ be the dense open subset of $\Pi^{-1}(e)$ on which the isotropy of the $G$ action on the manifold is $Z(G)$. Then by standard facts from the theory of transformation groups (Sect. 16.4.1(i), Problem 16.10.1 and Problem 12.10.1(a) in [Die]), the projection $\Pi^{-1}(e)^{0} \rightarrow \mathcal{M}(\Theta)^{0}$ is a principal $G / Z(G)$-bundle over the manifold $\mathcal{M}(\Theta)^{0}$. Since $f$ is $G$-invariant, it follows that so is $F$ and hence the function $F(e, \ldots, e,(\cdot)) \mid \Pi^{-1}(e)^{0}$ 
is constant on the fibers; we will sometimes view $F(e, \ldots, e,(\cdot))$ as a function on the quotient $\Pi^{-1}(e)^{0} / G$. Writing $F(e, \ldots, e,(\cdot)) \mid \Pi^{-1}(e)^{0}$ as a sum of functions supported on subsets of $\Pi^{-1}(e)^{0}$ on which there are local-trivializations, it follows from (8.3.7) (see [KS1] or Lemma 5.8 of [Se5] for details of this argument) that

$$
\begin{aligned}
& \lim _{T \downarrow 0} \int f\left(h\left(\kappa_{1} ; \omega\right), \ldots, h\left(\kappa_{n} ; \omega\right)\right) d \mu_{T}^{\Theta}(\omega) \\
& \quad=N_{0}(\Theta)^{-1} \frac{\operatorname{vol}(G)^{1-2 g}}{\operatorname{vol}(\Theta)} \operatorname{vol}(G / Z(G)) \\
& \int_{\Pi^{-1}(e)^{0} / G} F(e, \ldots, e, \alpha, c) \frac{\left|\operatorname{det} \gamma_{(\alpha, c)}^{\prime}\right|}{\left|\operatorname{det}\left(d \Pi_{(\alpha, c)}\right)^{*}\right|} d \bar{\sigma}(\alpha, c)
\end{aligned}
$$

and

$$
N_{0}(\Theta)=\frac{\operatorname{vol}(G)^{2-2 g}}{\operatorname{vol}(\Theta) \cdot \# Z(G)} \int_{\Pi^{-1}(e)^{0} / G} \frac{\left|\operatorname{det} \gamma_{(\alpha, c)}^{\prime}\right|}{\left|\operatorname{det}\left(d \Pi_{(\alpha, c)}\right)^{*}\right|} d \bar{\sigma}(\alpha, c),
$$

with $d \bar{\sigma}$ being the volume-measure on $\Pi^{-1}(e)^{0} / G$ corresponding to the Riemannian structure on $\Pi^{-1}(e)^{0} / G$ (this being as in (7.4.4)). Recall from Lemma 7.8 that

$$
\frac{\left|\operatorname{det} \gamma_{(\alpha, c)}^{\prime}\right|}{\left|\operatorname{det} d \Pi_{(\alpha, c)}^{*}\right|}=\left|\operatorname{Pf}\left(\bar{\Omega}_{\Theta}\right)\right|\left|\operatorname{det}(1-\operatorname{Ad} c)^{-1}\right|^{-1 / 2}
$$

where the Pfaffian $\left|\operatorname{Pf}\left(\bar{\Omega}_{\Theta}\right)\right|$ is the square-root of $\left|\operatorname{det} \bar{\Omega}_{\Theta}\right|$. Furthermore, since $\operatorname{Pf}\left(\bar{\Omega}_{\Theta}\right) d \bar{\sigma}=d \operatorname{vol}_{\bar{\Omega}_{\Theta}}$, we see that the right side of $(8.3 .9)$ is given by

$$
\begin{gathered}
N_{0}(\Theta)^{-1}\left|\operatorname{det}(1-\operatorname{Ad} c)^{-1}\right|^{-1 / 2} \frac{\operatorname{vol}(G)^{1-2 g}}{\operatorname{vol}(\Theta)} \operatorname{vol}(G / Z(G)) \\
\cdot \int_{\Pi^{-1}(e)^{0} / G} F(e, \ldots, e, \alpha, c) d \operatorname{vol}_{\bar{\Omega}_{\Theta}} \overline{(\alpha, c)}
\end{gathered}
$$

and, recalling the identifications $\Pi^{-1}(e) / G \simeq \mathcal{M}(\Theta)$ and $\Pi^{-1}(e)^{0} / G \simeq \mathcal{M}(\Theta)^{0}$,

$$
N_{0}(\Theta)=\left|\operatorname{det}(1-\operatorname{Ad} c)^{-1}\right|^{-1 / 2} \frac{\operatorname{vol}(G)^{2-2 g}}{\operatorname{vol}(\Theta) \cdot \# Z(G)} \operatorname{vol}_{\bar{\Omega}_{\theta}}\left(\mathcal{M}(\Theta)^{0}\right)
$$

where $c$ is any point in $\Theta$. The hypothesis that vol $\bar{\Omega}_{\theta}\left(\mathcal{M}(\Theta)^{0}\right)$ is finite now shows that $N_{0}(\Theta)$ is finite and this justifies (8.3.7) and hence also (8.3.9) and (8.3.12).

Now returning to the relationship between $f$ and $F$ explained in (8.1.3), (8.1.5) and in the remarks following (8.1.5), we have

$$
f\left(h\left(\kappa_{1} ; \omega\right), \ldots, h\left(\kappa_{n} ; \omega\right)\right)=F\left(e, \ldots, e, a_{1}, \ldots, b_{\mathrm{g}}, c\right)
$$

for any flat connection $\omega$ with $h\left(A_{i} ; \omega\right)=a_{i}, h\left(B_{i} ; \omega\right)=b_{i}$ and $h(C ; \omega)=c$. Combining (8.3.9), (8.3.12) and (8.3.14), the proof is complete. 


\section{The Case of $S U(2)$ Bundles}

In this section we specialize the considerations of the preceding Sects. to the case $G=S U(2)$ and show that a sharp form of the semiclassical limit formula holds. We also determine the symplectic volume of $\mathcal{M}(\theta)$ explicitly.

We shall exclude the case where $\Theta$ consists of one point (which must be one of the matrices $\pm I)$. The excluded cases are essentially contained in the theory for $S U(2)$ and $S O(3)$ flat connections over closed surfaces, and this theory is treated fully in [Se5].

\section{1 .}

Theorem 7. Let the group $G$ be $S U(2)$, and let $\Theta$ be a conjugacy class containing more than one point (i.e. $\Theta$ is not the conjugacy class of $I$ or of $-I$ ). Then:

[i] $\mathcal{M}(\Theta)$ is a smooth $(6 \mathrm{~g}-4)$ - dimensional manifold which is connected.

[ii] the $2-$ form $\bar{\Omega}_{\Theta}$ on $\mathcal{M}(\Theta)$ is symplectic and the corresponding volume of $\mathcal{M}(\Theta)$ is

$$
\operatorname{vol}_{\bar{\Omega}_{\Theta}}(\mathcal{M}(\Theta))=\left\{\begin{array}{ll}
2 \pi\left(\pi-\theta_{c}\right)\left[\frac{\operatorname{vol}_{(S U(2))}}{2 \pi^{2}}\right]^{\frac{2}{3}} & \text { if } \mathrm{g}=1 \\
4 \pi \sin \theta_{c}\left[\frac{\operatorname{vol}(S U(2))}{2 \pi^{2}}\right]^{\frac{2}{3}} \operatorname{vol}(S U(2))^{2 \mathrm{~g}-2} \sum_{n=1}^{\infty} \frac{\chi_{n}(c)}{n^{2 g}-1} & \text { if } \mathrm{g} \geq 2
\end{array},\right.
$$

where $\chi_{n}$ is the character function of $S U(2)$ specified below in (9.1.7), $c$ is any point in the conjugacy class $\Theta, \theta_{c}$ is the number in $(0, \pi)$ for which $\cos \theta_{c}=\operatorname{Tr}(c) / 2$, and vol $(S U(2))$ is the volume of $S U(2)$ with respect to the fixed metric $\langle\cdot, \cdot\rangle_{g}$ on its Lie algebra.

[iii] if $\mathcal{S}$ be a triangulation of $\Sigma$, and $\kappa_{1}, \ldots, \kappa_{n}$ loops based at o made up of oriented 1 - simplices of $\mathcal{S}$ as in Sect. then for any continuous conjugation-invariant function $f$ on $G^{n}$,

$$
\begin{aligned}
& \lim _{T \downarrow 0} \int f\left(h\left(\kappa_{1} ; \omega\right), \ldots, h\left(\kappa_{n} ; \omega\right)\right) d \mu_{T}^{\Theta}(\omega) \\
& =\frac{1}{v o l_{\bar{\Omega}_{\Theta}}(\mathcal{M}(\Theta))} \int_{\mathcal{M}(\Theta)} f\left(h\left(\kappa_{1} ; \omega\right), \ldots, h\left(\kappa_{n} ; \omega\right)\right) d v o \bar{\Omega}_{\bar{\Omega}_{\Theta}}([\omega]) .
\end{aligned}
$$

Proof. (i) In SU(2) any element not in the center lies in a unique maximal torus, and two elements commute if and only if both lie in the same maximal torus. This implies that the isotropy of the $G$ conjugation action at any $\left(a_{1}, \ldots, b_{\mathrm{g}}, c\right) \in \Pi^{-1}(I) \subset G^{2 \mathrm{~g}} \times \Theta$ (with $I$ being the identity matrix, and $\Theta$ being other than the one-point conjugacy class $\{I\})$ is $Z(G)=\{ \pm I\}$. Consequently, from (7.4.1), $\Pi$ is a submersion at every point of $\Pi^{-1}(I)$, and so $\Pi^{-1}(I)$ is a smooth submanifold of $G^{2 \mathrm{~g}} \times \Theta$, of dimension $3(2 \mathrm{~g})+2-3=6 \mathrm{~g}-1$. Since the isotropy group of the $G$ action is $\{ \pm I\}$ everywhere it follows, by standard facts from transformation group theory alluded to earlier ([Die]), that $\Pi^{-1}(I) / G \simeq \mathcal{M}(\Theta)$ is a smooth manifold of dimension $6 \mathrm{~g}-4$, and $\Pi^{-1}(I) \rightarrow \Pi^{-1}(I) / G \simeq \mathcal{M}(\Theta)$ is a smooth principal $S U(2) /\{ \pm I\}$-bundle. Let $\left(\alpha_{1}, c_{1}\right),\left(\alpha_{2}, c_{2}\right) \in \Pi^{-1}(I)$; in particular, $c_{1}, c_{2} \in \Theta$. Since $G=S U(2)$ is connected, there is a continuous path $[0,1] \rightarrow G: t \mapsto$ $x_{t}$ such that $x_{0}=I$ and $x_{1} c_{1} x_{1}^{-1}=c_{2}$. Therefore $t \mapsto\left(x_{t} \alpha_{1} x_{t}^{-1}, x_{t} c_{1} x_{t}^{-1}\right)$ is a path in $\Pi^{-1}(I)$ from $\left(\alpha_{1}, c_{1}\right)$ to a point $\left(\alpha_{2}^{\prime}, c_{2}\right)$ where $\alpha_{2}^{\prime}$ lies in $K^{-1}\left(c_{2}^{-1}\right)$, where $K$ is the product commutator map

$$
K: G^{2 \mathrm{~g}} \rightarrow G:\left(a_{1}, b_{1}, \ldots, a_{\mathrm{g}}, b_{\mathrm{g}}\right) \mapsto b_{\mathrm{g}}^{-1} a_{\mathrm{g}}^{-1} b_{\mathrm{g}} a_{\mathrm{g}} \cdots b_{1}^{-1} a_{1}^{-1} b_{1} a_{1} .
$$


In Proposition 3.10 of [Se5], it was proven that the set $K^{-1}\left(c^{-1}\right)$ is connected for every $c \in S U(2)$. Consequently, $\alpha_{2}^{\prime}$ can be connected to $\alpha_{2}$ by a continuous path lying in $K^{-1}\left(c_{2}^{-1}\right)$. Combining all these observations we conclude that $\left(\alpha_{1}, c_{1}\right)$ can be connected to $\left(\alpha_{2}, c_{2}\right)$ by a continuous path in $\Pi^{-1}(I)$.

(ii) Recall from (8.3.13) that, since now $\mathcal{M}(\Theta)^{0}=\mathcal{M}(\Theta)$,

$$
\begin{aligned}
& \lim _{t \rightarrow 0} \int_{G^{2 \mathrm{~g}} \times \Theta} Q_{t}\left(c b_{\mathrm{g}}^{-1} a_{\mathrm{g}}^{-1} b_{\mathrm{g}} a_{\mathrm{g}} \cdots b_{1}^{-1} a_{1}^{-1} b_{1} a_{1}\right) d a_{1} \cdots d b_{\mathrm{g}} d_{\Theta} c \\
& =\left|\operatorname{det}(1-\operatorname{Ad} c)^{-1}\right|^{-1 / 2} \frac{\operatorname{vol}(G)^{2-2 \mathrm{~g}}}{\operatorname{vol}(\Theta) \cdot \# Z(G)} \operatorname{vol}_{\bar{\Omega}_{\Theta}}(\mathcal{M}(\Theta)) .
\end{aligned}
$$

By definition of $d_{\Theta} c, \int_{\Theta} h(c) d_{\Theta} c=\int_{G} h\left(x c_{1} x^{-1}\right) d x$ for any $c_{1} \in \Theta$. It follows then by conjugation invariance of $Q_{t}$ that for any $c \in \Theta$,

$$
\begin{aligned}
& \int_{G^{2 \mathrm{~g}} \times \Theta} Q_{t}\left(c b_{\mathrm{g}}^{-1} a_{\mathrm{g}}^{-1} b_{\mathrm{g}} a_{\mathrm{g}} \cdots b_{1}^{-1} a_{1}^{-1} b_{1} a_{1}\right) d a_{1} \cdots d b_{\mathrm{g}} d_{\Theta} c \\
& =\int_{G^{2 \mathrm{~g}}} Q_{t}\left(c b_{\mathrm{g}}^{-1} a_{\mathrm{g}}^{-1} b_{\mathrm{g}} a_{\mathrm{g}} \cdots b_{1}^{-1} a_{1}^{-1} b_{1} a_{1}\right) d a_{1} \cdots d b_{\mathrm{g}}
\end{aligned}
$$

The heat kernel $Q_{t}$ has a standard expansion in terms of characters of the group $G$. Using this, it is proven in Lemma 5.5 of [Se5] (see also [Wi1]) that

$$
\begin{aligned}
& \lim _{t \rightarrow 0} \int_{G^{2 \mathrm{~g}}} Q_{t}\left(c b_{\mathrm{g}}^{-1} a_{\mathrm{g}}^{-1} b_{\mathrm{g}} a_{\mathrm{g}} \cdots b_{1}^{-1} a_{1}^{-1} b_{1} a_{1}\right) d a_{1} \cdots d b_{\mathrm{g}} \\
& = \begin{cases}\frac{\pi-\theta_{c}}{2 \sin \theta_{c}} & \text { if } \mathrm{g}=1 \\
\sum_{n=1}^{\infty} \frac{\chi_{n}(c)}{n^{2 g-1}} & \text { if } \mathrm{g} \geq 2\end{cases}
\end{aligned}
$$

where

$$
\theta_{c} \in(0, \pi) \text { is defined by } \cos \theta_{c}=\operatorname{Tr}(c) / 2,
$$

i.e. $c$ is conjugate to $\left(\begin{array}{cc}e^{i \theta_{c}} & 0 \\ 0 & e^{-i \theta_{c}}\end{array}\right)$, and $\chi_{n}$ is the character of the $n$-dimensional irreducible representation of $S U(2)$ :

$$
\chi_{n}(c)=\frac{\sin \left[n \cos ^{-1}\left\{\frac{1}{2} \operatorname{Tr}(c)\right\}\right]}{\sin \left[\cos ^{-1}\left\{\frac{1}{2} \operatorname{Tr}(c)\right\}\right]} \text { for every } c \in S U(2) \backslash\{ \pm I\} .
$$

Combining this with (9.1.3) we have, with $\theta_{c}$ as in (9.1.6),

$$
\operatorname{vol}_{\bar{\Omega}_{\Theta}}(\mathcal{M}(\Theta))=\left\{\begin{array}{ll}
2\left|\operatorname{det}(1-\operatorname{Ad} c)^{-1}\right|^{\frac{1}{2}} \operatorname{vol}(\Theta) \frac{\pi-\theta_{c}}{2 \sin \theta_{c}} & \text { if } g=1 \\
2\left|\operatorname{det}(1-\operatorname{Ad} c)^{-1}\right|^{\frac{1}{2}} \operatorname{vol}(\Theta) \operatorname{vol}(G)^{2 g-2} \sum_{n=1}^{\infty} \frac{\chi_{n}(c)}{n^{2 g-1}} & \text { if } g \geq 2
\end{array} .\right.
$$

Next we compute $\operatorname{vol}(\Theta)$. Fix $c \in \Theta$. If we view $S U(2)$ as a 3 -sphere in $\mathbf{R}^{4}$ in the usual way, the angle between $c$ and $I$ is $\theta_{c}$. So the surface area of the 2-sphere $\Theta$ is

$$
\operatorname{vol}(\Theta)=4 \pi \sin ^{2} \theta_{c}\left[\frac{\operatorname{vol}(S U(2))}{2 \pi^{2}}\right]^{\frac{2}{3}}
$$


where of course the volume of $S U(2)$ is with respect to the metric $\langle\cdot, \cdot\rangle_{g}$ on the Lie algebra of $S U(2)$.

The mapping $e^{i \theta} \mapsto \operatorname{Ad}\left(\begin{array}{cc}e^{i \theta} & 0 \\ 0 & e^{-i \theta}\end{array}\right)$ is a homomorphism of the circle group onto the group of rotations in a plane in $g$ and has kernel $\{ \pm 1\}$; therefore Ad $c$ is rotation by angle $2 \theta_{c}$ in a plane orthogonal to the maximal torus of $c$, and so

$$
\left|\operatorname{det}(1-\operatorname{Ad} c)^{-1}\right|^{\frac{1}{2}}=\operatorname{det}\left[\begin{array}{cc}
1-\cos 2 \theta_{c} & \sin 2 \theta_{c} \\
-\sin 2 \theta_{c} & 1-\cos 2 \theta_{c}
\end{array}\right]^{-\frac{1}{2}}=\frac{1}{2 \sin \theta_{c}}
$$

Substituting this into (9.1.8) yields the volume formula (9.1.1).

(iii) is a special case of Theorem 8.3, bearing in mind that now $\mathcal{M}(\Theta)^{0}=\mathcal{M}(\Theta)$, and that, by (ii), this has finite volume.

\subsection{Remarks.}

(i) As already noted,the cases $\Theta=\{I\}$ and $\Theta=\{-I\}$ are essentially contained in the study of the moduli space of flat connections over the trivial $S U(2)$-bundle and the non-trivial $S O(3)$-bundle over the closed surface of genus $\mathrm{g}$ dealt with in [Se4,5]. The following observations are based on [Se4,5]. The moduli space $\mathcal{M}(I)$ consists of a number of strata whose structure and volumes have been determined in [Se4,5]. The moduli space $\mathcal{M}(-I)$ consists of one point if $\mathrm{g}=1$; if $\mathrm{g} \geq 2$ then $\mathcal{M}(-I)$ is a smooth connected $3(2 \mathrm{~g}-2)-$ dimensional manifold. The $2-$ form $\bar{\Omega}_{\Theta}$ is symplectic and the corresponding volume is obtained by considering

$$
\lim _{t \rightarrow 0+} \int_{G^{2 g}} Q_{t}\left(-I \cdot b_{\mathrm{g}}^{-1} a_{\mathrm{g}}^{-1} b_{\mathrm{g}} a_{\mathrm{g}} \cdots b_{1}^{-1} a_{1}^{-1} b_{1} a_{1}\right) d a_{1} \cdots d b_{\mathrm{g}} ;
$$

this leads to the value $2 \cdot \operatorname{vol}(S U(2))^{2 g-2} \sum_{n=1}^{\infty}(-1)^{n-1} / n^{2 g-2}$ (which is the same as is obtained if we set $\operatorname{vol}(\Theta)$ and the determinant factor equal to 1 in the formula (9.1.8)).

(ii) If in the first formula in (9.1.1) (the case $\mathrm{g}=1$ ) we set $\theta_{c}=0$, we obtain the value $2 \pi^{2}\left[\frac{\operatorname{vol}(S U(2))}{2 \pi^{2}}\right]^{\frac{2}{3}}$ which is exactly the volume computed in the closed-surface theory of [Se5] (and in Lemma 3.11 in [Se4]). Such a comparison cannot be made for $\mathrm{g} \geq 2$ since in this case the dimension of $\mathcal{M}(\Theta)$ collapses at $\Theta=\{I\}$.

(iii) If we set $g=1$ in the second formula (i.e. the one for $g \geq 2$ ) in (9.1.1) and use the trigonometric sum formula $\sum_{n=1}^{\infty}(\sin n \theta) / n=(\pi-\theta) / 2$ for $\theta \in(0, \pi)$, then what results is the first formula in (9.1.1) (i.e. the one for $g=1$ ); thus the second formula actually covers all cases.

\section{Appendix}

We will quote some results from [Se2,3] and indicate briefly how they are applied in Sects. 3 and 5 to the construction of $\mu_{T}^{c}$ and $\mu_{T}^{\Theta}$.

The basic conditional proability result we need is (from [Se2]):

A1. Theorem. Let $\left(\Omega_{i}, \mathcal{F}_{i}, P_{i}\right)$, for $i=1,2$ be probability spaces, where $\Omega_{1}$ and $\Omega_{2}$ are complete separable metric spaces and the corresponding $\mathcal{F}_{i}$ are the Borel $\sigma$-algebras. 
Let $G$ be a compact Lie group. Consider random variables $h_{i}: \Omega_{i} \rightarrow G$, for $i=1,2$, each having positive density with respect to Haar measure on $G$. Then there is a unique assignment

$$
\left(\mathcal{F}_{1} \otimes \mathcal{F}_{2}\right) \times G \rightarrow[0,1]:(E, z) \mapsto P\left(E \mid h_{1} h_{2}=z\right)
$$

such that the following hold:

(i) $E \mapsto P\left(E \mid h_{1} h_{2}=z\right)$ is a probability measure for every $z \in G$.

(ii) $z \mapsto P\left(E \mid h_{1} h_{2}=z\right)$ is measurable for every $E \in \mathcal{F}_{1} \otimes \mathcal{F}_{2}$, and

$$
\int_{V} P\left(E \mid h_{1} h_{2}=z\right) d p_{12}(z)=\left(P_{1} \otimes P_{2}\right)\left(E \cap\left\{h_{1} h_{2} \in V\right\}\right)
$$

holds for every Borel $V \subset G$, where $p_{12}$ is the probability measure on $G$ describing the distribution of $h_{1} h_{2}$

(iii) $z \mapsto P\left(A \times B \mid h_{1} h_{2}=z\right)$ is continuous for every $A \in \mathcal{F}_{1}$ and $B \in \mathcal{F}_{2}$. Moreover,

$$
P\left(\left\{h_{1} h_{2}=z\right\} \mid h_{1} h_{2}=z\right)=1
$$

Using the conditional measure described above, we can construct another conditional measure, which is the one we use for the measures $\mu_{T}^{c}$ and $\mu_{T}^{\Theta}$.

A2. Definition. Let $\left(\Omega_{i}, \mathcal{F}_{i}, P_{i}\right)$, for $i=1,2,3$, be probability spaces, with $\left(\Omega_{1}, \mathcal{F}_{1}, P_{1}\right)$ and $\left(\Omega_{2}, \mathcal{F}_{2}, P_{2}\right)$ being as in Theorem A1. Let $G$ be a compact Lie group and consider $G$-valued random variables $h_{i}$ on $\Omega_{i}$, with $h_{1}$ and $h_{2}$ having positive densities with respect to Haar measure on $G$. Consider the product space $(\Omega, \mathcal{F})=\left(\Omega_{1}, \mathcal{F}_{1}\right) \times$ $\left(\Omega_{2}, \mathcal{F}_{2}\right) \times\left(\Omega_{3}, \mathcal{F}_{3}\right)$; for $E \in \mathcal{F}$ we define

$$
P\left(E \mid h_{1} h_{2}=h_{3}\right) \stackrel{\text { def }}{=} \frac{1}{Z} \int_{\Omega_{3}} d P_{3}\left(\omega_{3}\right) P\left(E^{\omega_{3}} \mid h_{1} h_{2}=h_{3}\left(\omega_{3}\right)\right) \rho_{12}\left(h_{3}\left(\omega_{3}\right)\right)
$$

where $\rho_{12}$ is the density of $h_{1} h_{2}$ with respect to Haar measure on $G$, and $E^{\omega_{3}}=$ $\left\{\left(\omega_{1}, \omega_{2}\right):\left(\omega_{1}, \omega_{2}, \omega_{3}\right) \in E\right\}$, and

$$
Z=\int_{\Omega_{3}} \rho_{12}\left(h_{3}\left(\omega_{3}\right)\right) d P_{3}\left(\omega_{3}\right),
$$

Then $P\left(\cdot \mid h_{1} h_{2}=h_{3}\right)$ makes sense, is a probability measure on $(\Omega, \mathcal{F})$, and satisfies

$$
P\left(\left\{h_{1} h_{2}=h_{3}\right\} \mid h_{1} h_{2}=h_{3}\right)=1 .
$$

Thus $P\left(\cdot \mid h_{1} h_{2}=h_{3}\right)$ really lives on the subset of $\Omega$ where $h_{1} h_{2}=h_{3}$.

A3. Construction of $\mu_{\mathrm{T}}^{\mathbf{c}}$. Fix a positive real number $T$. We shall describe the details of the construction of $\mu_{T}^{c}$, for any $c \in G$, in terms of the conditional probability measure described in Definition A2. For the sake of notational simplicity we shall only describe the case considered in Sect. 3, i.e. of the torus with one hole. The general case is exactly analogous and is discussed briefly in Sect. A5 below.

For the construction of $\mu_{T}^{c}$, divide the disk $D$ into an upper half $D_{U}$ and a lower half $D_{L}$. The boundaries $\partial D_{L}$ and $\partial D_{U}$, when considered as loops, will be taken to start from the center $O$ of the disk $D$. Thus, with $L_{0}$ being the radial path from $O$ to $x_{0}=(1,0) \in \partial D$, we have

$$
h\left(\partial D_{L} ; \omega\right) h\left(\partial D_{U} ; \omega\right)=h\left(\bar{L}_{0} \cdot \partial D \cdot L_{0} ; \omega\right)
$$


for any connection $\omega$ over $D$.

Equip $D$ with the measure which corresponds, via the quotient map $q: D \rightarrow \Sigma$, to the Riemannian area measure on $\Sigma$. For any subset $A \subset D$ (such as $A=D_{U}$ or $\left.A=D_{L}\right)$ let $L^{2}(A ; g)$ be the corresponding Hilbert space of $g$-valued square-integrable functions on $D$ vanishing outside $A$.

Let $\Omega_{1}$ be a Hilbert-Schmidt closure of $L^{2}\left(D_{U} ; g\right)$ and $P_{1}^{T}$, or simply $P_{1}$, the standard Gaussian measure, with variance scaled by $T$, on the Borel $\sigma$-algebra $\mathcal{F}_{1}$ of $\Omega_{1}$ :

thus for each $f \in L^{2}\left(D_{U} ; g\right)$ there is a Gaussian random variable $\tilde{f}$ on $\Omega_{1}$, such that for any $f_{1}, f_{2} \in L^{2}\left(D_{U} ; \underline{g}\right),\left\langle\tilde{f}_{1}, \tilde{f}_{2}\right\rangle_{L^{2}\left(P_{1}\right)}=T\left\langle f_{1}, f_{2}\right\rangle_{L^{2}\left(D_{U} ; \underline{g}\right)}$.

The $g$-valued white-noise referred to in Sect. 3.2 (or at least the part of it over $D_{U}$ ) is obtained, for instance, by choosing any orthonormal basis $e_{1}, \ldots, e_{d}$ of $\underline{g}$ and setting $F^{\omega}(E)=\sum_{i=1}^{d}\left(1_{E} e_{i}\right) e_{i}$ for every Borel $E \subset D_{U}$.

Let $\left(\Omega_{2}, \mathcal{F}_{2}, P_{2}\right)$ be the corresponding space of $D_{L}$ (we are suppressing the superscript $T$ in $P_{2}^{T}$ ).

We modify the definition of $\Omega_{\text {disk }}$ given in Sect. 3.2 to:

$$
\Omega_{\mathrm{disk}}=\Omega_{U} \times \Omega_{L} .
$$

Finally, let $\Omega_{3}=G^{2}$, and let $P_{3}$ be the unit-mass Haar measure on $G^{2}$. The functions $h_{i}$ are as follows:

$$
\begin{aligned}
& h_{1}\left(\omega_{1}\right)=h\left(\partial D_{U} ; \omega_{1}\right), \\
& h_{2}\left(\omega_{2}\right)=h\left(\partial D_{L} ; \omega_{2}\right), \\
& h_{3}(a, b)=c b^{-1} a^{-1} b a,
\end{aligned}
$$

In (A3.4) and (A3.5), the left sides are defined in terms of solutions of the stochastic differential equation (3.2.1) and, as pointed out after (3.2.1), are $G$-valued random variables with densities $Q_{T\left|D_{U}\right|}(\cdot)$ and $Q_{T\left|D_{L}\right|}(\cdot)$, respectively.

As in Sect. 3.3, we define

$$
\Omega_{c}=\Omega_{\mathrm{disk}} \times G^{2},
$$

and we define the measure $\mu_{T}^{c}$ by

$$
\mu_{T}^{c}=P\left(\cdot \mid h_{1} h_{2}=h_{3}\right),
$$

where the right side is the probability measure specified above in Definition A2.

Although $\Omega_{c}$ does not depend on $c$, it follows from $(A 2.3)$ that the measure $\mu_{c}$ really lives on the subspace of $\omega$ for which the constraint

$$
h\left(\bar{L}_{0} \cdot \partial D \cdot L_{0} ; \omega\right)=c b^{-1} a^{-1} b a
$$

holds (the left side is defined to be the product $h\left(\partial D_{L} ; \omega_{2}\right) h\left(\partial D_{U} ; \omega_{1}\right)$, following (A3.1)).

From the expression in $(A 2.2)$ for the 'normalizer' $Z=Z_{T}(c)$, and from the observations made above concering the densities of $h_{1}$ and $h_{2}$, we have:

$$
Z_{T}(c)=\int_{G^{2}} Q_{T|\Sigma|}\left(c b^{-1} a^{-1} b a\right) d a d b
$$

which is the same as our earlier value (3.4.2). 
A4. Construction of $\mu_{T}^{\ominus}$. The construction of $\mu_{T}^{\Theta}$, where $\Theta$ is any conjugacy class in $G$, is similar to that for $\mu_{T}^{c}$. The only difference is that now we set

$$
\Omega_{3}=G^{2} \times \Theta
$$

take the measure $P_{3}$ to be (unit-mass Haar on $\left.G^{2}\right) \times($ the $G$-invariant unit-mass measure on $\Theta$ ), take $F_{3}$ to be given by $h_{3}(a, b, c)=c b^{-1} a^{-1} b a$, and define

$$
\Omega_{\Theta}=\Omega_{\text {disk }} \times G^{2} \times \Theta .
$$

The spaces $\left(\Omega_{i}, \mathcal{F}_{i}, P_{i}\right)$, for $i=1,2$, and the corresponding variables $h_{1}$ and $h_{2}$ are as for $\mu_{T}^{c}$. With this, we define

$$
\mu_{T}^{\Theta} \stackrel{\text { def }}{=} P\left(\cdot \mid h_{1} h_{2}=h_{3}\right) .
$$

The corresponding normalizer $Z=N_{T}(\Theta)$ is then:

$$
N_{T}(\Theta)=\int_{G^{2} \times \Theta} Q_{T|\Sigma|}\left(c b^{-1} a^{-1} b a\right) d a d b d_{\Theta} c
$$

which agrees with the value in (3.4.5).

A5. Other surfaces. For general compact surfaces (as described in Sect. 5), $\Omega_{3}$ is taken to be $G^{\sigma \mathrm{g}} \times G^{p}$ (with notation as in Sect. 5; $\sigma$ is 1 if $\Sigma$ is unorientable, 2 otherwise), and $h_{3}$ is taken to be the appropriate function of the $a_{i}, b_{i}, c_{i}$ given by the right side of (5.3.6).

Finally, we quote from (Proposition 4.5 of) [Se2] the exact expectation-value formula which was alluded to in Sect. 3.4.

A6. Proposition. With notation and hypotheses as in Sects. A2 and A3, let $\phi=$ $\left(\phi_{1}, \ldots, \phi_{m}\right): \Omega_{1} \rightarrow G^{m}$ and $\psi=\left(\psi_{1}, \ldots, \psi_{n}\right): \Omega_{2} \rightarrow G^{n}$ be measurable functions. Suppose that $\phi_{k} \cdots \phi_{1}=h_{1}$ and $\psi_{l} \cdots \psi_{1}=h_{2}$, where the $h_{i}$ are as in Sect. Al. Suppose also that $\phi$ has a bounded density $\rho_{\phi}$ on $G^{m}$, and $\psi$ has a bounded density $\rho_{\psi}$ on $G^{n}$. Then for any bounded measurable function $f$ on $G^{m} \times G^{n} \times \Omega_{3}$, we have (with $\left.\omega=\left(\omega_{1}, \omega_{2}, \omega_{3}\right)\right)$ :

$$
\begin{aligned}
& \int f\left(\phi\left(\omega_{1}\right), \psi\left(\omega_{2}\right), \omega_{3}\right) d P\left(\omega \mid h_{1} h_{2}=h_{3}\right) \\
& =\frac{1}{Z} \int f\left(x, y, \omega_{3}\right) \rho_{\phi}(x) \rho_{\psi}(y) \delta\left(x_{k} \cdots x_{1} y_{l} \cdots y_{l} h_{3}\left(\omega_{3}\right)^{-1}\right) d P_{3}\left(\omega_{3}\right),
\end{aligned}
$$

where $\delta(\cdot)$ means that we can drop any $x_{j}$ (with $1 \leq j \leq k$ ) or $y_{j}$ (with $1 \leq j \leq$ l) from the integration and replace it in the integrand with the value which makes $x_{k} \cdots x_{1} y_{l} \cdots y_{1}=h_{3}\left(\omega_{3}\right)$, and $Z$ is the normalizing constant given in $(A 2.2)$.

In applications, we work typically with random variables of the form $h(\kappa ; \omega)$, where $\kappa$ is an admissible loop in $D$ based at $O$; breaking $\kappa$ into pieces in $D_{L}$ and pieces in $D_{U}$ (and adjoining appropriate additional radial segments) we can express $h(\kappa ; \omega)$ as a product of variables $h\left(\kappa^{\prime} ; \omega\right)$, where $\kappa^{\prime}$ is a loop in $D_{L}$ or in $D_{U}$. Thus Proposition A6 is applicable in situations, where $\phi_{i}$ and $\psi_{i}$ are holonomy variables $h\left(\kappa^{\prime} ; \cdot\right)$.

Acknowledgement. At various stages of this work the author has received support from NSF grant DMS 9400961, U.S. Army Research Office \#DAAH04-94-G-0249, and from the Alexander von Humboldt Foundation. This paper was written in large part during my stay at the Institut für Mathematik, Ruhr-Universität Bochum, and it is a pleasure to thank Professor S. Albeverio and colleagues for their hospitality. 


\section{References}

[AB] Atiyah, M. and Bott, R.: The Yang-Mills Equations over Riemann Surfaces: Phil. Trans. R. Soc. Lond. A 308, 523-615(1982)

[AIK] Albeverio, S., Iwata, K. and Kolsrud, T.: Random Parallel Transport on Surfaces of Finite Type, and Relations to Homotopy. In: "Probabilistic Methods in Mathematical Physics". Eds. F. Guerra, M.T. Loffredo and C. Marchioro, Singapore, World Scientific, (1992)

[Be] C. Becker, Wilson Loops in Two-Dimensional Space-Time Regarded as White Noise, J. Funct. Anal. 134, 321-349(1995)

[BG] Biswas,I. and Guruprasad, K.: Principal bundles on open surfaces and invariant functions on Lie groups. Internat. J. Math. 4, 535-544(1993)

[Br] Brown, E. M.: The Hauptvermutung for 3-complexes, Trans. Am. Math. Soc. 144, 173-196 (1969)

[Bre] Bredon, G.: Introduction to Compact Transformation Groups, New York: Academic Press 1972

[Di] Dimock, J.: Canonical quantization of Yang-Mills on a circle. To appear in Rev. in Math. Phys.

[Die] Dieudonné, J.: Treatise on Analysis III. Transl. by I. G.Macdonald, New York: Academic Press 1972

[Fi] Fine, D.: Quantum Yang-Mills on Compact Surfaces. Commun. Math. Phys. 140, 321-338 (1991)

[Fo] Forman, R.: Small volume limits of 2-d Yang-Mills. Commun. Math. Phys. 151, 39-52 (1993)

[Go] Goldman, W.: The Symplectic Nature of Fundamental Groups of Surfaces, Adv. Math. 54, 200-225 (1984)

[Je] Jeffrey, L.C.: Extended moduli spaces of flat connections on Riemann surfaces. Math. Annalen 298, 667-692(1994)

[KS1] King, C. and Sengupta, A.: The Semiclassical Limit of the Two Dimensional Quantum Yang-Mills Model, J. Math. Phys. Special Issue on Topology and Physics, 10, 5354-5361 (1994)

[KS2] King, C. and Sengupta, A.: A new 2-form for connections on surfaces with boundary. Lett. Math. Phys. 34, 135-147 (1995)

[KS3] King, C. and Sengupta, A.: A symplectic structure for connections on surfaces with boundary. Commun. Math. Phys. 175, 657-671 (1996)

[RR] Rajeev S. G. and Rossi, L.: Some rigorous results for Yang-Mills theories on a cylinder. J. Math. Phys. Lett. 36, (1995)

[Se1] Sengupta A.: The Semiclassical Limit for Gauge Theory on $\mathrm{S}^{2}$. Commun. Math. Phys. 147, 191-197 (1992)

[Se2] Sengupta, A: Quantum Gauge Theory on Compact Surfaces. Ann. Phys. (NY)221, 17-52 (1993)

[Se3] Sengupta, A.: Gauge Theory on Compact Surfaces. To appear in Mem. of the Am. Math. Soc.

[Se4] Sengupta, A.: The Semiclassical Limit of $S U(2)$ and $S O(3)$ Gauge Theory on the Torus. Commun. Math. Phys. 169, 297-314 (1995)

[Se5] Sengupta, A.: The Semiclassical Limit of SU(2) and SO(3) Gauge Theory on Compact Oriented Surfaces. Preprint 1995

[Se6] Sengupta, A.: The Moduli Space of Yang-Mills Connections over a Compact Surface. To appear in Rev. Math. Phys. (1997)

[Wil] Witten, E.: On Quantum Gauge Theories in Two Dimensions,Commun. Math. Phys. 141, 153-209 (1991)

[Wi2] Witten, E.: Two Dimensional Quantum Gauge Theory revisited. J. Geom. Phys. 9, 303-368 (1992)

[Wi3] Witten, E.: Some Questions for Constructive Field Theorists. In "Constructive Physics: Results in Field Theory, Statistical Mechanics and Condensed Matter Physics'. Vincent Rivasseau (Ed.), Springer Berlin-Heidelberg-New York, pp.1-5 (1995)

Communicated by R. H. Dijkgraaf 
